\title{
A dual workflow to improve the proteomic coverage in plasma using data-independent acquisition-MS
}

\section{SUPPORTING INFORMATION FOR PUBLICATION}

Shenyan Zhang1,2, Koen Raedscheldersı, Vidya Venkatramanı, Lilith Huangı, Ronald

Holewinskil, Qin Ful, Jennifer E. Van Eyk ${ }^{*}$

1Advanced Clinical Biosystems Research Institute, Barbara Streisand Women's Heart Center of

The Smidt Heart Center, Cedars-Sinai Medical Center.

2BGI Genomics, Shenzhen, China.

Corresponding author: Jennifer E. Van Eyk (Jennifer.VanEyk@cshs.org)

\section{Contents}

Supplementary Figure 1

Supplementary Figure 2

Supplementary Table 1

Supplementary Table 2

Supplementary Table 3

Supplementary Table 4 


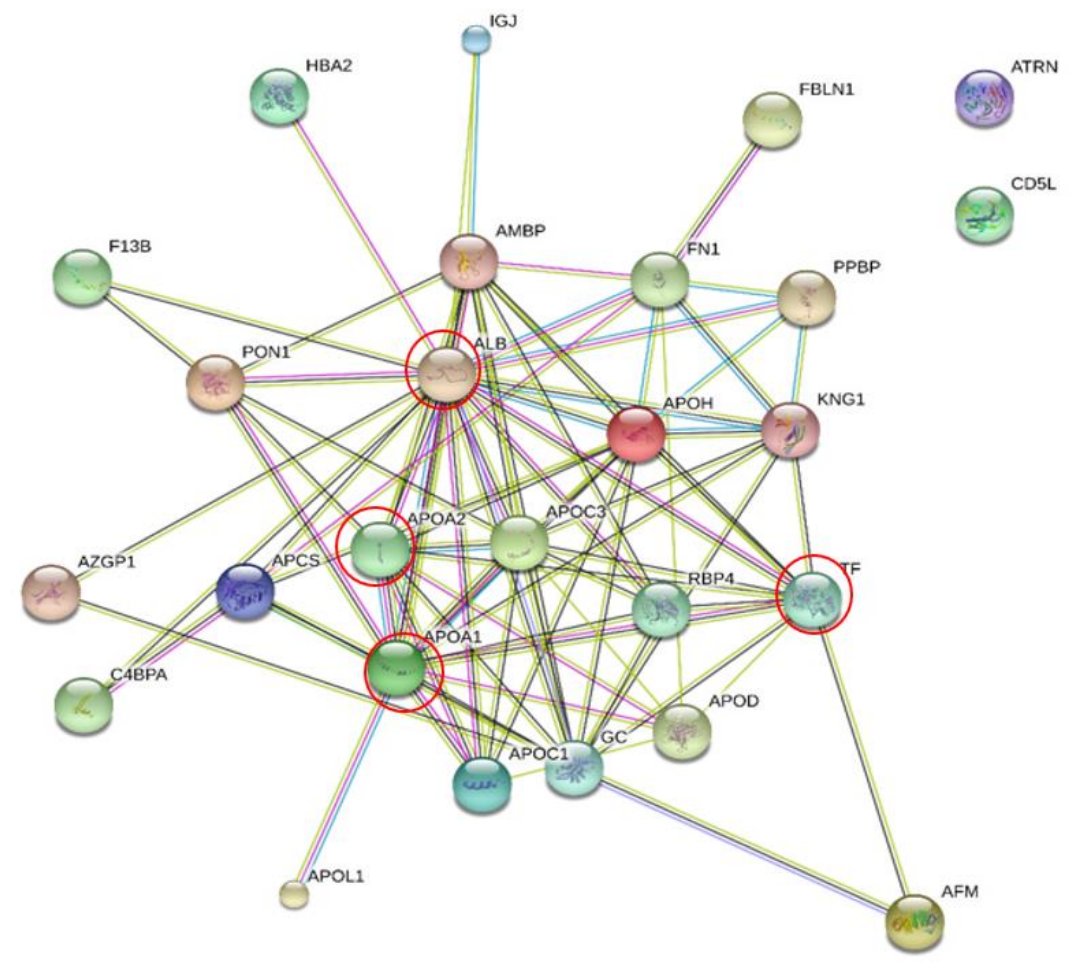

Nodes:

Network nodes represent proteins

splice isoforms or post-translational modifications are collapsed, i.e. each node represents all the

proteins produced by a single, protein-coding gene locus.

\section{Edges:}

Edges represent protein-protein associations associations are meant to be specific and meaningful, i.e. proteins jointly contribute to a shared function; this does not necessarily mean they are physically binding each other.
Node Size

e small nodes:

protein of unknown 3D structure

(28) large nodes:

some $3 D$ structure is known or predicted
Node Color

(8) colored nodes:

query proteins and first shell of interactors

(a) White nodes:

second shell of interactors
Known Interactions
- $-\mathrm{O}$ from curated databases
$\mathrm{C}-\mathrm{O}$ experimentally determined
Predicted Interactions

$\mathrm{C}-\mathrm{O}$ gene neighborhood

$\mathrm{C}-\odot$ gene fusions

-○ gene co-occurrence
Others

$\odot-\mathrm{O}$ textmining

$\odot-\odot$ co-expression

○-O protein homology

Supplementary Figure 1. String-analysis for protein-protein interaction performed on proteins whose decreased post-depletion abundance suggested primary or secondary IgY14-column depletion. Almost all proteins are known to interact with one another. Shapes and interaction type is defined by the String software and described within the figure. The primary binders were marked with red circle. 


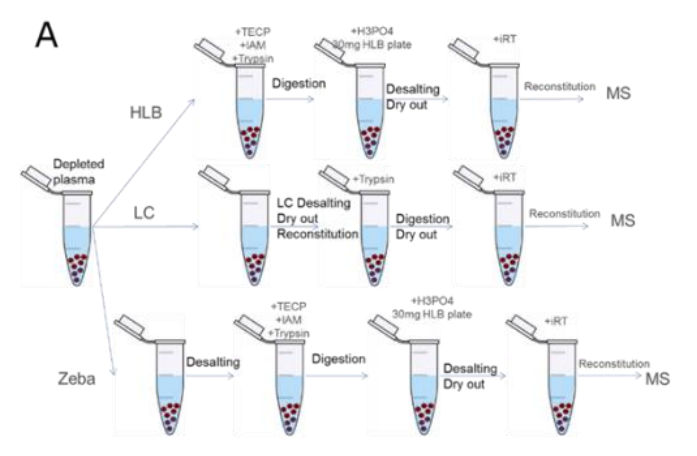

\begin{tabular}{cccc}
\hline B & HLB & Zeba & LC \\
\hline $\begin{array}{c}\text { Desalting } \\
\text { Mechanism }\end{array}$ & SPE & $\begin{array}{c}\text { Size exclusion } \\
\text { \& SPE }\end{array}$ & Reverse phase \\
Type & $\begin{array}{c}\text { Peptide } \\
\text { desalting }\end{array}$ & $\begin{array}{c}\text { Protein \& } \\
\text { peptide } \\
\text { desalting }\end{array}$ & $\begin{array}{c}\text { Protein } \\
\text { desalting }\end{array}$ \\
$\begin{array}{c}\text { Time } \\
\text { (96 samples) }\end{array}$ & 1.5 days & 3 days & 14 days \\
Automation & $* * * *$ & $* *$ & $* *$ \\
\hline
\end{tabular}

Supplementary Figure 2. Conceptual comparison of three desalting strategies. Panel A depicts the workflows for three possible desalting strategies: i) Protein digestion followed by Solid Phase Extraction using HLB (Waters) elution plates; ii) Protein desalting by size exclusion chromatography (Zeba spin columns) with subsequent digestion and Solid Phase Extraction; iii) Off-line $\mathrm{C}_{18}$-reverse phase LC-based protein desalting and dry-down ahead of tryptic digestion in an Ammonium Bicarbonate buffer. Panel B compares the approximate time required for each strategy to process 96 samples, as well as the compatibility of each strategy to automation. 
Supplementary table 1. Optimized scheduled MRM parameters for the selected proteins and peptides.

\begin{tabular}{|c|c|c|c|c|c|c|c|c|c|c|}
\hline \multirow{2}{*}{ Protein } & \multirow{2}{*}{ Peptide } & \multirow{2}{*}{$\begin{array}{c}\text { RT } \\
\text { (min) }\end{array}$} & \multicolumn{2}{|c|}{ Precursor ion } & \multirow[t]{2}{*}{ type } & \multicolumn{2}{|c|}{ Fragment ion $(\mathrm{m} / \mathrm{z})$} & \multirow[t]{2}{*}{ type } & \multirow[t]{2}{*}{ DP } & \multirow[t]{2}{*}{$\mathbf{C E}$} \\
\hline & & & light & heavy & & light & heavy & & & \\
\hline \multirow{3}{*}{ BGAL } & \multirow{3}{*}{ IDPNAWVER } & \multirow{3}{*}{14.7} & \multirow{3}{*}{550.3} & \multirow{3}{*}{555.3} & \multirow{3}{*}{+2} & 774.2 & 784.2 & y6+ & 50 & 31 \\
\hline & & & & & & 871.2 & 881.2 & $\mathrm{y} 7+$ & 50 & 25 \\
\hline & & & & & & 436.1 & 441.1 & $\mathrm{y} 72_{2+}$ & 50 & 22 \\
\hline
\end{tabular}

*RT: Retention time; CE: Collision energy; DP: Declustering potential.

*The transitions used for quantification are listed in bold. The stable isotope labeled (heavy) amino acid residues are underlined. 
Supplementary table 2. The protein name and Swiss-Prot ID of the top 14 plasma proteins depleted by the IgY14 column. Some of those high abundant proteins have several Swiss-Prot ID corresponding to different chain or regions of the same protein. The protein names of the parts belonging to the same protein were written in italics. 30

\begin{tabular}{|c|c|c|}
\hline Protein name & Swiss-Prot accession number & Gene name \\
\hline Albumin & $\mathrm{P} 02768$ & ALB \\
\hline \multicolumn{3}{|l|}{ Fibrinogen } \\
\hline alpha chain & $\underline{\mathrm{P} 02671}$ & FGA \\
\hline beta chain & $\overline{\mathrm{P} 02675}$ & FGB \\
\hline gamma chain & $\underline{\mathrm{P} 02679}$ & FGG \\
\hline Haptoglobin & $\overline{\mathrm{P} 00738}$ & HP \\
\hline Transferrin & P02787 & TF \\
\hline Apolipoprotein A1 & $\overline{\mathrm{P} 02647}$ & APOA1 \\
\hline Apolipoprotein A2 & $\overline{\mathrm{P} 02652}$ & APOA2 \\
\hline Apolipoprotein B-100 & $\overline{\mathrm{P} 04114}$ & APOB \\
\hline$\alpha-1-A n t i t r y p s i n$ & $\overline{\mathrm{P} 01009}$ & SERPINA1 \\
\hline a-2-Macroglobulin & $\overline{\mathrm{P} 01023}$ & $\mathrm{~A} 2 \mathrm{M}$ \\
\hline$\alpha-1-A c i d$ glycoprotein & $\overline{\mathrm{P} 02763}$ & ORM1 \\
\hline Complement C3 & $\mathrm{P} 01024$ & C3 \\
\hline Ig mu chain $\mathrm{C}$ region & $\mathrm{P} 01871$ & $\lg \mathrm{HM}$ \\
\hline \multicolumn{3}{|l|}{ Ig gamma chain } \\
\hline lg gamma- 1 chain $C$ region & $\underline{\mathrm{P}} 01857$ & $\operatorname{lgHG} 1$ \\
\hline Ig gamma-2 chain $C$ region & P01859 & $\operatorname{lgHG} 2$ \\
\hline Ig gamma-3 chain $C$ region & $\mathrm{P} 01860$ & $\operatorname{lgHG} 3$ \\
\hline Ig delta chain & P01880 & $\operatorname{lgHD}$ \\
\hline Ig epsilon chain $\mathrm{C}$ region & $\overline{\mathrm{P} 01854}$ & $\lg \mathrm{HE}$ \\
\hline \multicolumn{3}{|l|}{ Ig alpha chain } \\
\hline Ig alpha-1 chain & $\underline{\mathrm{P} 01876}$ & $\lg \mathrm{HA} 1$ \\
\hline Ig alpha-2 chain & $\overline{\mathrm{P} 01877}$ & $\mathrm{IgHA} 2$ \\
\hline \multicolumn{3}{|c|}{ Ig free light chains kappa and lambda } \\
\hline Ig kappa chain $\mathrm{V}-\mathrm{III}$ region $\mathrm{VH}$ & P04434 & - \\
\hline Ig kappa chain V-I region Wes & $\overline{\mathrm{P} 01611}$ & - \\
\hline Ig kappa chain $V-I$ region $C A R$ & P01596 & - \\
\hline Ig kappa chain V-III region SIE & $\overline{\mathrm{P} 01620}$ & - \\
\hline Ig kappa chain $V-I V$ region Len & $\overline{\mathrm{P} 01625}$ & - \\
\hline Ig kappa chain $V-I$ region $A G$ & $\overline{\mathrm{P} 01593}$ & - \\
\hline Ig kappa chain $V-I$ region EU & $\overline{\mathrm{P} 01598}$ & - \\
\hline Ig kappa chain $V$-III region WOL & $\overline{\mathrm{P} 01623}$ & - \\
\hline Ig kappa chain $C$ region & $\mathrm{P01834}$ & $\lg K C$ \\
\hline Ig lambda-2 chain $C$ regions & POCG05 & $\operatorname{lgLC2}$ \\
\hline Ig lambda chain V-III region LOI & $\overline{\mathrm{P} 80748}$ & - \\
\hline Ig lambda chain $\mathrm{V}$-III region $\mathrm{SH}$ & $\overline{\mathrm{P} 01714}$ & - \\
\hline Ig lambda chain $V-I V$ region Hil & $\mathrm{P} 01717$ & - \\
\hline
\end{tabular}


Supplementary table 3. Protein subsets identified with increasing amounts of depleted-and undepleted- plasma fractions injection on column. Grey cells indicate that a given protein was identified under a given condition; Clear cells indicates that a given protein was not identified.

\begin{tabular}{|c|c|c|c|c|c|c|c|c|c|c|c|c|c|}
\hline \multirow[b]{2}{*}{ Uniprot ID } & \multirow[b]{2}{*}{ Protein name } & \multicolumn{6}{|c|}{ Depleted plasma (ng on column) } & \multicolumn{6}{|c|}{ Undepleted plasma (ng on column) } \\
\hline & & 63 & 125 & 250 & 500 & 1000 & 2000 & 63 & 125 & 250 & 500 & 1000 & 2000 \\
\hline B9A064 & IGLL5_HUMAN & & & & & & & & & & & & \\
\hline 000187 & MASP2_HUMAN & & & & & & & & & & & & \\
\hline 000299 & CLIC1_HUMAN & & & & & & & & & & & & \\
\hline 000391 & QSOX1_HUMAN & & & & & & & & & & & & \\
\hline 000410 & IPO5_HUMAN & & & & & & & & & & & & \\
\hline 000429 & DNM1L_HUMAN & & & & & & & & & & & & \\
\hline 000533 & CHL1_HUMAN & & & & & & & & & & & & \\
\hline 014618 & CCS_HUMAN & & & & & & & & & & & & \\
\hline 014776 & TCRG1_HUMAN & & & & & & & & & & & & \\
\hline 014791 & APOL1_HUMAN & & & & & & & & & & & & \\
\hline 014818 & PSA7_HUMAN & & & & & & & & & & & & \\
\hline 014980 & XPO1_HUMAN & & & & & & & & & & & & \\
\hline 015067 & PUR4_HUMAN & & & & & & & & & & & & \\
\hline 015230 & LAMA5_HUMAN & & & & & & & & & & & & \\
\hline 015269 & SPTC1_HUMAN & & & & & & & & & & & & \\
\hline 015347 & HMGB3_HUMAN & & & & & & & & & & & & \\
\hline 015400 & STX7_HUMAN & & & & & & & & & & & & \\
\hline 015523 & DDX3Y_HUMAN & & & & & & & & & & & & \\
\hline 043175 & SERA_HUMAN & & & & & & & & & & & & \\
\hline 043396 & TXNL1_HUMAN & & & & & & & & & & & & \\
\hline 043505 & B3GN1_HUMAN & & & & & & & & & & & & \\
\hline 043583 & DENR_HUMAN & & & & & & & & & & & & \\
\hline 043598 & DNPH1_HUMAN & & & & & & & & & & & & \\
\hline 043776 & SYNC_HUMAN & & & & & & & & & & & & \\
\hline 043866 & CD5L_HUMAN & & & & & & & & & & & & \\
\hline 060306 & AQR_HUMAN & & & & & & & & & & & & \\
\hline 060610 & DIAP1_HUMAN & & & & & & & & & & & & \\
\hline 060664 & PLIN3_HUMAN & & & & & & & & & & & & \\
\hline 060716 & CTND1_HUMAN & & & & & & & & & & & & \\
\hline 075083 & WDR1_HUMAN & & & & & & & & & & & & \\
\hline 075131 & CPNE3_HUMAN & & & & & & & & & & & & \\
\hline 075144 & ICOSL_HUMAN & & & & & & & & & & & & \\
\hline 075369 & FLNB_HUMAN & & & & & & & & & & & & \\
\hline 075376 & NCOR1_HUMAN & & & & & & & & & & & & \\
\hline 075380 & NDUS6_HUMAN & & & & & & & & & & & & \\
\hline 075390 & CISY_HUMAN & & & & & & & & & & & & \\
\hline
\end{tabular}




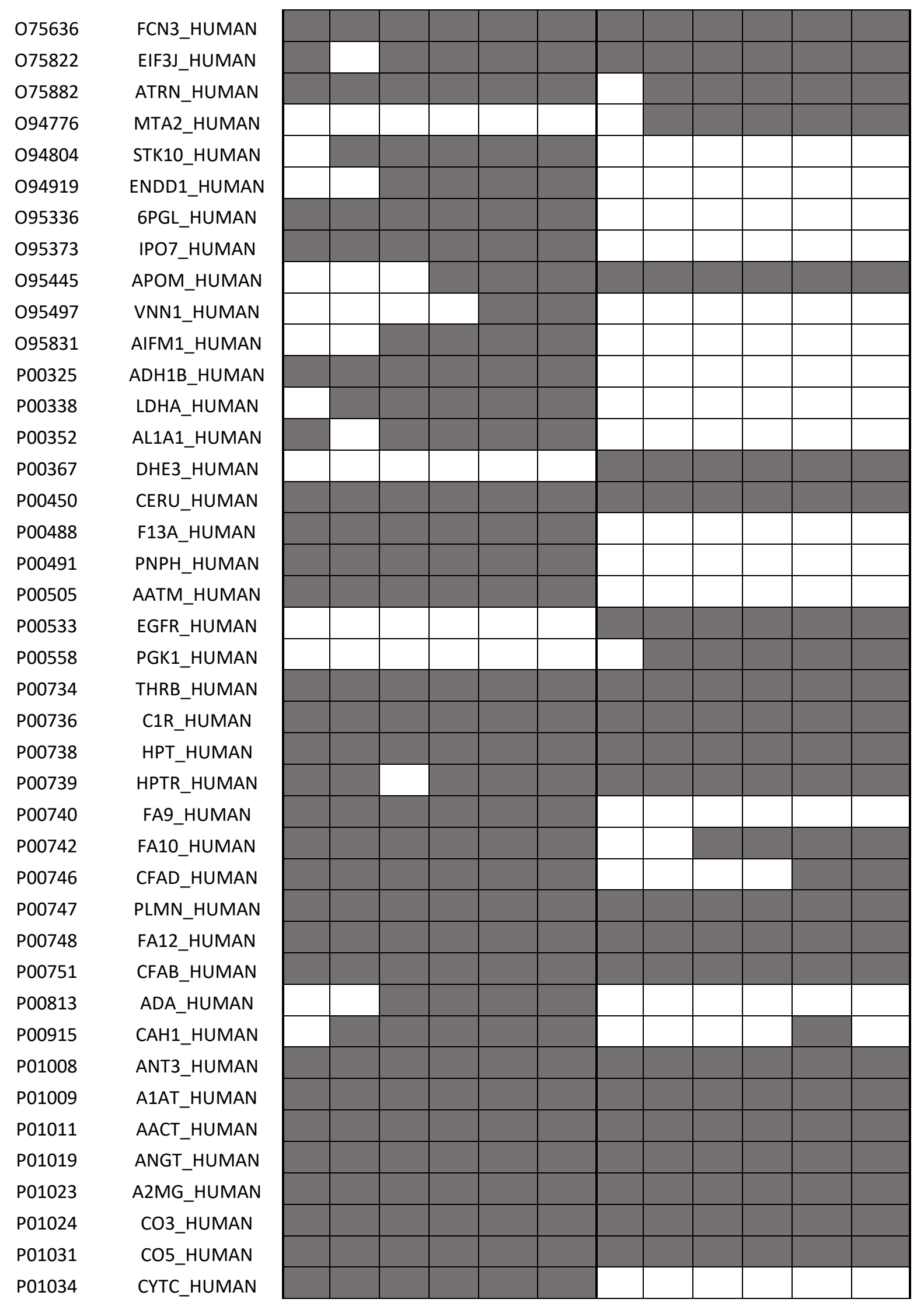




$\begin{array}{lc}\text { P01042 } & \text { KNG1_HUMAN } \\ \text { P01344 } & \text { IGF2_HUMAN } \\ \text { P01591 } & \text { IGJHUMAN } \\ \text { P01593 } & \text { KV101_HUMAN } \\ \text { P01594 } & \text { KV102_HUMAN } \\ \text { P01597 } & \text { KV105_HUMAN } \\ \text { P01608 } & \text { KV116_HUMAN } \\ \text { P01621 } & \text { KV303_HUMAN } \\ \text { P01714 } & \text { LV301_HUMAN } \\ \text { P01717 } & \text { LV403_HUMAN } \\ \text { P01764 } & \text { HV303_HUMAN } \\ \text { P01780 } & \text { HV319_HUMAN } \\ \text { P01781 } & \text { HV320_HUMAN } \\ \text { P01834 } & \text { IGKC_HUMAN } \\ \text { P01857 } & \text { IGHG1_HUMAN } \\ \text { P01859 } & \text { IGHG2_HUMAN } \\ \text { P01860 } & \text { IGHG3_HUMAN } \\ \text { P01861 } & \text { IGHG4_HUMAN } \\ \text { P01871 } & \text { IGHM_HUMAN } \\ \text { P01876 } & \text { IGHA1_HUMAN } \\ \text { P01877 } & \text { IGHA2_HUMAN } \\ \text { P01880 } & \text { IGHD_HUMAN } \\ \text { P02042 } & \text { HBD_HUMAN } \\ \text { P02461 } & \text { CO3A1_HUMAN } \\ \text { P02545 } & \text { LMNA_HUMAN } \\ \text { P02647 } & \text { APOA1_HUMAN } \\ \text { P02649 } & \text { APOE_HUMAN } \\ \text { P02652 } & \text { APOA2_HUMAN } \\ \text { P02654 } & \text { APOC1_HUMAN } \\ \text { P02655 } & \text { APOC2_HUMAN } \\ \text { P02656 } & \text { APOC3_HUMAN } \\ \text { P02671 } & \text { FIBA_HUMAN } \\ \text { P02675 } & \text { FIBB_HUMAN } \\ \text { P02679 } & \text { FIBG_HUMAN } \\ \text { P02730 } & \text { B3AT_HUMAN } \\ \text { P02741 } 02746 & \text { CRP_HUMAN } \\ \text { P02743 } 02747 & \text { SAMP_HUMAN } \\ \text { P02748 } & \text { C1QA_HUMAN_HUMAN } \\ & \text { CO9HUMAN }\end{array}$

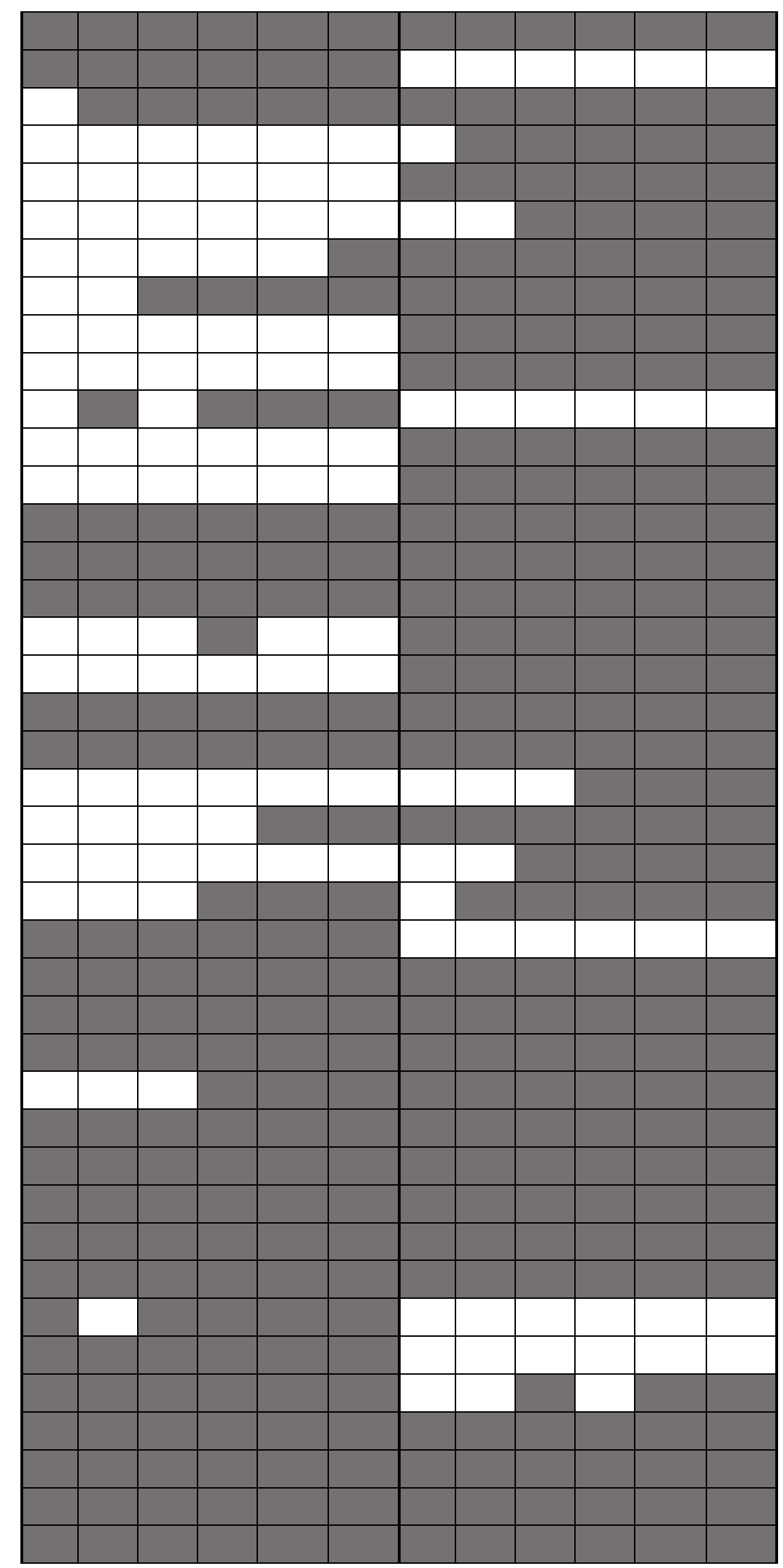




\begin{tabular}{|c|c|}
\hline P02749 & APOH_HUMAN \\
\hline P02750 & A2GL_HUMAN \\
\hline P02751 & FINC_HUMAN \\
\hline P02753 & RET4_HUMAN \\
\hline P02760 & AMBP_HUMAN \\
\hline P02763 & A1AG1_HUMAN \\
\hline P02765 & FETUA_HUMAN \\
\hline P02766 & TTHY_HUMAN \\
\hline P02768 & ALBU_HUMAN \\
\hline P02774 & VTDB_HUMAN \\
\hline P02775 & CXCL7_HUMAN \\
\hline P02787 & TRFE_HUMAN \\
\hline P02790 & HEMO_HUMAN \\
\hline P02794 & FRIH_HUMAN \\
\hline P03951 & FA11_HUMAN \\
\hline P03952 & KLKB1_HUMAN \\
\hline P04003 & C4BPA_HUMAN \\
\hline P04004 & VTNC_HUMAN \\
\hline P04040 & CATA_HUMAN \\
\hline P04054 & PA21B_HUMAN \\
\hline P04070 & PROC_HUMAN \\
\hline P04075 & ALDOA_HUMAN \\
\hline P04083 & ANXA1_HUMAN \\
\hline P04114 & APOB_HUMAN \\
\hline P04179 & SODM_HUMAN \\
\hline P04180 & LCAT_HUMAN \\
\hline P04196 & HRG_HUMAN \\
\hline P04211 & LV001_HUMAN \\
\hline P04217 & A1BG_HUMAN \\
\hline P04220 & MUCB_HUMAN \\
\hline P04264 & K2C1_HUMAN \\
\hline P04275 & VWF_HUMAN \\
\hline P04278 & SHBG_HUMAN \\
\hline P04406 & G3P_HUMAN \\
\hline P04430 & KV122_HUMAN \\
\hline P04433 & KV309_HUMAN \\
\hline P05019 & IGF1_HUMAN \\
\hline P05062 & ALDOB_HUMAN \\
\hline P05067 & A4_HUMAN \\
\hline P05090 & APOD_HUMAN \\
\hline P05091 & ALDH2_HUMAN \\
\hline
\end{tabular}

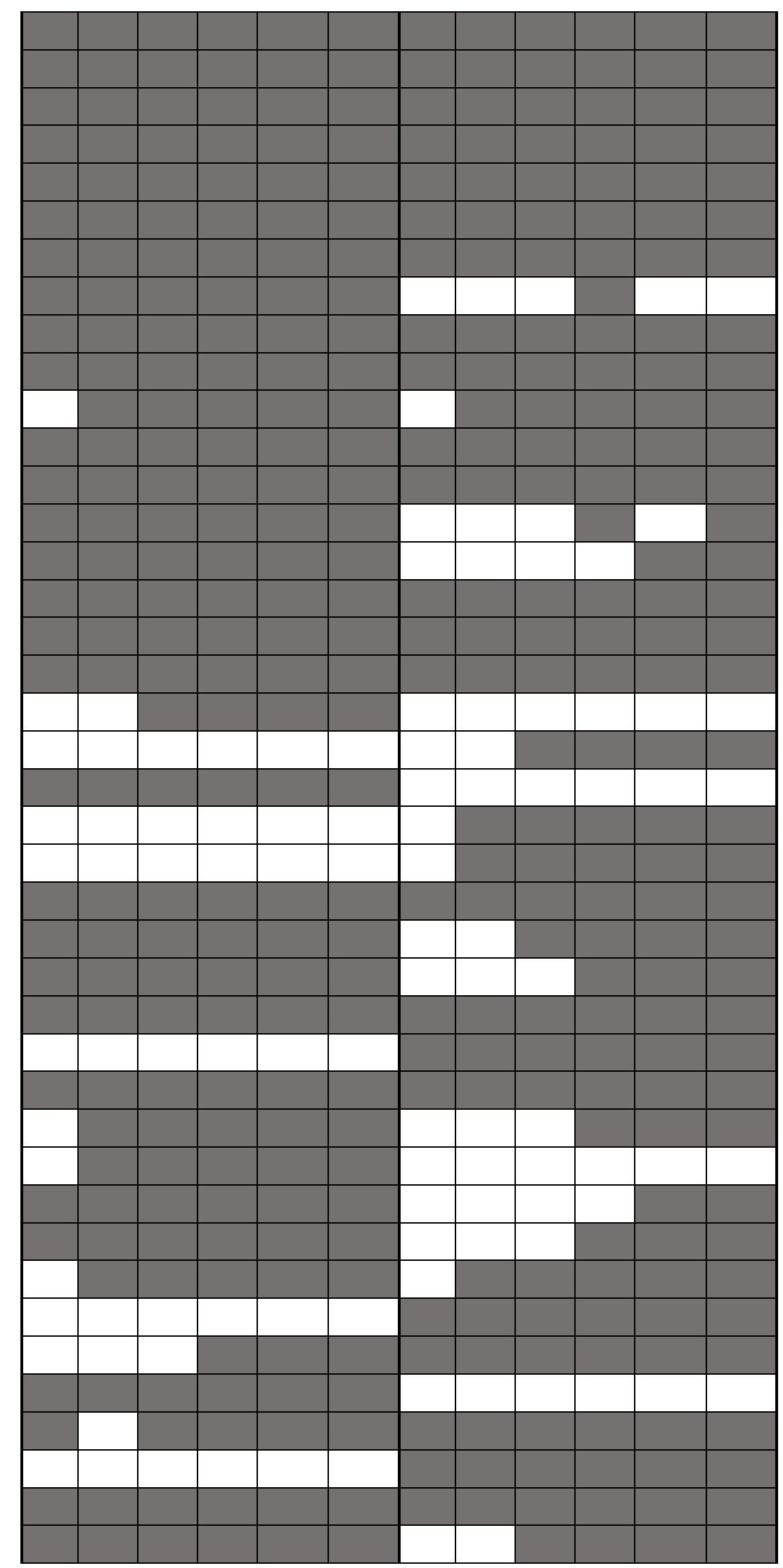




\begin{tabular}{|c|c|}
\hline P05109 & S10A8_HUMAN \\
\hline P05121 & PAI1_HUMAN \\
\hline P05155 & IC1_HUMAN \\
\hline P05156 & CFAI_HUMAN \\
\hline P05160 & F13B_HUMAN \\
\hline P05164 & PERM_HUMAN \\
\hline P05165 & PCCA_HUMAN \\
\hline P05166 & PCCB_HUMAN \\
\hline P05186 & PPBT_HUMAN \\
\hline P05362 & ICAM1_HUMAN \\
\hline P05413 & FABPH_HUMAN \\
\hline P05452 & TETN_HUMAN \\
\hline P05543 & THBG_HUMAN \\
\hline P05546 & HEP2_HUMAN \\
\hline P05556 & ITB1_HUMAN \\
\hline P05787 & K2C8_HUMAN \\
\hline P06132 & DCUP_HUMAN \\
\hline P06276 & CHLE_HUMAN \\
\hline P06310 & KV206_HUMAN \\
\hline P06331 & HV209_HUMAN \\
\hline P06396 & GELS_HUMAN \\
\hline P06576 & ATPB_HUMAN \\
\hline P06681 & CO2_HUMAN \\
\hline P06702 & S10A9_HUMAN \\
\hline P06703 & S10A6_HUMAN \\
\hline P06727 & APOA4_HUMAN \\
\hline P06733 & ENOA_HUMAN \\
\hline P06737 & PYGL_HUMAN \\
\hline P06744 & G6PI_HUMAN \\
\hline P06748 & NPM_HUMAN \\
\hline P06865 & HEXA_HUMAN \\
\hline P07195 & LDHB_HUMAN \\
\hline P07225 & PROS_HUMAN \\
\hline P07237 & PDIA1_HUMAN \\
\hline P07333 & CSF1R_HUMAN \\
\hline P07339 & CATD_HUMAN \\
\hline P07355 & ANXA2_HUMAN \\
\hline P07357 & CO8A_HUMAN \\
\hline P07358 & CO8B_HUMAN \\
\hline P07359 & GP1BA_HUMAN \\
\hline P07360 & CO8G_HUMAN \\
\hline
\end{tabular}

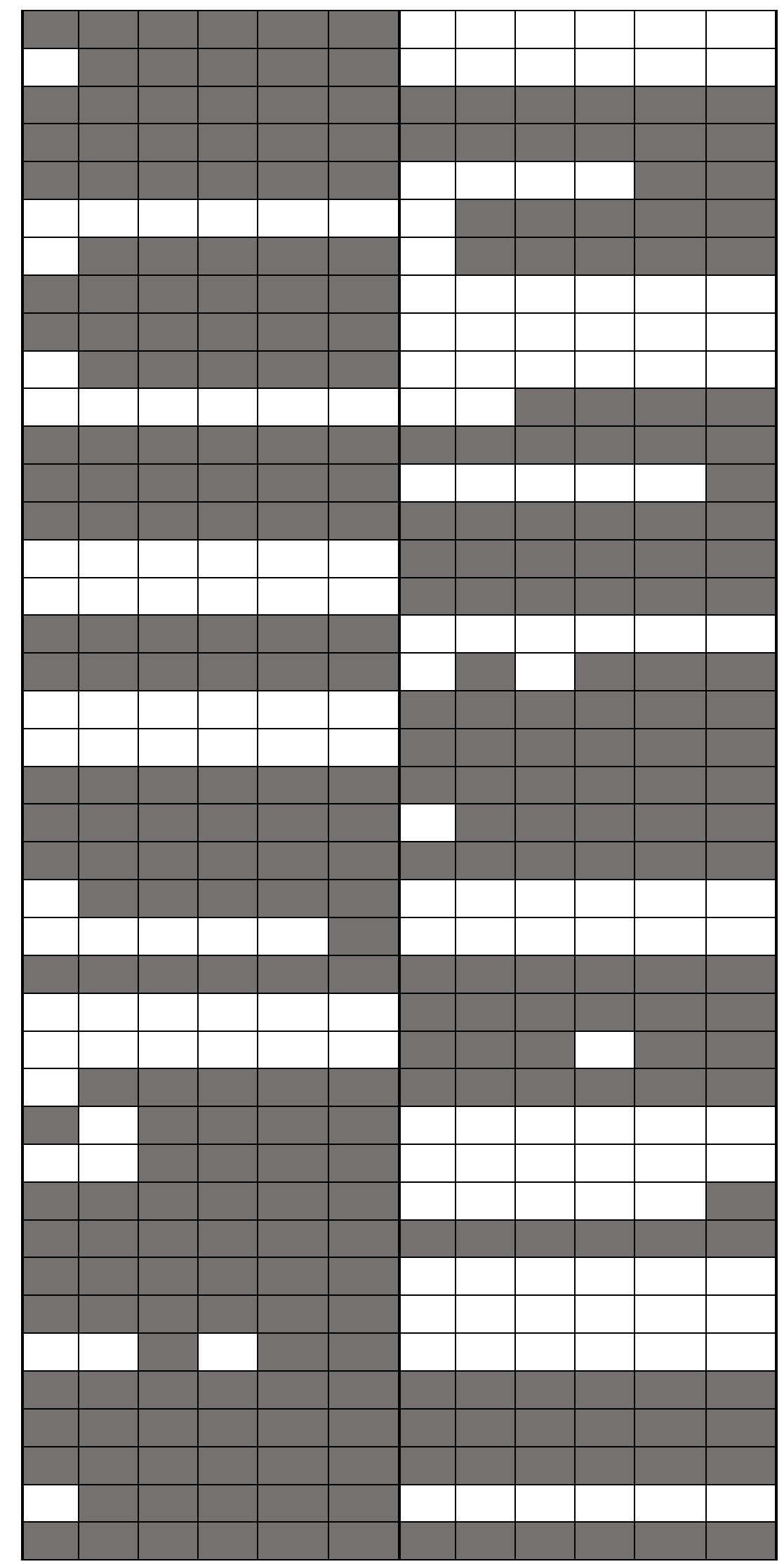




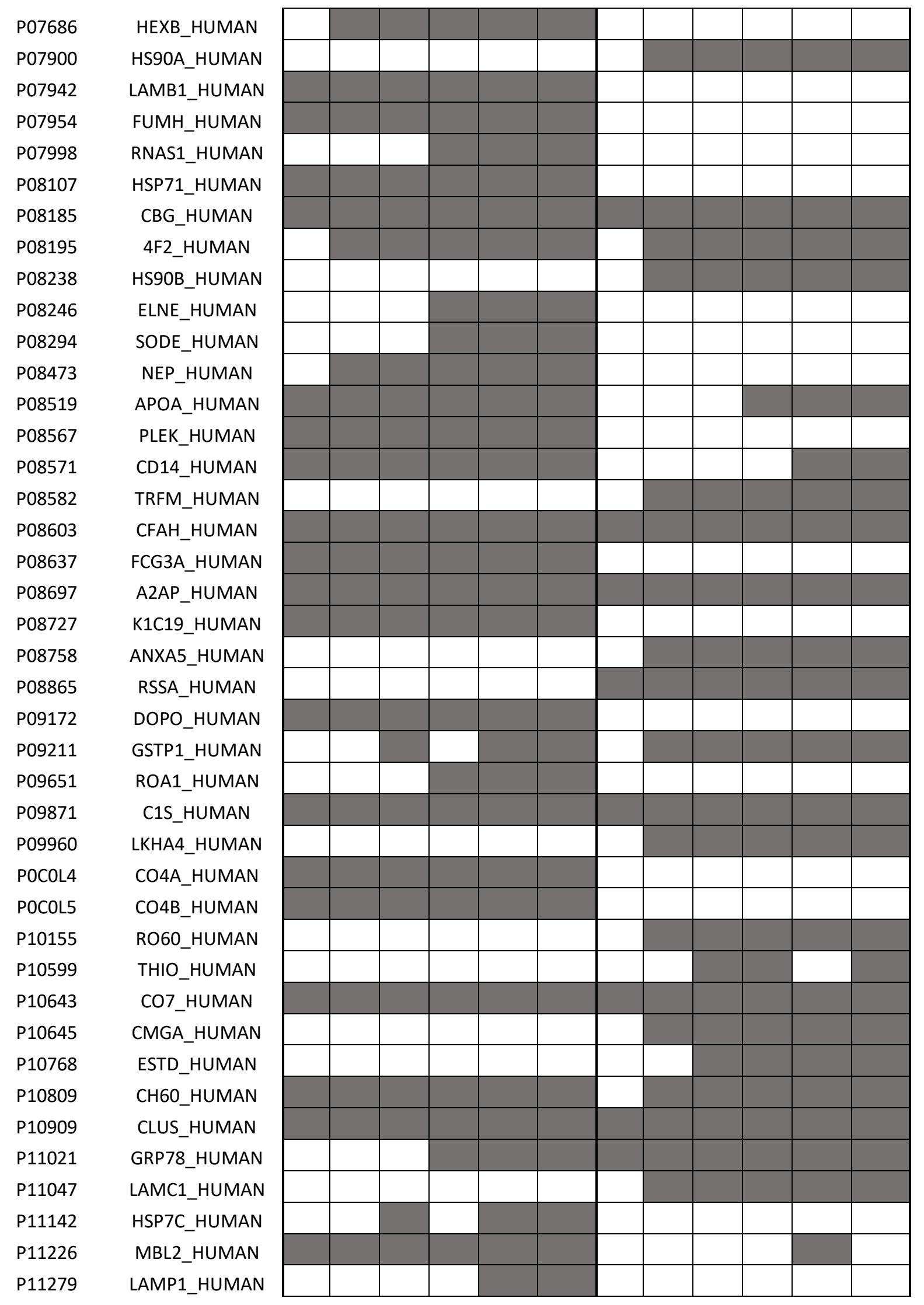




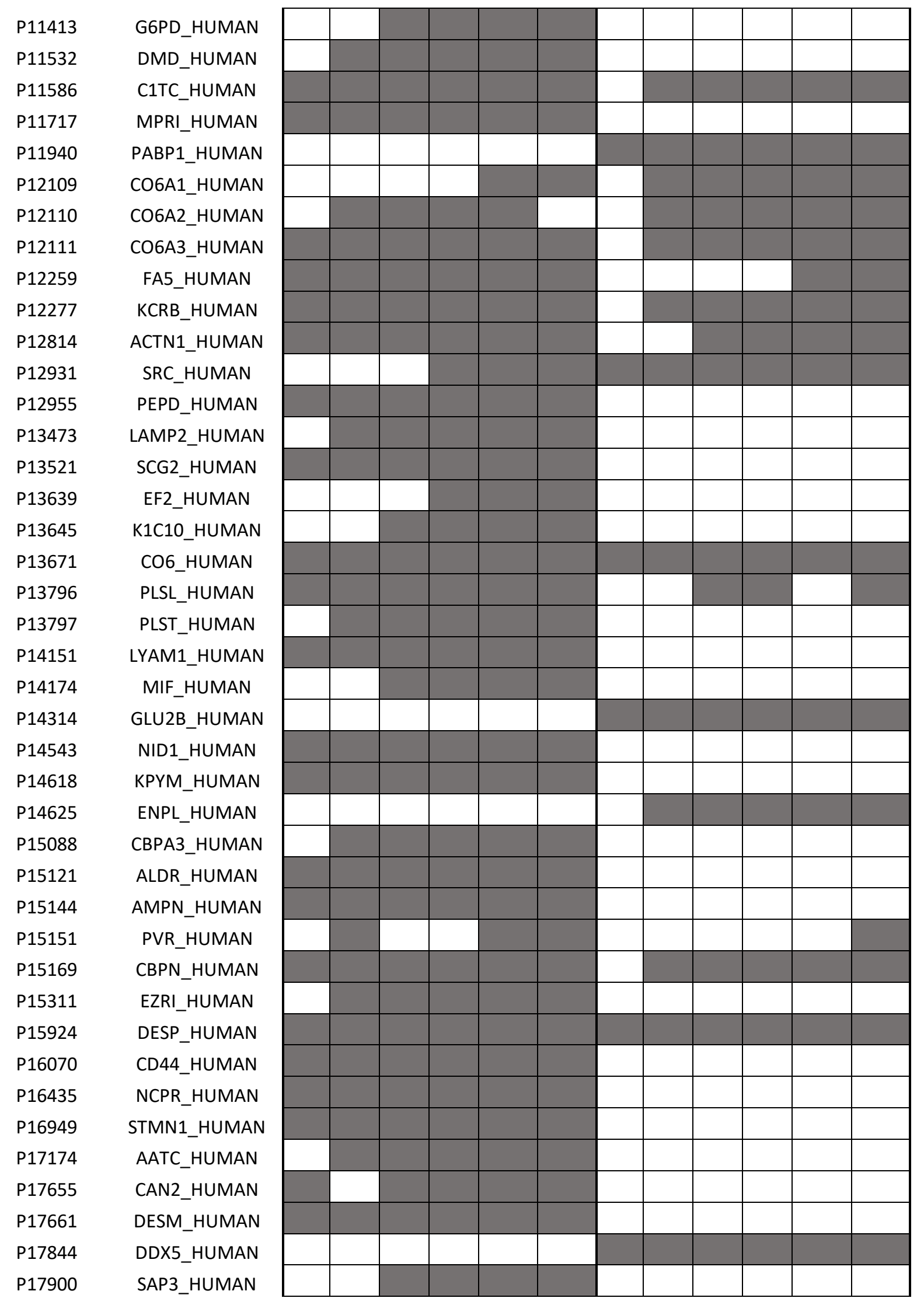




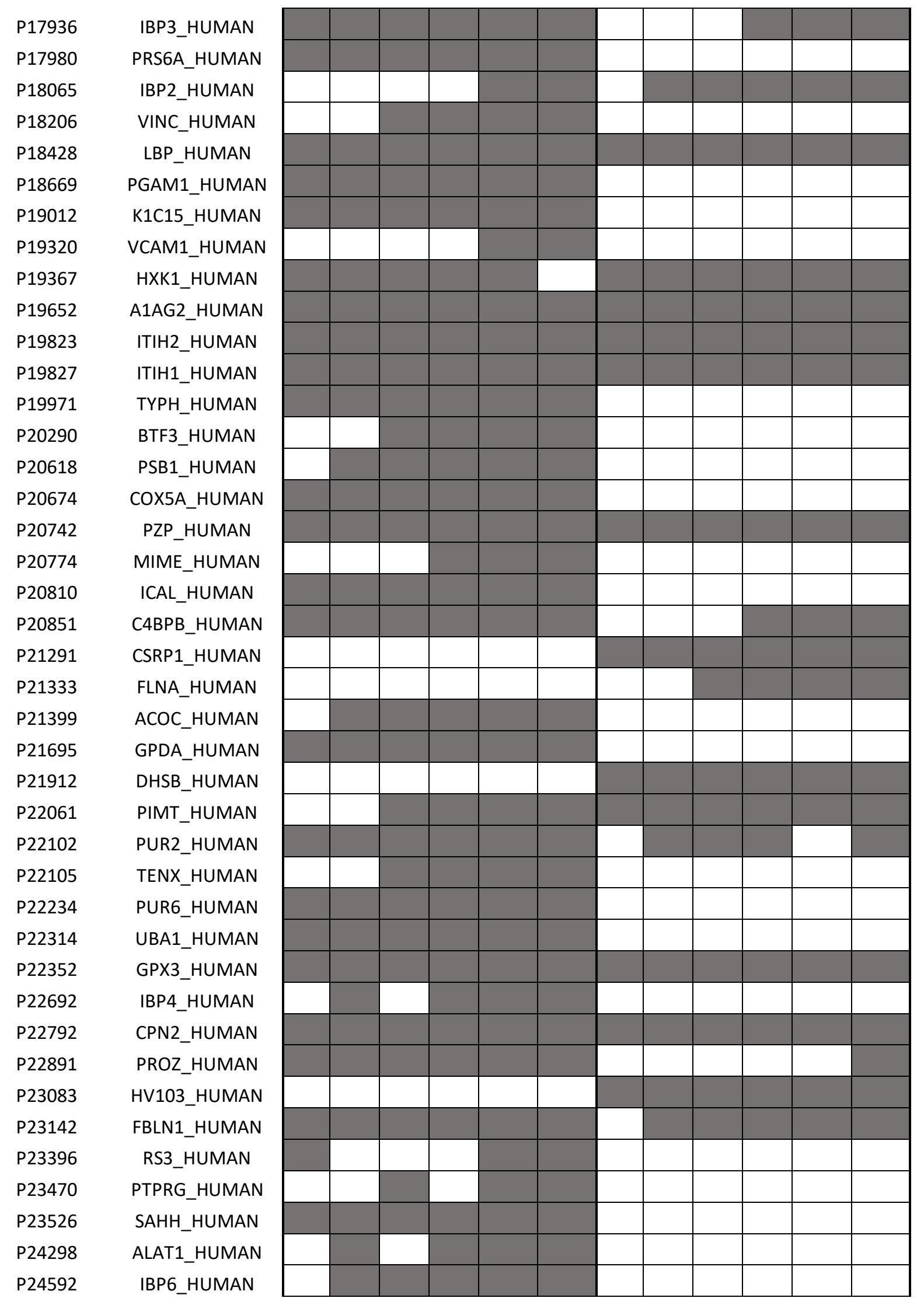




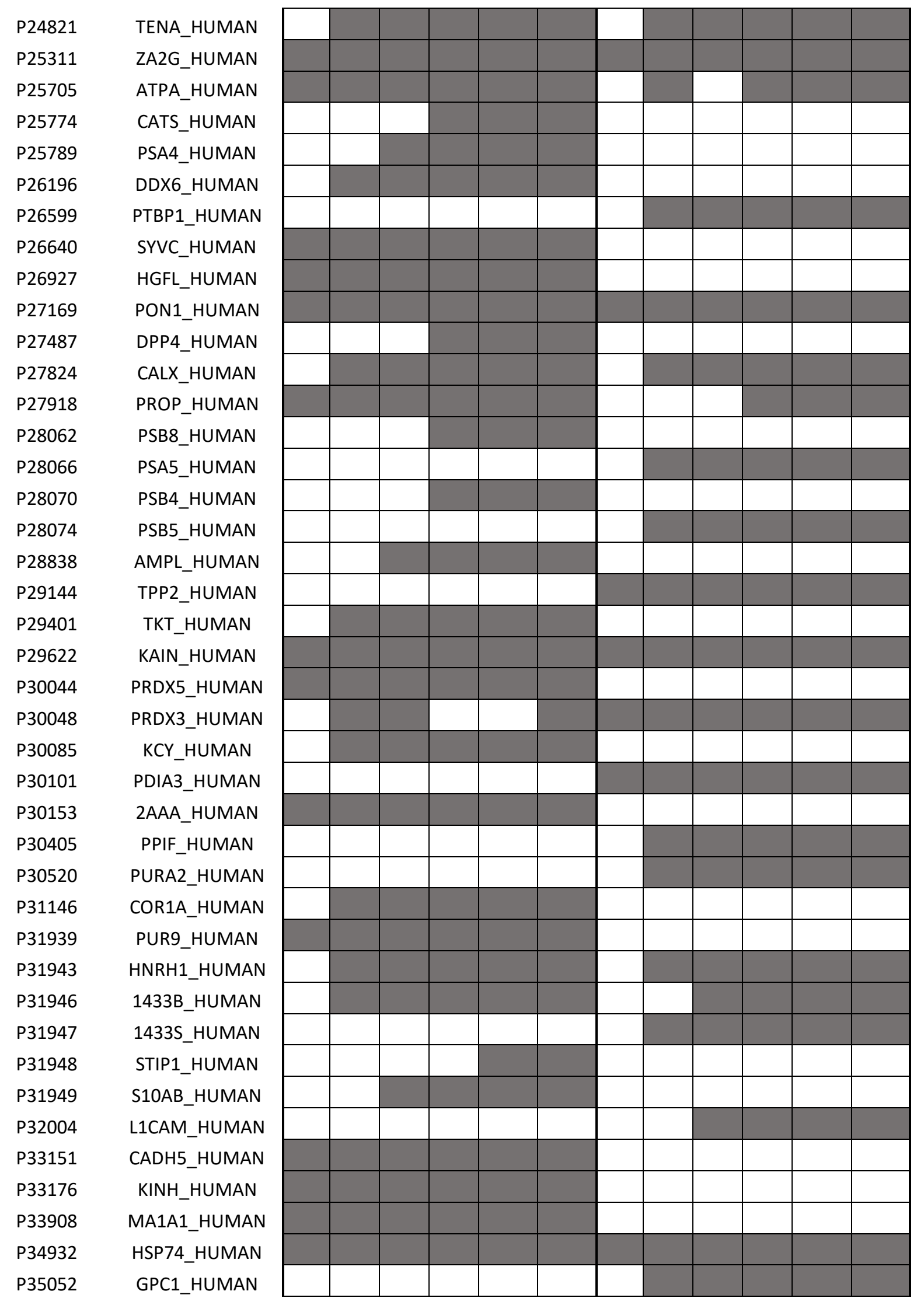




\begin{tabular}{|c|c|}
\hline P35232 & PHB_HUMAN \\
\hline P35237 & SPB6_HUMAN \\
\hline P35442 & TSP2_HUMAN \\
\hline P35520 & CBS_HUMAN \\
\hline P35542 & SAA4_HUMAN \\
\hline P35573 & GDE_HUMAN \\
\hline P35579 & MYH9_HUMAN \\
\hline P35754 & GLRX1_HUMAN \\
\hline P35858 & ALS_HUMAN \\
\hline P35900 & K1C20_HUMAN \\
\hline P35908 & K22E_HUMAN \\
\hline P36222 & CH3L1_HUMAN \\
\hline P36542 & ATPG_HUMAN \\
\hline P36871 & PGM1_HUMAN \\
\hline P36873 & PP1G_HUMAN \\
\hline P36955 & PEDF_HUMAN \\
\hline P36980 & FHR2_HUMAN \\
\hline P37802 & TAGL2_HUMAN \\
\hline P38606 & VATA_HUMAN \\
\hline P38646 & GRP75_HUMAN \\
\hline P39023 & RL3_HUMAN \\
\hline P39059 & COFA1_HUMAN \\
\hline P39060 & COIA1_HUMAN \\
\hline P40227 & TCPZ_HUMAN \\
\hline P40763 & STAT3_HUMAN \\
\hline P40925 & MDHC_HUMAN \\
\hline P41250 & SYG_HUMAN \\
\hline P42126 & ECl1_HUMAN \\
\hline P42574 & CASP3_HUMAN \\
\hline P42765 & THIM_HUMAN \\
\hline P43121 & MUC18_HUMAN \\
\hline P43243 & MATR3_HUMAN \\
\hline P43251 & BTD_HUMAN \\
\hline P43490 & NAMPT_HUMAN \\
\hline P43652 & AFAM_HUMAN \\
\hline P45974 & UBP5_HUMAN \\
\hline P46821 & MAP1B_HUMAN \\
\hline P46939 & UTRO_HUMAN \\
\hline P47756 & CAPZB_HUMAN \\
\hline P47897 & SYQ_HUMAN \\
\hline P48047 & ATPO_HUMAN \\
\hline
\end{tabular}

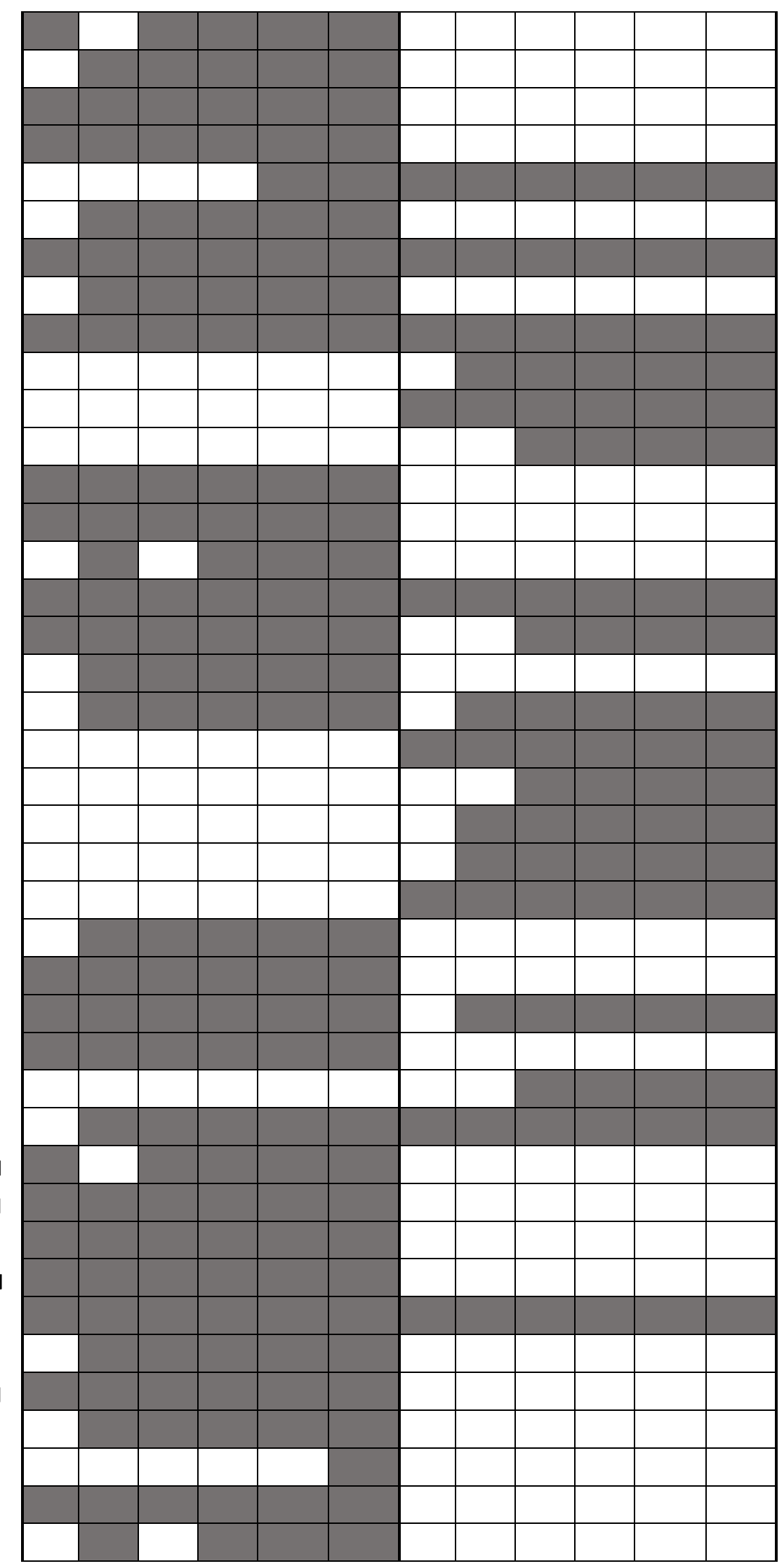




\begin{tabular}{|c|c|}
\hline P48163 & MAOX_HUMAN \\
\hline P48506 & GSH1_HUMAN \\
\hline P48681 & NEST_HUMAN \\
\hline P48740 & MASP1_HUMAN \\
\hline P49005 & DPOD2_HUMAN \\
\hline P49189 & AL9A1_HUMAN \\
\hline P49321 & NASP_HUMAN \\
\hline P49327 & FAS_HUMAN \\
\hline P49588 & SYAC_HUMAN \\
\hline P49591 & SYSC_HUMAN \\
\hline P49736 & MCM2_HUMAN \\
\hline P49753 & ACOT2_HUMAN \\
\hline P49908 & SEPP1_HUMAN \\
\hline P50281 & MMP14_HUMAN \\
\hline P50395 & GDIB_HUMAN \\
\hline P50440 & GATM_HUMAN \\
\hline P50552 & VASP_HUMAN \\
\hline P50895 & BCAM_HUMAN \\
\hline P50914 & RL14_HUMAN \\
\hline P50990 & TCPQ_HUMAN \\
\hline P50991 & TCPD_HUMAN \\
\hline P51149 & RAB7A_HUMAN \\
\hline P51570 & GALK1_HUMAN \\
\hline P51610 & HCFC1_HUMAN \\
\hline P51858 & HDGF_HUMAN \\
\hline P51884 & LUM_HUMAN \\
\hline P52209 & 6PGD_HUMAN \\
\hline P52272 & HNRPM_HUMAN \\
\hline P52565 & GDIR1_HUMAN \\
\hline P52701 & MSH6_HUMAN \\
\hline P52907 & CAZA1_HUMAN \\
\hline P52948 & NUP98_HUMAN \\
\hline P53634 & CATC_HUMAN \\
\hline P54108 & CRIS3_HUMAN \\
\hline P54136 & SYRC_HUMAN \\
\hline P54577 & SYYC_HUMAN \\
\hline P54578 & UBP14_HUMAN \\
\hline P54760 & EPHB4_HUMAN \\
\hline P54802 & ANAG_HUMAN \\
\hline P55056 & APOC4_HUMAN \\
\hline P55058 & PLTP_HUMAN \\
\hline
\end{tabular}

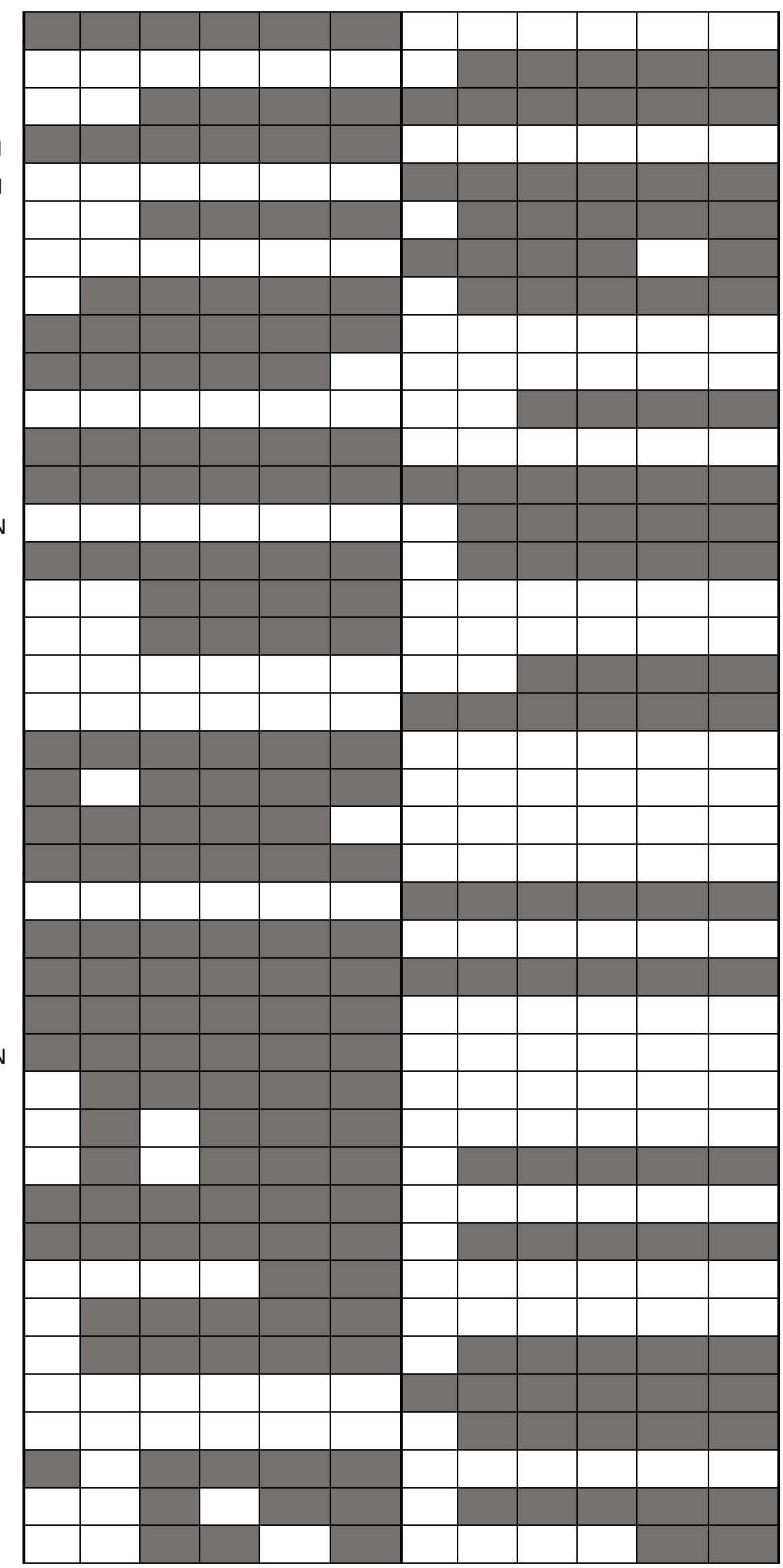




\begin{tabular}{|c|c|}
\hline P55072 & TERA_HUMAN \\
\hline P55209 & NP1L1_HUMAN \\
\hline P55285 & CADH6_HUMAN \\
\hline P55290 & CAD13_HUMAN \\
\hline P55786 & PSA_HUMAN \\
\hline P55957 & BID_HUMAN \\
\hline P58107 & EPIPL_HUMAN \\
\hline P60660 & MYL6_HUMAN \\
\hline P60842 & IF4A1_HUMAN \\
\hline P60981 & DEST_HUMAN \\
\hline P61088 & UBE2N_HUMAN \\
\hline P61158 & ARP3_HUMAN \\
\hline P61313 & RL15_HUMAN \\
\hline P61764 & STXB1_HUMAN \\
\hline P61769 & B2MG_HUMAN \\
\hline P62158 & CALM_HUMAN \\
\hline P62258 & 1433E_HUMAN \\
\hline P62263 & RS14_HUMAN \\
\hline P62314 & SMD1_HUMAN \\
\hline P62701 & RS4X_HUMAN \\
\hline P62826 & RAN_HUMAN \\
\hline P62857 & RS28_HUMAN \\
\hline P62942 & FKB1A_HUMAN \\
\hline P62993 & GRB2_HUMAN \\
\hline P63104 & 1433Z_HUMAN \\
\hline P63313 & TYB10_HUMAN \\
\hline P67809 & YBOX1_HUMAN \\
\hline P68366 & TBA4A_HUMAN \\
\hline P68871 & HBB_HUMAN \\
\hline P69905 & HBA_HUMAN \\
\hline P78347 & GTF2I_HUMAN \\
\hline P78527 & PRKDC_HUMAN \\
\hline P80108 & PHLD_HUMAN \\
\hline P84243 & H33_HUMAN \\
\hline P98160 & PGBM_HUMAN \\
\hline Q00610 & CLH1_HUMAN \\
\hline Q00839 & HNRPU_HUMAN \\
\hline Q01082 & SPTB2_HUMAN \\
\hline Q01459 & DIAC_HUMAN \\
\hline Q02790 & FKBP4_HUMAN \\
\hline Q02809 & PLOD1_HUMAN \\
\hline
\end{tabular}

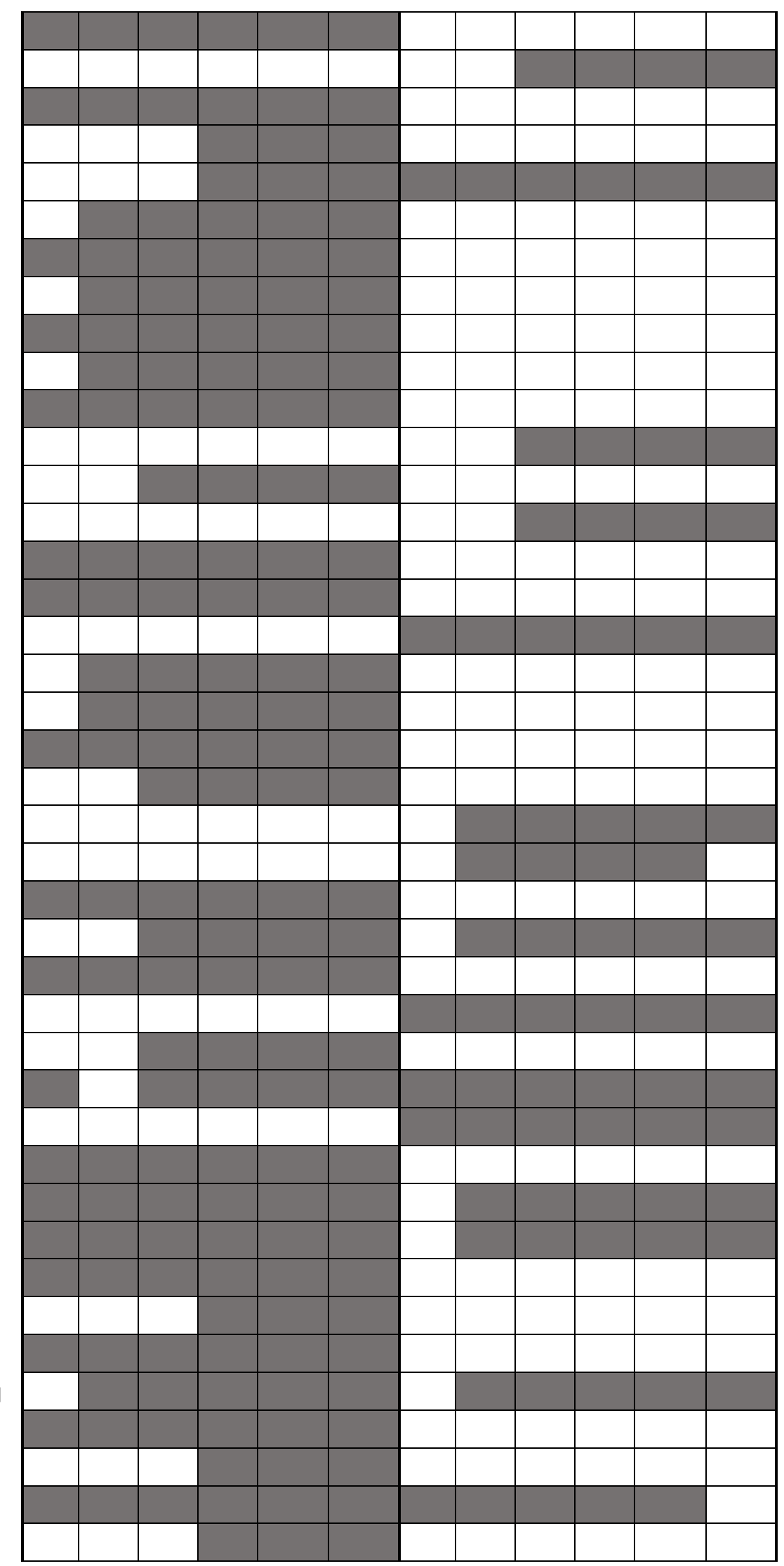




\begin{tabular}{|c|c|}
\hline Q02880 & TOP2B_HUMAN \\
\hline Q02952 & AKA12_HUMAN \\
\hline Q03154 & ACY1_HUMAN \\
\hline Q03591 & FHR1_HUMAN \\
\hline Q04756 & HGFA_HUMAN \\
\hline Q04917 & 1433F_HUMAN \\
\hline Q05397 & FAK1_HUMAN \\
\hline Q05707 & COEA1_HUMAN \\
\hline Q06033 & ITIH3_HUMAN \\
\hline Q07960 & RHG01_HUMAN \\
\hline Q08209 & PP2BA_HUMAN \\
\hline Q08257 & QOR_HUMAN \\
\hline Q08380 & LG3BP_HUMAN \\
\hline Q09666 & AHNK_HUMAN \\
\hline Q10567 & AP1B1_HUMAN \\
\hline Q10588 & BST1_HUMAN \\
\hline Q12805 & FBLN3_HUMAN \\
\hline Q12841 & FSTL1_HUMAN \\
\hline Q12906 & ILF3_HUMAN \\
\hline Q12931 & TRAP1_HUMAN \\
\hline Q13045 & FLII_HUMAN \\
\hline Q13103 & SPP24_HUMAN \\
\hline Q13185 & CBX3_HUMAN \\
\hline Q13200 & PSMD2_HUMAN \\
\hline Q13232 & NDK3_HUMAN \\
\hline Q13442 & HAP28_HUMAN \\
\hline Q13451 & FKBP5_HUMAN \\
\hline Q13557 & KCC2D_HUMAN \\
\hline Q13564 & ULA1_HUMAN \\
\hline Q13576 & IQGA2_HUMAN \\
\hline Q13740 & CD166_HUMAN \\
\hline Q13765 & NACA_HUMAN \\
\hline Q13790 & APOF_HUMAN \\
\hline Q14012 & KCC1A_HUMAN \\
\hline Q14103 & HNRPD_HUMAN \\
\hline Q14126 & DSG2_HUMAN \\
\hline Q14203 & DCTN1_HUMAN \\
\hline Q14204 & DYHC1_HUMAN \\
\hline Q14247 & SRC8_HUMAN \\
\hline Q14258 & TRI25_HUMAN \\
\hline Q14289 & FAK2_HUMAN \\
\hline
\end{tabular}

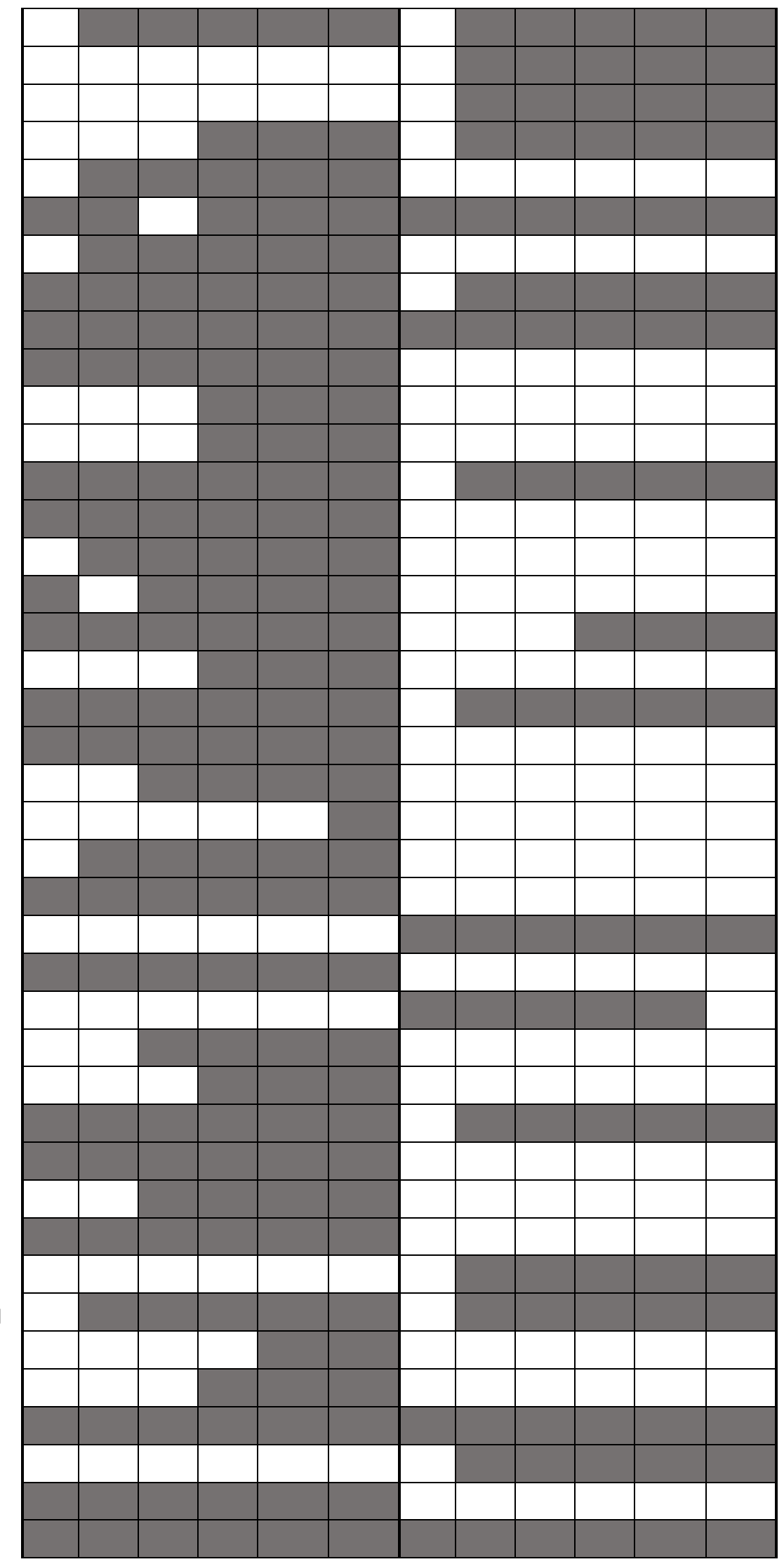




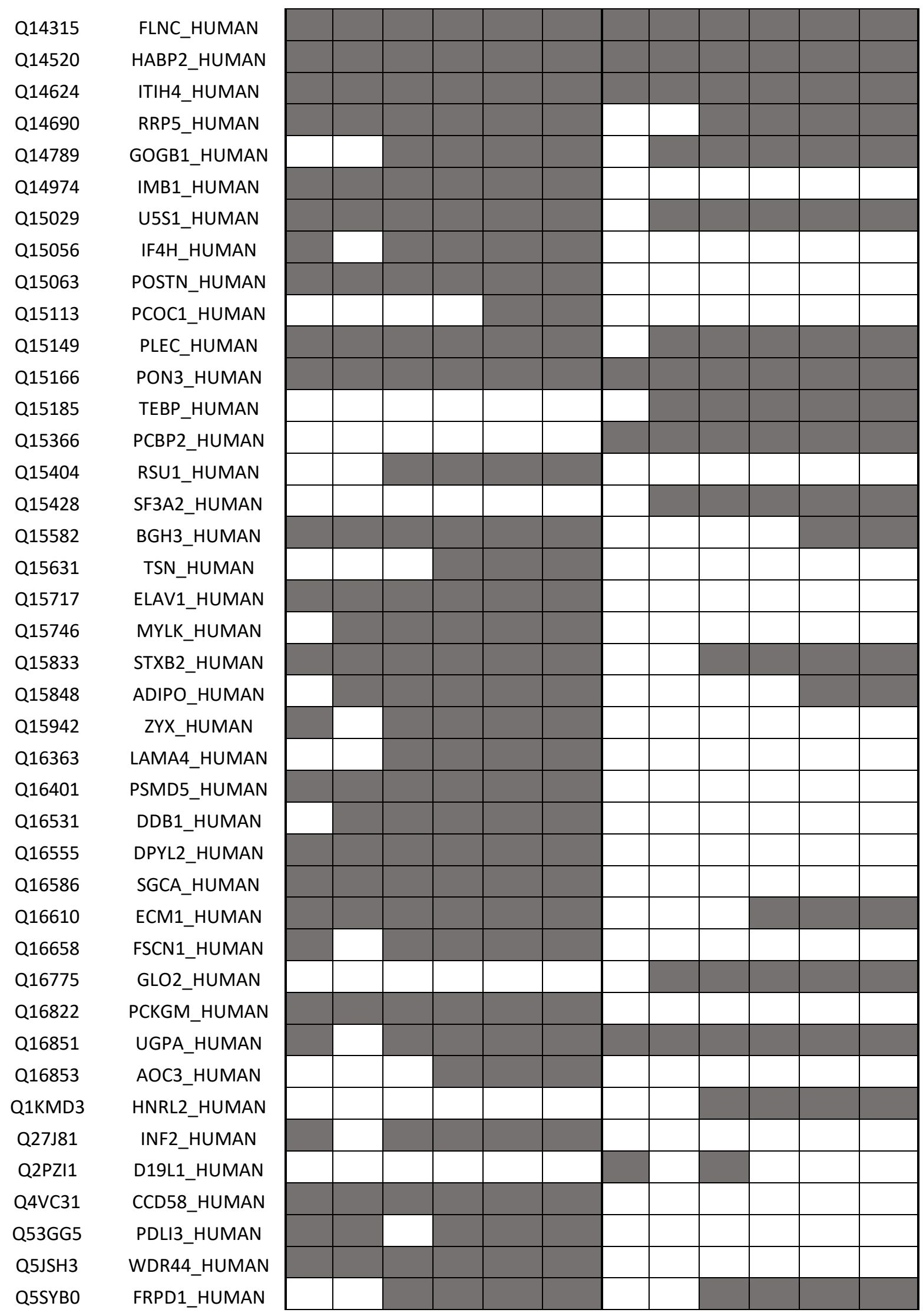




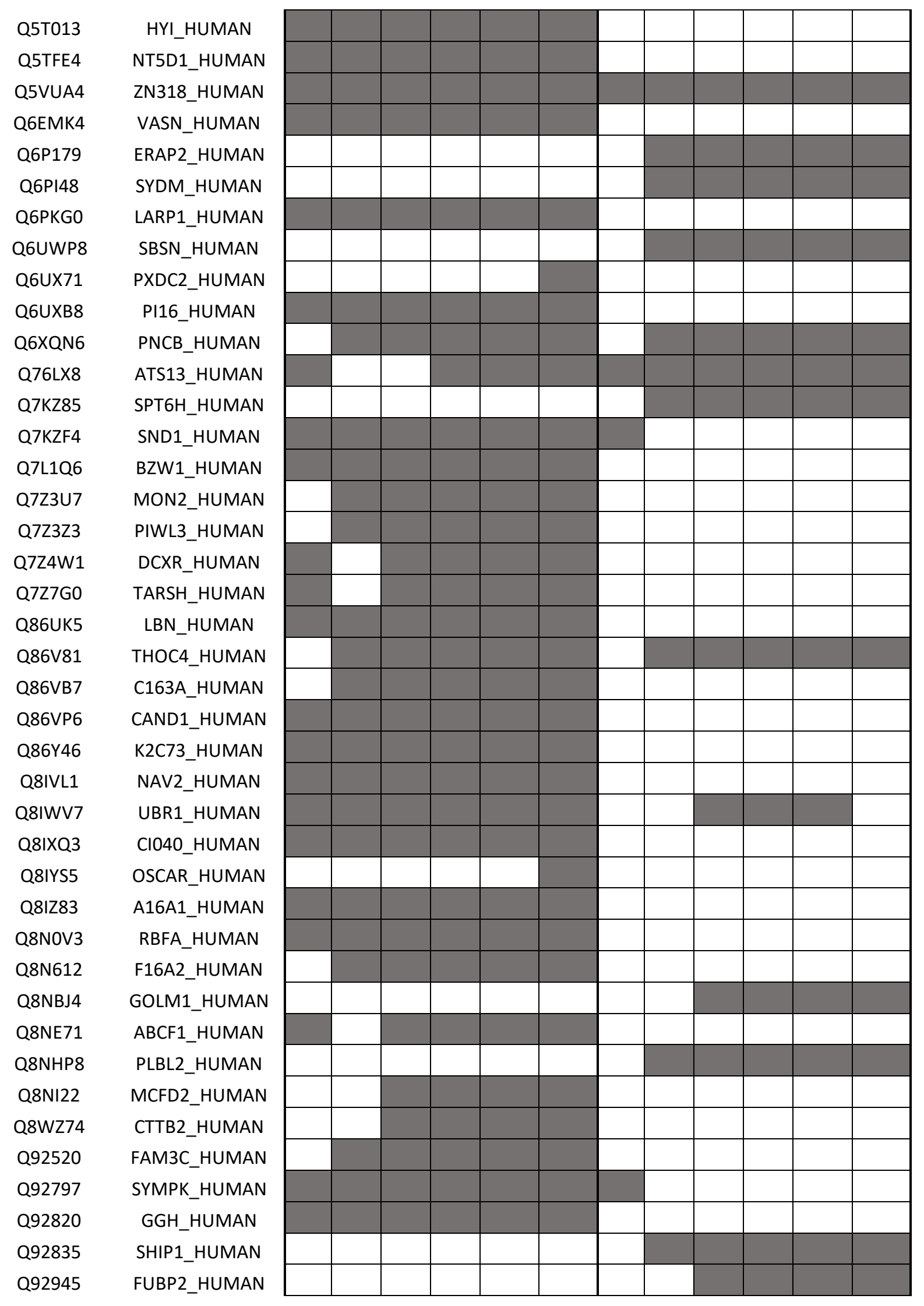




\begin{tabular}{|c|c|}
\hline Q92954 & PRG4_HUMAN \\
\hline Q93099 & HGD_HUMAN \\
\hline Q96BZ4 & PLD4_HUMAN \\
\hline Q96IU4 & ABHEB_HUMAN \\
\hline Q96IY4 & CBPB2_HUMAN \\
\hline Q96KN2 & CNDP1_HUMAN \\
\hline Q96PD5 & PGRP2_HUMAN \\
\hline Q96PK6 & RBM14_HUMAN \\
\hline Q99460 & PSMD1_HUMAN \\
\hline Q99497 & PARK7_HUMAN \\
\hline Q99683 & M3K5_HUMAN \\
\hline Q99729 & ROAA_HUMAN \\
\hline Q99784 & NOE1_HUMAN \\
\hline Q99829 & CPNE1_HUMAN \\
\hline Q99832 & TCPH_HUMAN \\
\hline Q99969 & RARR2_HUMAN \\
\hline Q9BQE5 & APOL2_HUMAN \\
\hline Q9BR76 & COR1B_HUMAN \\
\hline Q9BXR6 & FHR5_HUMAN \\
\hline Q9BY67 & CADM1_HUMAN \\
\hline Q9HOW9 & CK054_HUMAN \\
\hline Q9H4A4 & AMPB_HUMAN \\
\hline Q9H4M9 & EHD1_HUMAN \\
\hline Q9HAB8 & PPCS_HUMAN \\
\hline Q9HDC9 & APMAP_HUMAN \\
\hline Q9NNW7 & TRXR2_HUMAN \\
\hline Q9NQ79 & CRAC1_HUMAN \\
\hline Q9NTJ3 & SMC4_HUMAN \\
\hline Q9NY33 & DPP3_HUMAN \\
\hline Q9NYL2 & MLTK_HUMAN \\
\hline Q9NYU2 & UGGG1_HUMAN \\
\hline Q9NZP8 & C1RL_HUMAN \\
\hline Q9P1Y5 & CAMP3_HUMAN \\
\hline Q9P260 & K1468_HUMAN \\
\hline Q9UBF2 & COPG2_HUMAN \\
\hline Q9UBR2 & CATZ_HUMAN \\
\hline Q9UGM5 & FETUB_HUMAN \\
\hline Q9UI26 & IPO11_HUMAN \\
\hline Q9UK55 & ZPI_HUMAN \\
\hline Q9UKX7 & NUP50_HUMAN \\
\hline Q9UNN8 & EPCR_HUMAN \\
\hline
\end{tabular}

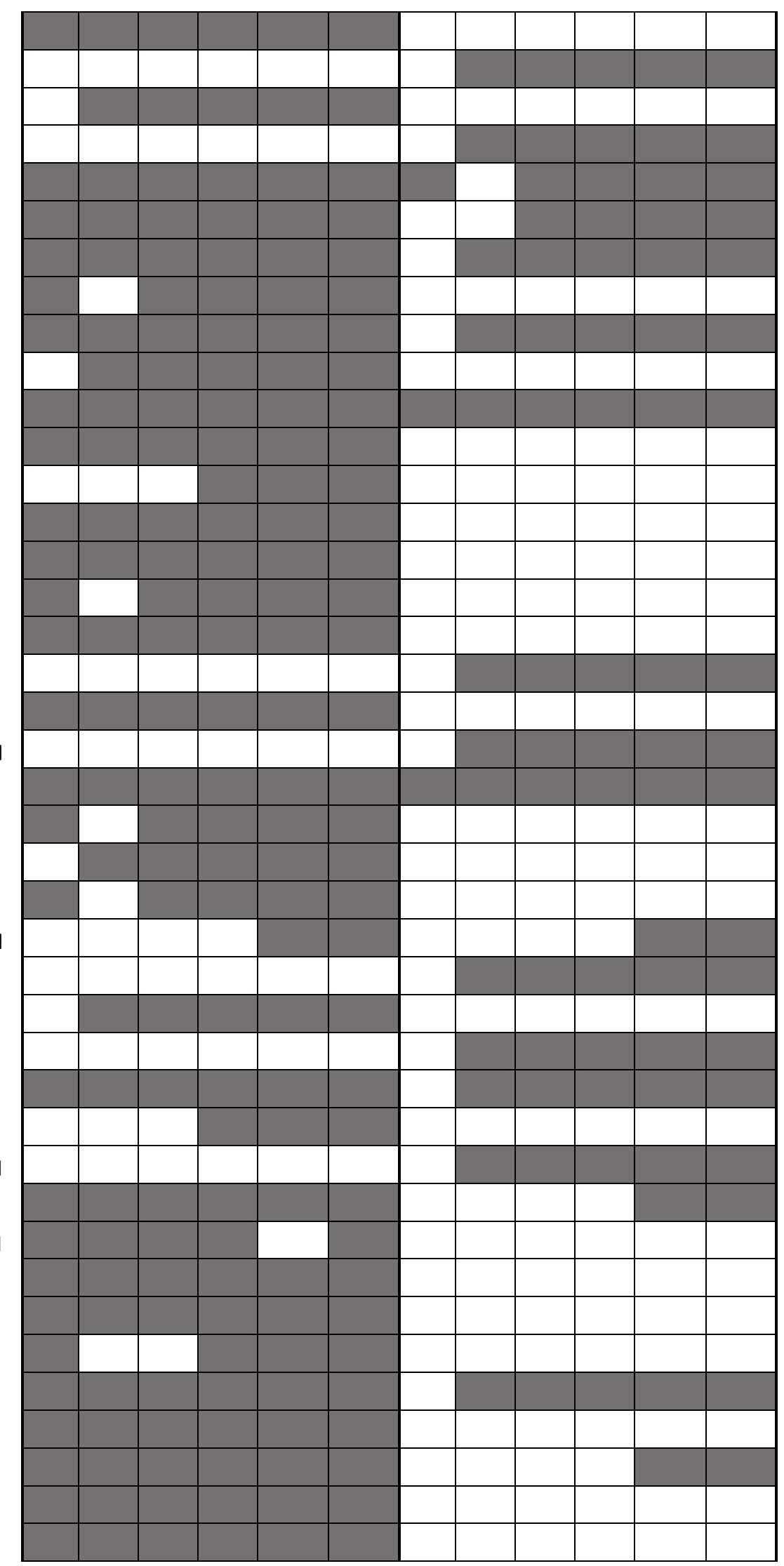




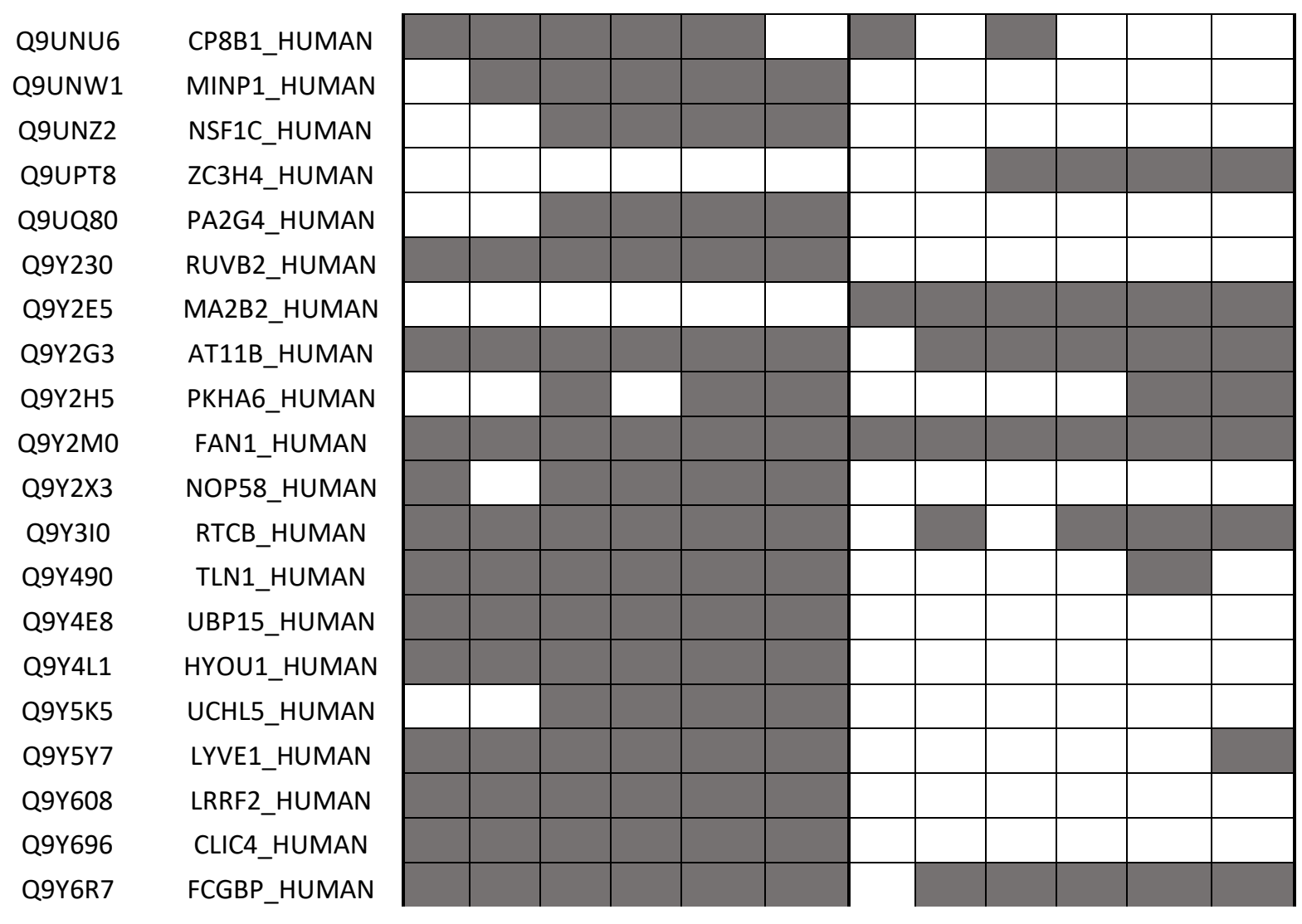


Supplementary table 4. Summary of the 757 proteins detected with increasing analytical stringency in this dual workflow approach. Proteins that are only identified are labeled "not quantifiable"; proteins without adequate linearity are labeled "N/A" in their linear range; proteins that are quantifiable from both depleted- and undepleted- plasma matrices are listed twice.

\begin{tabular}{|c|c|c|c|c|c|c|c|c|}
\hline$\underset{\text { ID }}{\text { Uniprot }}$ & Protein Name & $\begin{array}{c}\text { LLOQ } \\
\text { (ng plasma } \\
\text { on column) }\end{array}$ & $\begin{array}{c}\text { ULOQ } \\
\text { (ng plasma } \\
\text { on column) }\end{array}$ & Rsq & Intercept & Slope & $\begin{array}{l}\text { Linear } \\
\text { range }\end{array}$ & $\begin{array}{l}\text { Plasma } \\
\text { type }\end{array}$ \\
\hline A5YKK6 & CNOT1_HUMAN & $\begin{array}{c}\text { not } \\
\text { quantifiable }\end{array}$ & & & & & & Undepleted \\
\hline B9A064 & IGLL5_HUMAN & 63 & 2000 & 0.965 & 7.006 & 0.844 & $63-2000$ & Undepleted \\
\hline B9A064 & IGLL5_HUMAN & 63 & 1000 & 0.879 & 1.059 & 1.135 & $63-1000$ & Depleted \\
\hline 000187 & MASP2_HUMAN & 63 & 2000 & 0.961 & 2.716 & 0.948 & $63-2000$ & Depleted \\
\hline 000233 & PSMD9_HUMAN & $\begin{array}{c}\text { not } \\
\text { quantifiable }\end{array}$ & & & & & & Undepleted \\
\hline O00299 & CLIC1_HUMAN & 2000 & 2000 & 0.967 & -2.282 & 1.478 & $\mathrm{~N} / \mathrm{A}$ & Depleted \\
\hline O00391 & QSOX1_HUMAN & 63 & 2000 & 0.962 & 2.967 & 1.183 & $63-2000$ & Depleted \\
\hline 000410 & IPO5_HUMAN & 500 & 2000 & 0.842 & 4.714 & 0.734 & $500-2000$ & Depleted \\
\hline 000429 & DNM1L_HUMAN & 250 & 2000 & 0.365 & 4.722 & 0.846 & $250-2000$ & Undepleted \\
\hline 000429 & DNM1L_HUMAN & 125 & 2000 & 0.919 & 2.724 & 1.08 & $125-2000$ & Depleted \\
\hline O00468 & AGRIN_HUMAN & $\begin{array}{c}\text { not } \\
\text { quantifiable }\end{array}$ & & & & & & Undepleted \\
\hline 000533 & CHL1_HUMAN & 125 & 2000 & 0.957 & -0.341 & 1.465 & $125-2000$ & Depleted \\
\hline O00592 & PODXL_HUMAN & $\begin{array}{c}\text { not } \\
\text { quantifiable }\end{array}$ & & & & & & Depleted \\
\hline 014618 & CCS_HUMAN & 250 & 2000 & 0.795 & 5.33 & 0.769 & $250-2000$ & Depleted \\
\hline 014773 & TPP1_HUMAN & $\begin{array}{c}\text { not } \\
\text { quantifiable }\end{array}$ & & & & & & Depleted \\
\hline 014776 & TCRG1_HUMAN & 125 & 2000 & 0.958 & 5.327 & 0.876 & $125-2000$ & Undepleted \\
\hline 014791 & APOL1_HUMAN & 125 & 2000 & 0.964 & 3.6 & 0.981 & $125-2000$ & Undepleted \\
\hline 014791 & APOL1_HUMAN & 1000 & 2000 & 0.882 & -1.82 & 1.47 & $1000-2000$ & Depleted \\
\hline O14818 & PSA7_HUMAN & $\begin{array}{c}\text { not } \\
\text { quantifiable }\end{array}$ & & & & & & Depleted \\
\hline 014980 & XPO1_HUMAN & 63 & 2000 & 0.796 & 4.719 & 1.075 & $63-2000$ & Undepleted \\
\hline 014980 & XPO1_HUMAN & 2000 & 2000 & 0.889 & 5.987 & 0.484 & $\mathrm{~N} / \mathrm{A}$ & Depleted \\
\hline 015031 & PLXB2_HUMAN & $\begin{array}{c}\text { not } \\
\text { quantifiable }\end{array}$ & & & & & & Both \\
\hline 015067 & PUR4_HUMAN & 250 & 2000 & 0.969 & 4.584 & 0.767 & $250-2000$ & Undepleted \\
\hline 015067 & PUR4_HUMAN & 250 & 2000 & 0.916 & 5.505 & 0.765 & $250-2000$ & Depleted \\
\hline 015145 & ARPC3_HUMAN & $\begin{array}{c}\text { not } \\
\text { quantifiable }\end{array}$ & & & & & & Depleted \\
\hline 015212 & PFD6_HUMAN & $\begin{array}{c}\text { not } \\
\text { quantifiable }\end{array}$ & & & & & & Undepleted \\
\hline 015230 & LAMA5_HUMAN & 250 & 2000 & 0.889 & 5.004 & 0.76 & $250-2000$ & Depleted \\
\hline 015240 & VGF_HUMAN & $\begin{array}{c}\text { not } \\
\text { quantifiable }\end{array}$ & & & & & & Depleted \\
\hline 015269 & SPTC1_HUMAN & 125 & 2000 & 0.977 & 4.283 & 0.85 & $125-2000$ & Undepleted \\
\hline 015269 & SPTC1_HUMAN & 500 & 1000 & 0.924 & 7.149 & 0.497 & $500-1000$ & Depleted \\
\hline 015347 & HMGB3_HUMAN & 125 & 500 & 0.923 & 5.323 & 0.582 & $125-500$ & Undepleted \\
\hline 015400 & STX7_HUMAN & 250 & 2000 & 0.824 & 3.802 & 0.773 & $250-2000$ & Depleted \\
\hline
\end{tabular}




\begin{tabular}{|c|c|c|c|c|c|c|c|c|}
\hline O15523 & DDX3Y_HUMAN & 250 & 2000 & 0.891 & 5.133 & 0.802 & $250-2000$ & Depleted \\
\hline O43175 & SERA_HUMAN & 250 & 2000 & 0.802 & 5.127 & 0.844 & $250-2000$ & Depleted \\
\hline O43396 & TXNL1_HUMAN & 125 & 2000 & 0.863 & 5.156 & 0.917 & $125-2000$ & Undepleted \\
\hline O43505 & B3GN1_HUMAN & 125 & 2000 & 0.928 & 7.411 & 0.858 & $125-2000$ & Undepleted \\
\hline O43583 & DENR_HUMAN & 125 & 2000 & 0.918 & 4.791 & 0.944 & $125-2000$ & Undepleted \\
\hline O43598 & DNPH1_HUMAN & 500 & 2000 & 0.868 & 4.579 & 0.811 & $500-2000$ & Depleted \\
\hline O43707 & ACTN4_HUMAN & $\begin{array}{c}\text { not } \\
\text { quantifiable }\end{array}$ & & & & & & Undepleted \\
\hline O43776 & SYNC_HUMAN & 125 & 2000 & 0.947 & 5.27 & 0.934 & $125-2000$ & Undepleted \\
\hline 043776 & SYNC_HUMAN & 63 & 2000 & 0.967 & 5.01 & 0.858 & $63-2000$ & Depleted \\
\hline O43866 & CD5L_HUMAN & 63 & 2000 & 0.955 & 2.713 & 1.218 & $63-2000$ & Undepleted \\
\hline O43866 & CD5L_HUMAN & 250 & 2000 & 0.982 & -0.006 & 1.243 & $250-2000$ & Depleted \\
\hline O60306 & AQR_HUMAN & 125 & 2000 & 0.986 & 5.848 & 0.987 & $125-2000$ & Depleted \\
\hline 060610 & DIAP1_HUMAN & 125 & 2000 & 0.879 & 3.425 & 1.142 & $125-2000$ & Undepleted \\
\hline O60610 & DIAP1_HUMAN & 63 & 2000 & 0.92 & 4.364 & 0.997 & $63-2000$ & Depleted \\
\hline O60664 & PLIN3_HUMAN & 63 & 2000 & 0.943 & 4.308 & 1.01 & $63-2000$ & Depleted \\
\hline O60716 & CTND1_HUMAN & 500 & 2000 & 0.961 & 3.834 & 0.882 & $500-2000$ & Undepleted \\
\hline 075083 & WDR1_HUMAN & 125 & 2000 & 0.982 & 4.071 & 0.916 & $125-2000$ & Undepleted \\
\hline O75083 & WDR1_HUMAN & 63 & 2000 & 0.935 & 4.639 & 0.903 & $63-2000$ & Depleted \\
\hline O75131 & CPNE3_HUMAN & 1000 & 2000 & 0.981 & 2.49 & 0.892 & $1000-2000$ & Depleted \\
\hline O75144 & ICOSL_HUMAN & 250 & 2000 & 0.965 & 2.482 & 0.853 & $250-2000$ & Depleted \\
\hline O75368 & SH3L1_HUMAN & $\begin{array}{c}\text { not } \\
\text { quantifiable }\end{array}$ & & & & & & Undepleted \\
\hline O75369 & FLNB_HUMAN & 250 & 2000 & 0.863 & 3.601 & 1.096 & $250-2000$ & Depleted \\
\hline 075376 & NCOR1_HUMAN & 63 & 2000 & 0.976 & 6.414 & 1.029 & $63-2000$ & Undepleted \\
\hline O75380 & NDUS6_HUMAN & 125 & 2000 & 0.946 & 4.765 & 0.774 & $125-2000$ & Depleted \\
\hline 075390 & CISY_HUMAN & 63 & 2000 & 0.853 & 6.164 & 0.737 & $63-2000$ & Depleted \\
\hline O75636 & FCN3_HUMAN & 63 & 2000 & 0.935 & 2.206 & 1.086 & $63-2000$ & Undepleted \\
\hline O75636 & FCN3_HUMAN & 63 & 2000 & 0.981 & 5.485 & 1.017 & $63-2000$ & Depleted \\
\hline O75822 & EIF3J_HUMAN & 500 & 500 & 0.274 & 5.353 & 1.021 & $N / A$ & Undepleted \\
\hline O75822 & EIF3J_HUMAN & 500 & 2000 & 0.966 & 5.233 & 0.765 & $500-2000$ & Depleted \\
\hline O75874 & IDHC_HUMAN & $\begin{array}{c}\text { not } \\
\text { quantifiable }\end{array}$ & & & & & & Undepleted \\
\hline O75882 & ATRN_HUMAN & 250 & 2000 & 0.85 & -0.034 & 1.346 & $250-2000$ & Undepleted \\
\hline O75882 & ATRN_HUMAN & 63 & 2000 & 0.988 & 3.737 & 1.165 & $63-2000$ & Depleted \\
\hline 094776 & MTA2_HUMAN & 125 & 2000 & 0.981 & 5.795 & 0.786 & $125-2000$ & Undepleted \\
\hline O94804 & STK10_HUMAN & 250 & 2000 & 0.877 & 5.25 & 0.792 & $250-2000$ & Depleted \\
\hline O94919 & ENDD1_HUMAN & 250 & 2000 & 0.837 & 4.839 & 0.824 & $250-2000$ & Depleted \\
\hline 095336 & 6PGL_HUMAN & 63 & 2000 & 0.889 & 2.964 & 1.117 & $63-2000$ & Depleted \\
\hline 095373 & IPO7_HUMAN & 63 & 2000 & 0.9 & 3.974 & 1.018 & $63-2000$ & Depleted \\
\hline O95445 & APOM_HUMAN & 63 & 2000 & 0.949 & 3.127 & 1.027 & $63-2000$ & Undepleted \\
\hline 095445 & APOM_HUMAN & 1000 & 2000 & 0.895 & 0.921 & 1.023 & $1000-2000$ & Depleted \\
\hline O95479 & G6PE_HUMAN & $\begin{array}{c}\text { not } \\
\text { quantifiable }\end{array}$ & & & & & & Depleted \\
\hline O95497 & VNN1_HUMAN & 1000 & 2000 & 0.776 & -3.848 & 1.589 & $1000-2000$ & Depleted \\
\hline 095831 & AIFM1_HUMAN & 250 & 1000 & 0.38 & 8.143 & 0.348 & $250-1000$ & Depleted \\
\hline
\end{tabular}




\begin{tabular}{|c|c|c|c|c|c|c|c|c|}
\hline P00325 & ADH1B_HUMAN & 500 & 2000 & 0.541 & 4.71 & 0.788 & $500-2000$ & Depleted \\
\hline P00338 & LDHA_HUMAN & 250 & 2000 & 0.712 & 3.043 & 1.21 & $250-2000$ & Depleted \\
\hline P00352 & AL1A1_HUMAN & 250 & 2000 & 0.929 & 5.318 & 0.768 & $250-2000$ & Depleted \\
\hline P00367 & DHE3_HUMAN & 63 & 2000 & 0.923 & 4.447 & 0.986 & $63-2000$ & Undepleted \\
\hline P00450 & CERU_HUMAN & 63 & 2000 & 0.986 & 5.689 & 1.052 & $63-2000$ & Undepleted \\
\hline P00450 & CERU_HUMAN & 63 & 2000 & 0.973 & 10.18 & 0.936 & $63-2000$ & Depleted \\
\hline P00488 & F13A_HUMAN & 125 & 2000 & 0.949 & 0.439 & 1.537 & $125-2000$ & Depleted \\
\hline P00491 & PNPH_HUMAN & 63 & 2000 & 0.93 & 3.881 & 0.8 & $63-2000$ & Depleted \\
\hline P00505 & AATM_HUMAN & 125 & 2000 & 0.795 & 4.486 & 0.788 & $125-2000$ & Depleted \\
\hline P00533 & EGFR_HUMAN & 250 & 2000 & 0.935 & 2.648 & 1.066 & $250-2000$ & Undepleted \\
\hline P00558 & PGK1_HUMAN & 125 & 2000 & 0.924 & 4.277 & 0.85 & $125-2000$ & Undepleted \\
\hline P00734 & THRB_HUMAN & 63 & 2000 & 0.97 & 4.798 & 1.096 & $63-2000$ & Undepleted \\
\hline P00734 & THRB_HUMAN & 63 & 2000 & 0.973 & 8.755 & 0.966 & $63-2000$ & Depleted \\
\hline P00736 & C1R_HUMAN & 63 & 2000 & 0.98 & 2.618 & 1.152 & $63-2000$ & Undepleted \\
\hline P00736 & C1R_HUMAN & 63 & 2000 & 0.974 & 6.595 & 1.011 & $63-2000$ & Depleted \\
\hline P00739 & HPTR_HUMAN & 63 & 2000 & 0.98 & 7.688 & 0.907 & $63-2000$ & Undepleted \\
\hline P00739 & HPTR_HUMAN & 1000 & 2000 & 0.997 & 0.251 & 1.172 & $1000-2000$ & Depleted \\
\hline P00740 & FA9_HUMAN & 63 & 2000 & 0.98 & 5.871 & 0.942 & $63-2000$ & Depleted \\
\hline P00742 & FA10_HUMAN & 500 & 2000 & 0.783 & 1.158 & 1.082 & $500-2000$ & Undepleted \\
\hline P00742 & FA10_HUMAN & 63 & 2000 & 0.994 & 6.154 & 0.982 & $63-2000$ & Depleted \\
\hline P00746 & CFAD_HUMAN & 63 & 2000 & 0.974 & 4.153 & 1.067 & $63-2000$ & Depleted \\
\hline P00747 & PLMN_HUMAN & 63 & 2000 & 0.982 & 6.729 & 0.997 & $63-2000$ & Undepleted \\
\hline P00747 & PLMN_HUMAN & 63 & 2000 & 0.991 & 9.168 & 0.936 & $63-2000$ & Depleted \\
\hline P00748 & FA12_HUMAN & 63 & 2000 & 0.956 & 2.938 & 1.143 & $63-2000$ & Undepleted \\
\hline P00748 & FA12_HUMAN & 63 & 2000 & 0.977 & 7.135 & 0.974 & $63-2000$ & Depleted \\
\hline P00751 & CFAB_HUMAN & 63 & 2000 & 0.975 & 6.591 & 0.995 & $63-2000$ & Undepleted \\
\hline P00751 & CFAB_HUMAN & 63 & 2000 & 0.984 & 9.606 & 0.933 & $63-2000$ & Depleted \\
\hline P00813 & ADA_HUMAN & 250 & 2000 & 0.853 & 5.966 & 0.546 & $250-2000$ & Depleted \\
\hline P00915 & CAH1_HUMAN & 250 & 2000 & 0.944 & 1.043 & 1.152 & $250-2000$ & Depleted \\
\hline P01008 & ANT3_HUMAN & 63 & 2000 & 0.983 & 5.229 & 1.064 & $63-2000$ & Undepleted \\
\hline P01008 & ANT3_HUMAN & 63 & 2000 & 0.986 & 9.512 & 0.924 & $63-2000$ & Depleted \\
\hline P01011 & AACT_HUMAN & 63 & 2000 & 0.985 & 6.921 & 1.027 & $63-2000$ & Undepleted \\
\hline P01011 & AACT_HUMAN & 63 & 2000 & 0.97 & 10.125 & 0.846 & $63-2000$ & Depleted \\
\hline P01019 & ANGT_HUMAN & 63 & 2000 & 0.987 & 5.571 & 0.932 & $63-2000$ & Undepleted \\
\hline P01019 & ANGT_HUMAN & 63 & 2000 & 0.979 & 8.215 & 0.894 & $63-2000$ & Depleted \\
\hline P01031 & CO5_HUMAN & 63 & 2000 & 0.965 & 2.239 & 1.438 & $63-2000$ & Undepleted \\
\hline P01031 & CO5_HUMAN & 63 & 2000 & 0.98 & 7.468 & 1.021 & $63-2000$ & Depleted \\
\hline P01034 & CYTC_HUMAN & 63 & 2000 & 0.97 & 2.355 & 1.167 & $63-2000$ & Depleted \\
\hline P01042 & KNG1_HUMAN & 63 & 2000 & 0.985 & 6.475 & 0.975 & $63-2000$ & Undepleted \\
\hline P01042 & KNG1_HUMAN & 63 & 2000 & 0.97 & 9.367 & 0.872 & $63-2000$ & Depleted \\
\hline P01130 & LDLR_HUMAN & $\begin{array}{c}\text { not } \\
\text { quantifiable }\end{array}$ & & & & & & Depleted \\
\hline P01344 & IGF2_HUMAN & 63 & 2000 & 0.954 & 3.335 & 1.066 & $63-2000$ & Depleted \\
\hline
\end{tabular}




\begin{tabular}{|c|c|c|c|c|c|c|c|c|}
\hline P01591 & IGJ_HUMAN & 63 & 2000 & 0.887 & 3.776 & 1.218 & $63-2000$ & Undepleted \\
\hline P01591 & IGJ_HUMAN & 125 & 2000 & 0.886 & 0.408 & 1.299 & $125-2000$ & Depleted \\
\hline P01593 & KV101_HUMAN & 125 & 2000 & 0.936 & 2.882 & 0.97 & $125-2000$ & Undepleted \\
\hline P01594 & KV102_HUMAN & 63 & 2000 & 0.981 & 2.952 & 0.933 & $63-2000$ & Undepleted \\
\hline P01597 & KV105_HUMAN & 250 & 1000 & 0.915 & 3.123 & 0.773 & $250-1000$ & Undepleted \\
\hline P01608 & KV116_HUMAN & 63 & 2000 & 0.977 & 3.52 & 0.986 & $63-2000$ & Undepleted \\
\hline P01611 & KV119_HUMAN & $\begin{array}{c}\text { not } \\
\text { quantifiable }\end{array}$ & & & & & & Undepleted \\
\hline P01621 & KV303_HUMAN & 63 & 2000 & 0.907 & 2.11 & 1.361 & $63-2000$ & Undepleted \\
\hline P01621 & KV303_HUMAN & 500 & 2000 & 0.83 & 1.954 & 0.957 & $500-2000$ & Depleted \\
\hline P01714 & LV301_HUMAN & 63 & 2000 & 0.95 & 2.484 & 1.192 & $63-2000$ & Undepleted \\
\hline P01717 & LV403_HUMAN & 63 & 2000 & 0.983 & 3.072 & 1.07 & $63-2000$ & Undepleted \\
\hline P01764 & HV303_HUMAN & 1000 & 2000 & 0.958 & 1.781 & 0.873 & $1000-2000$ & Depleted \\
\hline P01780 & HV319_HUMAN & 63 & 2000 & 0.984 & 4.351 & 1.056 & $63-2000$ & Undepleted \\
\hline P01781 & HV320_HUMAN & 63 & 2000 & 0.99 & 4.161 & 0.936 & $63-2000$ & Undepleted \\
\hline P01824 & HV206_HUMAN & $\begin{array}{c}\text { not } \\
\text { quantifiable }\end{array}$ & & & & & & Undepleted \\
\hline P01834 & IGKC_HUMAN & 63 & 2000 & 0.988 & 8.542 & 0.931 & $63-2000$ & Undepleted \\
\hline P01834 & IGKC_HUMAN & 63 & 2000 & 0.945 & 2.753 & 1.256 & $63-2000$ & Depleted \\
\hline P01861 & IGHG4_HUMAN & 63 & 2000 & 0.949 & 4.345 & 1.082 & $63-2000$ & Undepleted \\
\hline P01880 & IGHD_HUMAN & 63 & 2000 & 0.958 & 2.519 & 0.96 & $63-2000$ & Undepleted \\
\hline P02042 & HBD_HUMAN & 250 & 2000 & 0.806 & 6.214 & 0.892 & $250-2000$ & Undepleted \\
\hline P02461 & CO3A1_HUMAN & 125 & 2000 & 0.974 & 4.618 & 0.872 & $125-2000$ & Undepleted \\
\hline P02461 & CO3A1_HUMAN & 500 & 1000 & 0.885 & 5.875 & 0.565 & $500-1000$ & Depleted \\
\hline P02533 & K1C14_HUMAN & $\begin{array}{c}\text { not } \\
\text { quantifiable }\end{array}$ & & & & & & Undepleted \\
\hline P02545 & LMNA_HUMAN & 63 & 2000 & 0.501 & 8.792 & 0.363 & $63-2000$ & Depleted \\
\hline P02649 & APOE_HUMAN & 63 & 2000 & 0.984 & 5.567 & 1.031 & $63-2000$ & Undepleted \\
\hline P02649 & APOE_HUMAN & 63 & 2000 & 0.992 & 5.182 & 1.099 & $63-2000$ & Depleted \\
\hline P02654 & APOC1_HUMAN & 63 & 2000 & 0.985 & 6.323 & 0.98 & $63-2000$ & Undepleted \\
\hline P02654 & APOC1_HUMAN & 1000 & 2000 & 0.988 & -2.714 & 1.593 & $1000-2000$ & Depleted \\
\hline P02655 & APOC2_HUMAN & 63 & 2000 & 0.983 & 5.15 & 0.987 & $63-2000$ & Undepleted \\
\hline P02655 & APOC2_HUMAN & 63 & 2000 & 0.908 & 2.421 & 1.16 & $63-2000$ & Depleted \\
\hline P02656 & APOC3_HUMAN & 63 & 2000 & 0.973 & 6.174 & 0.987 & $63-2000$ & Undepleted \\
\hline P02656 & APOC3_HUMAN & 63 & 2000 & 0.981 & 4.917 & 1.038 & $63-2000$ & Depleted \\
\hline P02730 & B3AT_HUMAN & 500 & 2000 & 0.904 & 4.328 & 0.802 & $500-2000$ & Depleted \\
\hline P02741 & CRP_HUMAN & 63 & 2000 & 0.963 & 4.501 & 1.05 & $63-2000$ & Depleted \\
\hline P02743 & SAMP_HUMAN & 1000 & 2000 & 0.986 & -1.084 & 1.357 & $1000-2000$ & Undepleted \\
\hline P02743 & SAMP_HUMAN & 63 & 2000 & 0.992 & 7.503 & 0.929 & $63-2000$ & Depleted \\
\hline P02745 & C1QA_HUMAN & 500 & 2000 & 0.989 & 3.491 & 0.885 & $500-2000$ & Undepleted \\
\hline P02745 & C1QA_HUMAN & 63 & 2000 & 0.973 & 5.845 & 0.976 & $63-2000$ & Depleted \\
\hline P02746 & C1QB_HUMAN & 125 & 2000 & 0.976 & 3.45 & 1.029 & $125-2000$ & Undepleted \\
\hline P02746 & C1QB_HUMAN & 63 & 2000 & 0.96 & 7.281 & 0.907 & $63-2000$ & Depleted \\
\hline P02747 & C1QC_HUMAN & 63 & 2000 & 0.961 & 2.39 & 1.094 & $63-2000$ & Undepleted \\
\hline P02747 & C1QC_HUMAN & 63 & 2000 & 0.996 & 7.17 & 0.948 & $63-2000$ & Depleted \\
\hline
\end{tabular}




\begin{tabular}{|c|c|c|c|c|c|c|c|c|}
\hline P02748 & CO9_HUMAN & 63 & 2000 & 0.96 & 2.669 & 1.237 & $63-2000$ & Undepleted \\
\hline P02748 & CO9_HUMAN & 63 & 2000 & 0.991 & 7.75 & 0.945 & $63-2000$ & Depleted \\
\hline P02749 & APOH_HUMAN & 63 & 2000 & 0.706 & 7.345 & 0.659 & $63-2000$ & Undepleted \\
\hline P02749 & APOH_HUMAN & 63 & 2000 & 0.984 & 8.795 & 0.968 & $63-2000$ & Depleted \\
\hline P02750 & A2GL_HUMAN & 63 & 2000 & 0.969 & 2.953 & 1.171 & $63-2000$ & Undepleted \\
\hline P02750 & A2GL_HUMAN & 63 & 2000 & 0.988 & 7.52 & 0.963 & $63-2000$ & Depleted \\
\hline P02751 & FINC_HUMAN & 63 & 2000 & 0.961 & 3.94 & 1.182 & $63-2000$ & Undepleted \\
\hline P02751 & FINC_HUMAN & 63 & 2000 & 0.987 & 8.548 & 0.991 & $63-2000$ & Depleted \\
\hline P02753 & RET4_HUMAN & 63 & 2000 & 0.776 & 3.952 & 0.8 & $63-2000$ & Undepleted \\
\hline P02753 & RET4_HUMAN & 63 & 2000 & 0.985 & 8.094 & 0.93 & $63-2000$ & Depleted \\
\hline P02760 & AMBP_HUMAN & 63 & 2000 & 0.987 & 4.988 & 1.009 & $63-2000$ & Undepleted \\
\hline P02760 & AMBP_HUMAN & 63 & 2000 & 0.985 & 8.263 & 0.944 & $63-2000$ & Depleted \\
\hline P02765 & FETUA_HUMAN & 63 & 2000 & 0.983 & 6.373 & 0.995 & $63-2000$ & Undepleted \\
\hline P02765 & FETUA_HUMAN & 63 & 2000 & 0.987 & 9.307 & 1.001 & $63-2000$ & Depleted \\
\hline P02766 & TTHY_HUMAN & 63 & 2000 & 0.971 & 9.096 & 0.971 & $63-2000$ & Depleted \\
\hline P02774 & VTDB_HUMAN & 125 & 2000 & 0.922 & 5.706 & 1.144 & $125-2000$ & Undepleted \\
\hline P02774 & VTDB_HUMAN & 63 & 2000 & 0.961 & 9.76 & 0.94 & $63-2000$ & Depleted \\
\hline P02775 & CXCL7_HUMAN & 250 & 2000 & 0.859 & 2.171 & 0.996 & $250-2000$ & Undepleted \\
\hline P02775 & CXCL7_HUMAN & 125 & 2000 & 0.924 & 1.023 & 1.315 & $125-2000$ & Depleted \\
\hline P02776 & PLF4_HUMAN & $\begin{array}{c}\text { not } \\
\text { quantifiable }\end{array}$ & & & & & & Undepleted \\
\hline P02788 & TRFL_HUMAN & $\begin{array}{c}\text { not } \\
\text { quantifiable }\end{array}$ & & & & & & Undepleted \\
\hline P02790 & HEMO_HUMAN & 63 & 2000 & 0.981 & 7.441 & 1.068 & $63-2000$ & Undepleted \\
\hline P02790 & HEMO_HUMAN & 63 & 2000 & 0.981 & 11.141 & 0.913 & $63-2000$ & Depleted \\
\hline P02794 & FRIH_HUMAN & 63 & 2000 & 0.979 & 5.184 & 0.927 & $63-2000$ & Depleted \\
\hline P03951 & FA11_HUMAN & 2000 & 2000 & 0.999 & 2.451 & 0.816 & $N / A$ & Undepleted \\
\hline P03951 & FA11_HUMAN & 63 & 2000 & 0.97 & 3.258 & 1.249 & $63-2000$ & Depleted \\
\hline P03952 & KLKB1_HUMAN & 63 & 2000 & 0.796 & 1.943 & 1.411 & $63-2000$ & Undepleted \\
\hline P03952 & KLKB1_HUMAN & 63 & 2000 & 0.991 & 5.693 & 1.051 & $63-2000$ & Depleted \\
\hline P04003 & C4BPA_HUMAN & 63 & 2000 & 0.975 & 5.789 & 1.038 & $63-2000$ & Undepleted \\
\hline P04003 & C4BPA_HUMAN & 63 & 2000 & 0.967 & 9.422 & 0.857 & $63-2000$ & Depleted \\
\hline P04004 & VTNC_HUMAN & 63 & 2000 & 0.978 & 6.969 & 0.924 & $63-2000$ & Undepleted \\
\hline P04004 & VTNC_HUMAN & 63 & 2000 & 0.991 & 8.938 & 0.907 & $63-2000$ & Depleted \\
\hline P04040 & CATA_HUMAN & 250 & 2000 & 0.89 & 6.501 & 0.578 & $250-2000$ & Depleted \\
\hline P04054 & PA21B_HUMAN & 250 & 2000 & 0.959 & 5.422 & 0.949 & $250-2000$ & Undepleted \\
\hline P04066 & FUCO_HUMAN & $\begin{array}{c}\text { not } \\
\text { quantifiable }\end{array}$ & & & & & & Depleted \\
\hline P04070 & PROC_HUMAN & 63 & 2000 & 0.986 & 4.116 & 1.122 & $63-2000$ & Depleted \\
\hline P04075 & ALDOA_HUMAN & 125 & 2000 & 0.987 & 6.089 & 0.806 & $125-2000$ & Undepleted \\
\hline P04083 & ANXA1_HUMAN & 500 & 2000 & 0.974 & 5.423 & 0.746 & $500-2000$ & Undepleted \\
\hline P04179 & SODM_HUMAN & 250 & 2000 & 0.95 & 4.279 & 0.759 & $250-2000$ & Undepleted \\
\hline P04179 & SODM_HUMAN & 63 & 2000 & 0.858 & 3.892 & 1.033 & $63-2000$ & Depleted \\
\hline P04180 & LCAT_HUMAN & 500 & 2000 & 0.958 & 2.722 & 0.789 & $500-2000$ & Undepleted \\
\hline P04180 & LCAT_HUMAN & 63 & 2000 & 0.964 & 3.627 & 1.08 & $63-2000$ & Depleted \\
\hline
\end{tabular}




\begin{tabular}{|c|c|c|c|c|c|c|c|c|}
\hline P04196 & HRG_HUMAN & 63 & 2000 & 0.959 & 5.454 & 1.014 & $63-2000$ & Undepleted \\
\hline P04196 & HRG_HUMAN & 63 & 2000 & 0.984 & 9.389 & 0.886 & $63-2000$ & Depleted \\
\hline P04207 & KV308_HUMAN & $\begin{array}{c}\text { not } \\
\text { quantifiable }\end{array}$ & & & & & & Undepleted \\
\hline P04211 & LV001_HUMAN & 63 & 2000 & 0.979 & 3.544 & 0.973 & $63-2000$ & Undepleted \\
\hline P04217 & A1BG_HUMAN & 63 & 2000 & 0.981 & 5.744 & 1.087 & $63-2000$ & Undepleted \\
\hline P04217 & A1BG_HUMAN & 63 & 2000 & 0.983 & 8.761 & 0.912 & $63-2000$ & Depleted \\
\hline P04220 & MUCB_HUMAN & 2000 & 2000 & 0.995 & 4.031 & 0.734 & $\mathrm{~N} / \mathrm{A}$ & Undepleted \\
\hline P04220 & MUCB_HUMAN & 125 & 2000 & 0.89 & 2.462 & 1.07 & $125-2000$ & Depleted \\
\hline P04264 & K2C1_HUMAN & 250 & 2000 & 0.851 & 5.624 & 0.741 & $250-2000$ & Depleted \\
\hline P04275 & VWF_HUMAN & 2000 & 2000 & 0.93 & -1.176 & 1.256 & $\mathrm{~N} / \mathrm{A}$ & Undepleted \\
\hline P04275 & VWF_HUMAN & 125 & 2000 & 0.976 & -0.077 & 1.588 & $125-2000$ & Depleted \\
\hline P04278 & SHBG_HUMAN & 1000 & 2000 & 0.857 & 2.298 & 0.966 & $1000-2000$ & Undepleted \\
\hline P04278 & SHBG_HUMAN & 63 & 2000 & 0.989 & 4.436 & 1.008 & $63-2000$ & Depleted \\
\hline P04406 & G3P_HUMAN & 125 & 2000 & 0.933 & 6.181 & 0.723 & $125-2000$ & Undepleted \\
\hline P04406 & G3P_HUMAN & 125 & 2000 & 0.826 & 4.263 & 0.96 & $125-2000$ & Depleted \\
\hline P04430 & KV122_HUMAN & 63 & 2000 & 0.966 & 3.271 & 1.034 & $63-2000$ & Undepleted \\
\hline P04433 & KV309_HUMAN & 63 & 2000 & 0.992 & 4.917 & 1.006 & $63-2000$ & Undepleted \\
\hline P04433 & KV309_HUMAN & 1000 & 2000 & 0.958 & 2.976 & 0.81 & $1000-2000$ & Depleted \\
\hline P04632 & CPNS1_HUMAN & $\begin{array}{c}\text { not } \\
\text { quantifiable }\end{array}$ & & & & & & Both \\
\hline P05019 & IGF1_HUMAN & 63 & 2000 & 0.974 & 0.859 & 1.21 & $63-2000$ & Depleted \\
\hline P05062 & ALDOB_HUMAN & 63 & 2000 & 0.888 & 3.955 & 1.145 & $63-2000$ & Undepleted \\
\hline P05062 & ALDOB_HUMAN & 63 & 2000 & 0.856 & 2.178 & 1.186 & $63-2000$ & Depleted \\
\hline P05067 & A4_HUMAN & 63 & 2000 & 0.971 & 4.428 & 0.871 & $63-2000$ & Undepleted \\
\hline P05090 & APOD_HUMAN & 63 & 2000 & 0.989 & 5.506 & 0.986 & $63-2000$ & Undepleted \\
\hline P05090 & APOD_HUMAN & 500 & 2000 & 0.975 & 2.221 & 1.038 & $500-2000$ & Depleted \\
\hline P05091 & ALDH2_HUMAN & 250 & 2000 & 0.964 & 6.683 & 0.738 & $250-2000$ & Undepleted \\
\hline P05091 & ALDH2_HUMAN & 63 & 2000 & 0.934 & 3.418 & 1.161 & $63-2000$ & Depleted \\
\hline P05109 & S10A8_HUMAN & 250 & 2000 & 0.938 & 0.351 & 1.372 & $250-2000$ & Depleted \\
\hline P05121 & PAI1_HUMAN & 500 & 2000 & 0.893 & 3.989 & 0.895 & $500-2000$ & Depleted \\
\hline P05154 & IPSP_HUMAN & $\begin{array}{c}\text { not } \\
\text { quantifiable }\end{array}$ & & & & & & Both \\
\hline P05155 & IC1_HUMAN & 63 & 2000 & 0.981 & 5.084 & 1.065 & $63-2000$ & Undepleted \\
\hline P05155 & IC1_HUMAN & 63 & 2000 & 0.987 & 8.528 & 0.878 & $63-2000$ & Depleted \\
\hline P05156 & CFAI_HUMAN & 63 & 2000 & 0.86 & 1.469 & 1.358 & $63-2000$ & Undepleted \\
\hline P05156 & CFAI_HUMAN & 63 & 2000 & 0.975 & 7.495 & 0.959 & $63-2000$ & Depleted \\
\hline P05160 & F13B_HUMAN & 1000 & 2000 & 0.969 & -12.885 & 2.981 & $1000-2000$ & Undepleted \\
\hline P05160 & F13B_HUMAN & 63 & 2000 & 0.965 & 1.833 & 1.312 & $63-2000$ & Depleted \\
\hline P05164 & PERM_HUMAN & 250 & 2000 & 0.752 & 7.704 & 0.645 & $250-2000$ & Undepleted \\
\hline P05165 & PCCA_HUMAN & 125 & 2000 & 0.978 & 5.264 & 0.829 & $125-2000$ & Undepleted \\
\hline P05165 & PCCA_HUMAN & 125 & 2000 & 0.917 & 6.786 & 0.709 & $125-2000$ & Depleted \\
\hline P05166 & PCCB_HUMAN & 250 & 2000 & 0.897 & 4.245 & 0.86 & $250-2000$ & Depleted \\
\hline P05186 & PPBT_HUMAN & 125 & 2000 & 0.941 & 6.332 & 0.754 & $125-2000$ & Depleted \\
\hline P05362 & ICAM1_HUMAN & 125 & 2000 & 0.955 & 3.425 & 0.851 & $125-2000$ & Depleted \\
\hline
\end{tabular}




\begin{tabular}{|c|c|c|c|c|c|c|c|c|}
\hline P05413 & FABPH_HUMAN & 250 & 2000 & 0.977 & 3.855 & 0.837 & $250-2000$ & Undepleted \\
\hline P05452 & TETN_HUMAN & 63 & 2000 & 0.957 & 2.939 & 0.855 & $63-2000$ & Undepleted \\
\hline P05452 & TETN_HUMAN & 63 & 2000 & 0.987 & 6.37 & 0.837 & $63-2000$ & Depleted \\
\hline P05543 & THBG_HUMAN & 63 & 2000 & 0.965 & 6.058 & 1.003 & $63-2000$ & Depleted \\
\hline P05546 & HEP2_HUMAN & 63 & 2000 & 0.953 & 4.451 & 1.15 & $63-2000$ & Undepleted \\
\hline P05546 & HEP2_HUMAN & 63 & 2000 & 0.965 & 9.115 & 0.818 & $63-2000$ & Depleted \\
\hline P05556 & ITB1_HUMAN & 125 & 2000 & 0.747 & 3.28 & 1.433 & $125-2000$ & Undepleted \\
\hline P05787 & K2C8_HUMAN & 125 & 2000 & 0.805 & 6.077 & 0.802 & $125-2000$ & Undepleted \\
\hline P06132 & DCUP_HUMAN & 250 & 2000 & 0.901 & 3.898 & 0.839 & $250-2000$ & Depleted \\
\hline P06276 & CHLE_HUMAN & 1000 & 2000 & 0.619 & 2.995 & 0.751 & $1000-2000$ & Undepleted \\
\hline P06276 & CHLE_HUMAN & 63 & 2000 & 0.978 & 5.232 & 1.024 & $63-2000$ & Depleted \\
\hline P06310 & KV206_HUMAN & 63 & 2000 & 0.976 & 3.793 & 0.921 & $63-2000$ & Undepleted \\
\hline P06331 & HV209_HUMAN & 63 & 2000 & 0.983 & 3.551 & 1 & $63-2000$ & Undepleted \\
\hline P06396 & GELS_HUMAN & 63 & 2000 & 0.965 & 4.968 & 0.994 & $63-2000$ & Undepleted \\
\hline P06396 & GELS_HUMAN & 63 & 2000 & 0.967 & 7.066 & 1.014 & $63-2000$ & Depleted \\
\hline P06576 & ATPB_HUMAN & 250 & 2000 & 0.979 & 5.28 & 0.855 & $250-2000$ & Undepleted \\
\hline P06576 & ATPB_HUMAN & 63 & 2000 & 0.894 & 0.643 & 1.499 & $63-2000$ & Depleted \\
\hline P06681 & CO2_HUMAN & 500 & 2000 & 0.949 & -0.279 & 1.402 & $500-2000$ & Undepleted \\
\hline P06681 & CO2_HUMAN & 63 & 2000 & 0.988 & 7.341 & 0.954 & $63-2000$ & Depleted \\
\hline P06702 & S10A9_HUMAN & 500 & 2000 & 0.965 & 0.611 & 1.233 & $500-2000$ & Depleted \\
\hline P06703 & S10A6_HUMAN & $\begin{array}{c}\text { not } \\
\text { quantifiable }\end{array}$ & & & & & & Depleted \\
\hline P06727 & APOA4_HUMAN & 63 & 2000 & 0.993 & 6.588 & 0.997 & $63-2000$ & Undepleted \\
\hline P06727 & APOA4_HUMAN & 63 & 2000 & 0.993 & 8.94 & 0.945 & $63-2000$ & Depleted \\
\hline P06733 & ENOA_HUMAN & 125 & 2000 & 0.927 & 4.6 & 0.98 & $125-2000$ & Undepleted \\
\hline P06737 & PYGL_HUMAN & 63 & 250 & 0.86 & 2.866 & 1.131 & $63-250$ & Undepleted \\
\hline P06744 & G6PI_HUMAN & 125 & 1000 & 0.283 & 5.286 & 0.69 & $125-1000$ & Undepleted \\
\hline P06744 & G6PI_HUMAN & 125 & 2000 & 0.923 & 4.89 & 0.793 & $125-2000$ & Depleted \\
\hline P06748 & NPM_HUMAN & 250 & 2000 & 0.985 & 3.367 & 0.979 & $250-2000$ & Depleted \\
\hline P06865 & HEXA_HUMAN & 250 & 2000 & 0.874 & 6.038 & 0.675 & $250-2000$ & Depleted \\
\hline P07195 & LDHB_HUMAN & 63 & 2000 & 0.866 & 1.656 & 1.251 & $63-2000$ & Depleted \\
\hline P07203 & GPX1_HUMAN & $\begin{array}{c}\text { not } \\
\text { quantifiable }\end{array}$ & & & & & & Both \\
\hline P07225 & PROS_HUMAN & 125 & 2000 & 0.946 & 1.455 & 1.244 & $125-2000$ & Undepleted \\
\hline P07225 & PROS_HUMAN & 63 & 2000 & 0.983 & 7.076 & 0.92 & $63-2000$ & Depleted \\
\hline P07237 & PDIA1_HUMAN & 63 & 2000 & 0.895 & 4.499 & 0.914 & $63-2000$ & Depleted \\
\hline P07333 & CSF1R_HUMAN & 250 & 2000 & 0.916 & 1.411 & 1.052 & $250-2000$ & Depleted \\
\hline P07339 & CATD_HUMAN & 1000 & 2000 & 0.802 & 0.592 & 1.09 & $1000-2000$ & Depleted \\
\hline P07355 & ANXA2_HUMAN & 63 & 2000 & 0.582 & 6.278 & 0.867 & $63-2000$ & Undepleted \\
\hline P07355 & ANXA2_HUMAN & 125 & 500 & 0.989 & 5.049 & 0.923 & $125-500$ & Depleted \\
\hline P07357 & CO8A_HUMAN & 63 & 2000 & 0.917 & 2.283 & 1.252 & $63-2000$ & Undepleted \\
\hline P07357 & CO8A_HUMAN & 63 & 2000 & 0.982 & 7.293 & 0.925 & $63-2000$ & Depleted \\
\hline P07358 & CO8B_HUMAN & 63 & 2000 & 0.959 & 1.626 & 1.304 & $63-2000$ & Undepleted \\
\hline P07358 & CO8B_HUMAN & 63 & 2000 & 0.982 & 6.978 & 1.001 & $63-2000$ & Depleted \\
\hline
\end{tabular}




\begin{tabular}{|c|c|c|c|c|c|c|c|c|}
\hline P07359 & GP1BA_HUMAN & 250 & 2000 & 0.899 & 1.705 & 1.062 & $250-2000$ & Depleted \\
\hline P07360 & CO8G_HUMAN & 63 & 2000 & 0.953 & 3.141 & 1.081 & $63-2000$ & Undepleted \\
\hline P07360 & CO8G_HUMAN & 63 & 2000 & 0.961 & 7.11 & 0.927 & $63-2000$ & Depleted \\
\hline P07384 & CAN1_HUMAN & $\begin{array}{c}\text { not } \\
\text { quantifiable }\end{array}$ & & & & & & Depleted \\
\hline P07686 & HEXB_HUMAN & 250 & 1000 & 0.467 & 4.209 & 0.76 & $250-1000$ & Depleted \\
\hline P07711 & CATL1_HUMAN & $\begin{array}{c}\text { not } \\
\text { quantifiable }\end{array}$ & & & & & & Undepleted \\
\hline P07814 & SYEP_HUMAN & $\begin{array}{c}\text { not } \\
\text { quantifiable }\end{array}$ & & & & & & Depleted \\
\hline P07900 & HS90A_HUMAN & 125 & 2000 & 0.937 & 6.417 & 0.711 & $125-2000$ & Undepleted \\
\hline P07942 & LAMB1_HUMAN & 63 & 2000 & 0.847 & 3.434 & 1.232 & $63-2000$ & Depleted \\
\hline P07954 & FUMH_HUMAN & 250 & 2000 & 0.884 & 4.916 & 0.874 & $250-2000$ & Depleted \\
\hline P07996 & TSP1_HUMAN & $\begin{array}{c}\text { not } \\
\text { quantifiable }\end{array}$ & & & & & & Both \\
\hline P07998 & RNAS1_HUMAN & 500 & 2000 & 0.997 & 1.285 & 1.071 & $500-2000$ & Depleted \\
\hline P08107 & HSP71_HUMAN & 250 & 2000 & 0.916 & 5.585 & 0.756 & $250-2000$ & Depleted \\
\hline P08133 & ANXA6_HUMAN & $\begin{array}{c}\text { not } \\
\text { quantifiable }\end{array}$ & & & & & & Depleted \\
\hline P08185 & CBG_HUMAN & 125 & 2000 & 0.915 & 2.759 & 1.11 & $125-2000$ & Undepleted \\
\hline P08185 & CBG_HUMAN & 63 & 2000 & 0.957 & 6.43 & 0.87 & $63-2000$ & Depleted \\
\hline P08195 & 4F2_HUMAN & 125 & 2000 & 0.979 & 5.333 & 0.777 & $125-2000$ & Undepleted \\
\hline P08195 & 4F2_HUMAN & 125 & 2000 & 0.939 & 4.977 & 0.839 & $125-2000$ & Depleted \\
\hline P08238 & HS90B_HUMAN & 125 & 2000 & 0.977 & 7.537 & 0.853 & $125-2000$ & Undepleted \\
\hline P08246 & ELNE_HUMAN & 500 & 2000 & 0.687 & 6.954 & 0.509 & $500-2000$ & Depleted \\
\hline P08294 & SODE_HUMAN & 2000 & 2000 & 0.821 & 4.421 & 0.507 & $N / A$ & Depleted \\
\hline P08473 & NEP_HUMAN & 250 & 2000 & 0.953 & 5.684 & 0.709 & $250-2000$ & Depleted \\
\hline P08519 & APOA_HUMAN & 500 & 2000 & 0.26 & 3.327 & 0.784 & $500-2000$ & Undepleted \\
\hline P08519 & APOA_HUMAN & 63 & 2000 & 0.863 & 0.686 & 1.569 & $63-2000$ & Depleted \\
\hline P08567 & PLEK_HUMAN & 63 & 2000 & 0.889 & 5.123 & 0.751 & $63-2000$ & Depleted \\
\hline P08571 & CD14_HUMAN & 1000 & 2000 & 0.663 & 5.008 & 0.43 & $1000-2000$ & Undepleted \\
\hline P08571 & CD14_HUMAN & 63 & 2000 & 0.965 & 4.863 & 1.027 & $63-2000$ & Depleted \\
\hline P08582 & TRFM_HUMAN & 250 & 2000 & 0.984 & 4.512 & 0.92 & $250-2000$ & Undepleted \\
\hline P08603 & CFAH_HUMAN & 63 & 2000 & 0.985 & 5.926 & 1.029 & $63-2000$ & Undepleted \\
\hline P08603 & CFAH_HUMAN & 63 & 2000 & 0.981 & 9.659 & 0.943 & $63-2000$ & Depleted \\
\hline P08637 & FCG3A_HUMAN & 250 & 2000 & 0.934 & 3.524 & 0.8 & $250-2000$ & Depleted \\
\hline P08670 & VIME_HUMAN & $\begin{array}{c}\text { not } \\
\text { quantifiable }\end{array}$ & & & & & & Depleted \\
\hline P08697 & A2AP_HUMAN & 63 & 2000 & 0.953 & 4.991 & 0.963 & $63-2000$ & Undepleted \\
\hline P08697 & A2AP_HUMAN & 63 & 2000 & 0.978 & 7.503 & 0.983 & $63-2000$ & Depleted \\
\hline P08727 & K1C19_HUMAN & 500 & 2000 & 0.918 & 5.311 & 0.813 & $500-2000$ & Depleted \\
\hline P08758 & ANXA5_HUMAN & 125 & 2000 & 0.977 & 6.196 & 0.806 & $125-2000$ & Undepleted \\
\hline P08865 & RSSA_HUMAN & 63 & 2000 & 0.934 & 5.705 & 0.936 & $63-2000$ & Undepleted \\
\hline P09172 & DOPO_HUMAN & 63 & 2000 & 0.926 & 0.48 & 1.447 & $63-2000$ & Depleted \\
\hline P09211 & GSTP1_HUMAN & 125 & 2000 & 0.978 & 6.573 & 0.807 & $125-2000$ & Undepleted \\
\hline P09211 & GSTP1_HUMAN & 250 & 2000 & 0.954 & 2.957 & 0.856 & $250-2000$ & Depleted \\
\hline P09622 & DLDH_HUMAN & $\begin{array}{c}\text { not } \\
\text { quantifiable }\end{array}$ & & & & & & Undepleted \\
\hline
\end{tabular}




\begin{tabular}{|c|c|c|c|c|c|c|c|c|}
\hline P09651 & ROA1_HUMAN & 500 & 2000 & 0.902 & 5.045 & 0.697 & $500-2000$ & Depleted \\
\hline P09871 & C1S_HUMAN & 63 & 2000 & 0.967 & 3.183 & 1.177 & $63-2000$ & Undepleted \\
\hline P09871 & C1S_HUMAN & 63 & 2000 & 0.968 & 6.789 & 1.021 & $63-2000$ & Depleted \\
\hline P09960 & LKHA4_HUMAN & 250 & 2000 & 0.982 & 5.846 & 0.855 & $250-2000$ & Undepleted \\
\hline P09972 & ALDOC_HUMAN & $\begin{array}{c}\text { not } \\
\text { quantifiable }\end{array}$ & & & & & & Undepleted \\
\hline P0C0L4 & CO4A_HUMAN & 125 & 2000 & 0.858 & 4.349 & 1.074 & $125-2000$ & Depleted \\
\hline P0C0L5 & CO4B_HUMAN & 63 & 2000 & 0.915 & 5.347 & 0.95 & $63-2000$ & Depleted \\
\hline P0DJI8 & SAA1_HUMAN & $\begin{array}{c}\text { not } \\
\text { quantifiable }\end{array}$ & & & & & & Undepleted \\
\hline P10155 & RO60_HUMAN & 125 & 2000 & 0.978 & 5.543 & 0.796 & $125-2000$ & Undepleted \\
\hline P10599 & THIO_HUMAN & 250 & 2000 & 0.981 & 5.664 & 0.682 & $250-2000$ & Undepleted \\
\hline P10643 & CO7_HUMAN & 63 & 2000 & 0.936 & 0.163 & 1.413 & $63-2000$ & Undepleted \\
\hline P10643 & CO7_HUMAN & 63 & 2000 & 0.982 & 7.069 & 0.991 & $63-2000$ & Depleted \\
\hline P10645 & CMGA_HUMAN & 125 & 2000 & 0.938 & 6.048 & 0.813 & $125-2000$ & Undepleted \\
\hline P10768 & ESTD_HUMAN & 250 & 2000 & 0.908 & 2.667 & 1.063 & $250-2000$ & Undepleted \\
\hline P10809 & CH60_HUMAN & 125 & 2000 & 0.648 & 6.265 & 1.003 & $125-2000$ & Undepleted \\
\hline P10809 & CH60_HUMAN & 63 & 2000 & 0.882 & 3.947 & 1.082 & $63-2000$ & Depleted \\
\hline P10909 & CLUS_HUMAN & 63 & 2000 & 0.985 & 5.961 & 1.028 & $63-2000$ & Undepleted \\
\hline P10909 & CLUS_HUMAN & 63 & 2000 & 0.984 & 8.488 & 0.877 & $63-2000$ & Depleted \\
\hline P11021 & GRP78_HUMAN & 63 & 2000 & 0.989 & 5.457 & 0.804 & $63-2000$ & Undepleted \\
\hline P11021 & GRP78_HUMAN & 1000 & 2000 & 0.823 & -1.522 & 1.373 & $1000-2000$ & Depleted \\
\hline P11047 & LAMC1_HUMAN & 125 & 2000 & 0.987 & 6.091 & 0.711 & $125-2000$ & Undepleted \\
\hline P11142 & HSP7C_HUMAN & 1000 & 2000 & 0.989 & -1.249 & 1.497 & $1000-2000$ & Depleted \\
\hline P11216 & PYGB_HUMAN & $\begin{array}{c}\text { not } \\
\text { quantifiable }\end{array}$ & & & & & & Undepleted \\
\hline P11226 & MBL2_HUMAN & 63 & 2000 & 0.959 & 1.383 & 1.255 & $63-2000$ & Depleted \\
\hline P11279 & LAMP1_HUMAN & 2000 & 2000 & 0.972 & 4.161 & 0.471 & $\mathrm{~N} / \mathrm{A}$ & Depleted \\
\hline P11413 & G6PD_HUMAN & 250 & 2000 & 0.876 & 5.772 & 0.689 & $250-2000$ & Depleted \\
\hline P11532 & DMD_HUMAN & 125 & 1000 & 0.837 & 3.126 & 0.912 & $125-1000$ & Depleted \\
\hline P11586 & C1TC_HUMAN & 125 & 2000 & 0.96 & 6.529 & 0.802 & $125-2000$ & Undepleted \\
\hline P11586 & C1TC_HUMAN & 250 & 2000 & 0.866 & 2.812 & 1.228 & $250-2000$ & Depleted \\
\hline P11717 & MPRI_HUMAN & 63 & 2000 & 0.902 & 3.144 & 0.988 & $63-2000$ & Depleted \\
\hline P11940 & PABP1_HUMAN & 125 & 2000 & 0.936 & 4.913 & 0.9 & $125-2000$ & Undepleted \\
\hline P12109 & CO6A1_HUMAN & 250 & 2000 & 0.976 & 4.315 & 0.891 & $250-2000$ & Undepleted \\
\hline P12109 & CO6A1_HUMAN & 1000 & 2000 & 0.596 & 4.768 & 0.379 & $1000-2000$ & Depleted \\
\hline P12110 & CO6A2_HUMAN & 125 & 2000 & 0.97 & 6.124 & 0.857 & $125-2000$ & Undepleted \\
\hline P12110 & CO6A2_HUMAN & 250 & 1000 & 0.921 & 5.151 & 0.779 & $250-1000$ & Depleted \\
\hline P12111 & CO6A3_HUMAN & 125 & 2000 & 0.972 & 6.456 & 0.814 & $125-2000$ & Undepleted \\
\hline $\mathrm{P} 12111$ & CO6A3_HUMAN & 63 & 2000 & 0.827 & 3.259 & 1.096 & $63-2000$ & Depleted \\
\hline P12259 & FA5_HUMAN & 1000 & 1000 & 0.066 & 5.735 & 0.345 & $\mathrm{~N} / \mathrm{A}$ & Undepleted \\
\hline P12259 & FA5_HUMAN & 63 & 2000 & 0.865 & 2.932 & 1.382 & $63-2000$ & Depleted \\
\hline $\mathrm{P} 12277$ & KCRB_HUMAN & 125 & 2000 & 0.963 & 6.966 & 0.763 & $125-2000$ & Undepleted \\
\hline P12277 & KCRB_HUMAN & 250 & 2000 & 0.852 & 3.447 & 0.908 & $250-2000$ & Depleted \\
\hline P12814 & ACTN1_HUMAN & 250 & 2000 & 0.861 & 4.436 & 0.871 & $250-2000$ & Undepleted \\
\hline
\end{tabular}




\begin{tabular}{|c|c|c|c|c|c|c|c|c|}
\hline P12814 & ACTN1_HUMAN & 125 & 2000 & 0.875 & 4.37 & 0.964 & $125-2000$ & Depleted \\
\hline P12931 & SRC_HUMAN & 63 & 2000 & 0.906 & 4.313 & 1.03 & $63-2000$ & Undepleted \\
\hline P12931 & SRC_HUMAN & 1000 & 2000 & 0.912 & 4.134 & 0.752 & $1000-2000$ & Depleted \\
\hline P12955 & PEPD_HUMAN & 125 & 2000 & 0.946 & 0.932 & 1.341 & $125-2000$ & Depleted \\
\hline P13473 & LAMP2_HUMAN & 125 & 2000 & 0.913 & 1.659 & 1.043 & $125-2000$ & Depleted \\
\hline P13521 & SCG2_HUMAN & 250 & 2000 & 0.934 & 4.99 & 0.766 & $250-2000$ & Depleted \\
\hline P13639 & EF2_HUMAN & 1000 & 2000 & 0.924 & 6.574 & 0.53 & $1000-2000$ & Depleted \\
\hline P13645 & K1C10_HUMAN & 500 & 2000 & 0.902 & 6.081 & 0.626 & $500-2000$ & Depleted \\
\hline P13667 & PDIA4_HUMAN & $\begin{array}{c}\text { not } \\
\text { quantifiable }\end{array}$ & & & & & & Undepleted \\
\hline P13671 & CO6_HUMAN & 63 & 2000 & 0.946 & 0.871 & 1.401 & $63-2000$ & Undepleted \\
\hline P13671 & CO6_HUMAN & 63 & 2000 & 0.985 & 7.402 & 0.934 & $63-2000$ & Depleted \\
\hline P13716 & HEM2_HUMAN & $\begin{array}{c}\text { not } \\
\text { quantifiable }\end{array}$ & & & & & & Depleted \\
\hline P13796 & PLSL_HUMAN & 63 & 2000 & 0.833 & 3.735 & 1.192 & $63-2000$ & Depleted \\
\hline P13797 & PLST_HUMAN & 250 & 2000 & 0.86 & 3.722 & 1.016 & $250-2000$ & Depleted \\
\hline P14151 & LYAM1_HUMAN & 63 & 2000 & 0.945 & 2.663 & 1.161 & $63-2000$ & Depleted \\
\hline P14174 & MIF_HUMAN & 250 & 2000 & 0.767 & 4.924 & 0.833 & $250-2000$ & Depleted \\
\hline P14314 & GLU2B_HUMAN & 125 & 2000 & 0.747 & 3.812 & 1.223 & $125-2000$ & Undepleted \\
\hline P14543 & NID1_HUMAN & 63 & 2000 & 0.845 & 3.962 & 0.823 & $63-2000$ & Depleted \\
\hline P14618 & KPYM_HUMAN & 63 & 2000 & 0.899 & 3.754 & 0.83 & $63-2000$ & Depleted \\
\hline P14625 & ENPL_HUMAN & 125 & 2000 & 0.757 & 6.747 & 0.872 & $125-2000$ & Undepleted \\
\hline P14927 & QCR7_HUMAN & $\begin{array}{c}\text { not } \\
\text { quantifiable }\end{array}$ & & & & & & Undepleted \\
\hline P15088 & CBPA3_HUMAN & 250 & 2000 & 0.785 & 4.592 & 0.785 & $250-2000$ & Depleted \\
\hline P15121 & ALDR_HUMAN & 63 & 2000 & 0.966 & 3.424 & 0.943 & $63-2000$ & Depleted \\
\hline P15144 & AMPN_HUMAN & 63 & 2000 & 0.924 & 3.366 & 1.144 & $63-2000$ & Depleted \\
\hline P15151 & PVR_HUMAN & 1000 & 2000 & 0.965 & 0.012 & 1.227 & $1000-2000$ & Depleted \\
\hline P15169 & CBPN_HUMAN & 500 & 2000 & 0.962 & 0.426 & 1.29 & $500-2000$ & Undepleted \\
\hline P15169 & CBPN_HUMAN & 63 & 2000 & 0.987 & 5.745 & 0.999 & $63-2000$ & Depleted \\
\hline P15311 & EZRI_HUMAN & 125 & 2000 & 0.771 & 5.114 & 0.775 & $125-2000$ & Depleted \\
\hline P15924 & DESP_HUMAN & 63 & 2000 & 0.871 & 3.918 & 0.946 & $63-2000$ & Depleted \\
\hline P16070 & CD44_HUMAN & 63 & 2000 & 0.946 & 3.237 & 1.126 & $63-2000$ & Depleted \\
\hline P16157 & ANK1_HUMAN & $\begin{array}{c}\text { not } \\
\text { quantifiable }\end{array}$ & & & & & & Undepleted \\
\hline P16233 & LIPP_HUMAN & $\begin{array}{c}\text { not } \\
\text { quantifiable }\end{array}$ & & & & & & Undepleted \\
\hline P16435 & NCPR_HUMAN & 125 & 1000 & 0.979 & 5.435 & 0.901 & $125-1000$ & Depleted \\
\hline P16949 & STMN1_HUMAN & 125 & 2000 & 0.981 & 2.267 & 1.118 & $125-2000$ & Depleted \\
\hline P17174 & AATC_HUMAN & 250 & 2000 & 0.889 & 4.511 & 0.818 & $250-2000$ & Depleted \\
\hline P17655 & CAN2_HUMAN & 250 & 2000 & 0.895 & 5.771 & 0.723 & $250-2000$ & Depleted \\
\hline P17661 & DESM_HUMAN & 250 & 2000 & 0.947 & 4.354 & 0.96 & $250-2000$ & Depleted \\
\hline P17844 & DDX5_HUMAN & 63 & 2000 & 0.951 & 3.368 & 0.945 & $63-2000$ & Undepleted \\
\hline P17900 & SAP3_HUMAN & 500 & 2000 & 0.81 & 3.009 & 1.169 & $500-2000$ & Depleted \\
\hline P17936 & IBP3_HUMAN & 500 & 2000 & 0.907 & 1.245 & 0.988 & $500-2000$ & Undepleted \\
\hline P17936 & IBP3_HUMAN & 63 & 2000 & 0.935 & 3.474 & 1.224 & $63-2000$ & Depleted \\
\hline P17980 & PRS6A_HUMAN & 63 & 2000 & 0.873 & 4.334 & 0.844 & $63-2000$ & Depleted \\
\hline
\end{tabular}




\begin{tabular}{|c|c|c|c|c|c|c|c|c|}
\hline P18065 & IBP2_HUMAN & 125 & 2000 & 0.963 & 5.936 & 0.789 & $125-2000$ & Undepleted \\
\hline P18065 & IBP2_HUMAN & 2000 & 2000 & 0.231 & 4.912 & 0.45 & $\mathrm{~N} / \mathrm{A}$ & Depleted \\
\hline P18124 & RL7_HUMAN & $\begin{array}{c}\text { not } \\
\text { quantifiable }\end{array}$ & & & & & & Depleted \\
\hline P18206 & VINC_HUMAN & 250 & 2000 & 0.886 & 6.393 & 0.582 & $250-2000$ & Depleted \\
\hline P18428 & LBP_HUMAN & 500 & 2000 & 0.943 & 1.121 & 1.122 & $500-2000$ & Undepleted \\
\hline P18428 & LBP_HUMAN & 63 & 2000 & 0.954 & 2.969 & 1.105 & $63-2000$ & Depleted \\
\hline P18669 & PGAM1_HUMAN & 125 & 2000 & 0.935 & 3.832 & 0.999 & $125-2000$ & Depleted \\
\hline P19012 & K1C15_HUMAN & 125 & 2000 & 0.932 & 6.101 & 0.707 & $125-2000$ & Depleted \\
\hline P19320 & VCAM1_HUMAN & 1000 & 2000 & 0.908 & 1.837 & 0.846 & $1000-2000$ & Depleted \\
\hline P19367 & HXK1_HUMAN & 125 & 2000 & 0.742 & 6.31 & 0.839 & $125-2000$ & Undepleted \\
\hline P19367 & HXK1_HUMAN & 63 & 1000 & 0.972 & 5.522 & 0.994 & $63-1000$ & Depleted \\
\hline P19652 & A1AG2_HUMAN & 63 & 2000 & 0.985 & 4.735 & 0.973 & $63-2000$ & Undepleted \\
\hline P19652 & A1AG2_HUMAN & 63 & 2000 & 0.981 & 4.578 & 0.905 & $63-2000$ & Depleted \\
\hline P19823 & ITIH2_HUMAN & 63 & 2000 & 0.979 & 6.261 & 1.006 & $63-2000$ & Undepleted \\
\hline P19823 & ITIH2_HUMAN & 63 & 2000 & 0.94 & 9.38 & 0.887 & $63-2000$ & Depleted \\
\hline P19827 & ITIH1_HUMAN & 63 & 2000 & 0.979 & 5.74 & 0.998 & $63-2000$ & Undepleted \\
\hline P19827 & ITIH1_HUMAN & 63 & 2000 & 0.945 & 8.155 & 1.037 & $63-2000$ & Depleted \\
\hline P19971 & TYPH_HUMAN & 500 & 2000 & 0.651 & 4.823 & 0.706 & $500-2000$ & Depleted \\
\hline P20290 & BTF3_HUMAN & 250 & 2000 & 0.898 & 3.242 & 0.945 & $250-2000$ & Depleted \\
\hline P20618 & PSB1_HUMAN & 1000 & 2000 & 0.872 & 3.974 & 0.812 & $1000-2000$ & Depleted \\
\hline P20674 & COX5A_HUMAN & 63 & 2000 & 0.902 & 3.667 & 1.05 & $63-2000$ & Depleted \\
\hline P20742 & PZP_HUMAN & 125 & 2000 & 0.803 & 4.815 & 0.852 & $125-2000$ & Undepleted \\
\hline P20742 & PZP_HUMAN & 125 & 2000 & 0.913 & 4.711 & 1.073 & $125-2000$ & Depleted \\
\hline P20774 & MIME_HUMAN & 1000 & 2000 & 0.64 & 4.335 & 0.476 & $1000-2000$ & Depleted \\
\hline P20810 & ICAL_HUMAN & 125 & 2000 & 0.899 & 4.019 & 0.916 & $125-2000$ & Depleted \\
\hline P20851 & C4BPB_HUMAN & 500 & 2000 & 0.895 & -1.597 & 1.587 & $500-2000$ & Undepleted \\
\hline P20851 & C4BPB_HUMAN & 63 & 2000 & 0.951 & 6.049 & 0.92 & $63-2000$ & Depleted \\
\hline P21291 & CSRP1_HUMAN & 125 & 2000 & 0.96 & 3.862 & 0.945 & $125-2000$ & Undepleted \\
\hline P21333 & FLNA_HUMAN & 250 & 2000 & 0.914 & 2.959 & 1.164 & $250-2000$ & Undepleted \\
\hline P21399 & ACOC_HUMAN & 250 & 2000 & 0.87 & 3.784 & 0.922 & $250-2000$ & Depleted \\
\hline P21695 & GPDA_HUMAN & 125 & 2000 & 0.801 & 3.996 & 0.942 & $125-2000$ & Depleted \\
\hline P21810 & PGS1_HUMAN & $\begin{array}{c}\text { not } \\
\text { quantifiable }\end{array}$ & & & & & & Undepleted \\
\hline P21912 & DHSB_HUMAN & 125 & 2000 & 0.954 & 5.593 & 0.928 & $125-2000$ & Undepleted \\
\hline P22061 & PIMT_HUMAN & 125 & 2000 & 0.924 & 5.872 & 0.797 & $125-2000$ & Undepleted \\
\hline P22061 & PIMT_HUMAN & 250 & 2000 & 0.978 & 3.135 & 0.858 & $250-2000$ & Depleted \\
\hline P22102 & PUR2_HUMAN & 125 & 2000 & 0.926 & 3.703 & 0.863 & $125-2000$ & Undepleted \\
\hline P22102 & PUR2_HUMAN & 250 & 2000 & 0.922 & 4.612 & 0.874 & $250-2000$ & Depleted \\
\hline P22105 & TENX_HUMAN & 500 & 2000 & 0.948 & 0.253 & 1.233 & $500-2000$ & Depleted \\
\hline P22234 & PUR6_HUMAN & 63 & 2000 & 0.941 & 4.066 & 0.914 & $63-2000$ & Depleted \\
\hline P22314 & UBA1_HUMAN & 63 & 2000 & 0.912 & 2.861 & 1.268 & $63-2000$ & Depleted \\
\hline P22352 & GPX3_HUMAN & 63 & 2000 & 0.968 & 2.329 & 0.939 & $63-2000$ & Undepleted \\
\hline P22352 & GPX3_HUMAN & 63 & 2000 & 0.97 & 5.058 & 0.978 & $63-2000$ & Depleted \\
\hline
\end{tabular}




\begin{tabular}{|c|c|c|c|c|c|c|c|c|}
\hline P22692 & IBP4_HUMAN & 1000 & 2000 & 0.911 & 2.437 & 0.89 & $1000-2000$ & Depleted \\
\hline P22792 & CPN2_HUMAN & 63 & 2000 & 0.955 & 2.144 & 1.245 & $63-2000$ & Undepleted \\
\hline P22792 & CPN2_HUMAN & 63 & 2000 & 0.948 & 6.925 & 0.948 & $63-2000$ & Depleted \\
\hline P22891 & PROZ_HUMAN & 125 & 2000 & 0.934 & 4.26 & 1.047 & $125-2000$ & Depleted \\
\hline P23083 & HV103_HUMAN & 63 & 2000 & 0.944 & 3.561 & 0.954 & $63-2000$ & Undepleted \\
\hline P23141 & EST1_HUMAN & $\begin{array}{c}\text { not } \\
\text { quantifiable }\end{array}$ & & & & & & Depleted \\
\hline P23142 & FBLN1_HUMAN & 125 & 2000 & 0.49 & 5.81 & 0.604 & $125-2000$ & Undepleted \\
\hline P23142 & FBLN1_HUMAN & 63 & 2000 & 0.949 & 1.6 & 1.249 & $63-2000$ & Depleted \\
\hline P23396 & RS3_HUMAN & 1000 & 1000 & 1 & 3.618 & 0.831 & $N / A$ & Depleted \\
\hline P23470 & PTPRG_HUMAN & 1000 & 2000 & 0.952 & 2.551 & 0.791 & $1000-2000$ & Depleted \\
\hline P23526 & SAHH_HUMAN & 63 & 2000 & 0.809 & 2.751 & 1.253 & $63-2000$ & Depleted \\
\hline P24298 & ALAT1_HUMAN & 500 & 2000 & 0.88 & 5.217 & 0.767 & $500-2000$ & Depleted \\
\hline P24592 & IBP6_HUMAN & 250 & 2000 & 0.888 & -1.283 & 1.484 & $250-2000$ & Depleted \\
\hline P24821 & TENA_HUMAN & 250 & 2000 & 0.644 & 5.399 & 0.723 & $250-2000$ & Undepleted \\
\hline P24821 & TENA_HUMAN & 125 & 2000 & 0.841 & 3.184 & 0.705 & $125-2000$ & Depleted \\
\hline P25311 & ZA2G_HUMAN & 63 & 2000 & 0.963 & 4.139 & 1.129 & $63-2000$ & Undepleted \\
\hline P25311 & ZA2G_HUMAN & 63 & 2000 & 0.988 & 7.566 & 0.911 & $63-2000$ & Depleted \\
\hline P25705 & ATPA_HUMAN & 125 & 2000 & 0.85 & 3.11 & 1.327 & $125-2000$ & Depleted \\
\hline P25774 & CATS_HUMAN & 500 & 2000 & 0.954 & 0.469 & 1.04 & $500-2000$ & Depleted \\
\hline P25786 & PSA1_HUMAN & $\begin{array}{c}\text { not } \\
\text { quantifiable }\end{array}$ & & & & & & Both \\
\hline P25787 & PSA2_HUMAN & $\begin{array}{c}\text { not } \\
\text { quantifiable }\end{array}$ & & & & & & Undepleted \\
\hline P25789 & PSA4_HUMAN & 250 & 2000 & 0.906 & 5.872 & 0.677 & $250-2000$ & Depleted \\
\hline P26196 & DDX6_HUMAN & 250 & 2000 & 0.929 & 2.835 & 0.856 & $250-2000$ & Depleted \\
\hline P26599 & PTBP1_HUMAN & 125 & 2000 & 0.971 & 6.316 & 0.788 & $125-2000$ & Undepleted \\
\hline P26639 & SYTC_HUMAN & $\begin{array}{c}\text { not } \\
\text { quantifiable }\end{array}$ & & & & & & Undepleted \\
\hline P26640 & SYVC_HUMAN & 63 & 2000 & 0.969 & 4.485 & 0.982 & $63-2000$ & Depleted \\
\hline P26927 & HGFL_HUMAN & 63 & 2000 & 0.962 & 3.19 & 1.082 & $63-2000$ & Depleted \\
\hline P27169 & PON1_HUMAN & 63 & 2000 & 0.914 & 3.665 & 1.173 & $63-2000$ & Undepleted \\
\hline P27169 & PON1_HUMAN & 63 & 2000 & 0.967 & 7.589 & 0.881 & $63-2000$ & Depleted \\
\hline P27348 & 1433T_HUMAN & $\begin{array}{c}\text { not } \\
\text { quantifiable }\end{array}$ & & & & & & Depleted \\
\hline P27487 & DPP4_HUMAN & 1000 & 2000 & 0.947 & -4.573 & 1.806 & $1000-2000$ & Depleted \\
\hline P27824 & CALX_HUMAN & 125 & 2000 & 0.903 & 5.77 & 0.984 & $125-2000$ & Undepleted \\
\hline P27824 & CALX_HUMAN & 250 & 2000 & 0.75 & 4.383 & 1.071 & $250-2000$ & Depleted \\
\hline P27918 & PROP_HUMAN & 500 & 2000 & 0.853 & -2.345 & 1.548 & $500-2000$ & Undepleted \\
\hline P27918 & PROP_HUMAN & 63 & 2000 & 0.992 & 5.354 & 1.066 & $63-2000$ & Depleted \\
\hline P28062 & PSB8_HUMAN & 500 & 2000 & 0.882 & 5.098 & 0.662 & $500-2000$ & Depleted \\
\hline P28066 & PSA5_HUMAN & 125 & 2000 & 0.872 & 6.049 & 0.608 & $125-2000$ & Undepleted \\
\hline P28070 & PSB4_HUMAN & 500 & 2000 & 0.83 & 7.764 & 0.455 & $500-2000$ & Depleted \\
\hline P28074 & PSB5_HUMAN & 125 & 2000 & 0.978 & 6.463 & 0.763 & $125-2000$ & Undepleted \\
\hline P28838 & AMPL_HUMAN & 250 & 2000 & 0.888 & 5.938 & 0.632 & $250-2000$ & Depleted \\
\hline P29144 & TPP2_HUMAN & 125 & 2000 & 0.601 & 5.63 & 1.073 & $125-2000$ & Undepleted \\
\hline
\end{tabular}




\begin{tabular}{|c|c|c|c|c|c|c|c|c|}
\hline P29350 & PTN6_HUMAN & $\begin{array}{c}\text { not } \\
\text { quantifiable }\end{array}$ & & & & & & Depleted \\
\hline P29401 & TKT_HUMAN & 250 & 2000 & 0.911 & 5.691 & 0.635 & $250-2000$ & Depleted \\
\hline P29622 & KAIN_HUMAN & 63 & 2000 & 0.971 & 3.13 & 0.949 & $63-2000$ & Undepleted \\
\hline P29622 & KAIN_HUMAN & 63 & 2000 & 0.985 & 7.017 & 0.991 & $63-2000$ & Depleted \\
\hline P30044 & PRDX5_HUMAN & 125 & 2000 & 0.877 & 4.245 & 0.956 & $125-2000$ & Depleted \\
\hline P30048 & PRDX3_HUMAN & 250 & 250 & 0.434 & 9.201 & $\begin{array}{c}- \\
0.129\end{array}$ & $\mathrm{~N} / \mathrm{A}$ & Depleted \\
\hline P30084 & ECHM_HUMAN & $\begin{array}{c}\text { not } \\
\text { quantifiable }\end{array}$ & & & & & & Undepleted \\
\hline P30085 & KCY_HUMAN & 250 & 2000 & 0.836 & 4.432 & 0.848 & $250-2000$ & Depleted \\
\hline P30101 & PDIA3_HUMAN & 63 & 2000 & 0.966 & 5.022 & 0.971 & $63-2000$ & Undepleted \\
\hline P30153 & 2AAA_HUMAN & 125 & 2000 & 0.908 & 3.785 & 1.005 & $125-2000$ & Depleted \\
\hline P30405 & PPIF_HUMAN & 250 & 2000 & 0.968 & 4.595 & 0.889 & $250-2000$ & Undepleted \\
\hline P30520 & PURA2_HUMAN & 125 & 2000 & 0.963 & 5.185 & 0.748 & $125-2000$ & Undepleted \\
\hline P31040 & DHSA_HUMAN & $\begin{array}{c}\text { not } \\
\text { quantifiable }\end{array}$ & & & & & & Undepleted \\
\hline P31146 & COR1A_HUMAN & 250 & 2000 & 0.93 & 5.749 & 0.593 & $250-2000$ & Depleted \\
\hline P31939 & PUR9_HUMAN & 63 & 1000 & 0.528 & 7.2 & 0.619 & $63-1000$ & Depleted \\
\hline P31943 & HNRH1_HUMAN & 125 & 2000 & 0.983 & 4.837 & 0.828 & $125-2000$ & Undepleted \\
\hline P31943 & HNRH1_HUMAN & 250 & 2000 & 0.811 & 5.974 & 0.705 & $250-2000$ & Depleted \\
\hline P31944 & CASPE_HUMAN & $\begin{array}{c}\text { not } \\
\text { quantifiable }\end{array}$ & & & & & & Undepleted \\
\hline P31946 & 1433B_HUMAN & 500 & 2000 & 0.79 & 3.705 & 0.853 & $500-2000$ & Undepleted \\
\hline P31946 & 1433B_HUMAN & 250 & 2000 & 0.923 & 6.11 & 0.721 & $250-2000$ & Depleted \\
\hline P31947 & 1433S_HUMAN & 125 & 2000 & 0.775 & 7.639 & 0.698 & $125-2000$ & Undepleted \\
\hline P31948 & STIP1_HUMAN & 1000 & 2000 & 0.914 & 2.238 & 1.085 & $1000-2000$ & Depleted \\
\hline P31949 & S10AB_HUMAN & 250 & 2000 & 0.843 & 5.547 & 0.678 & $250-2000$ & Depleted \\
\hline P31997 & CEAM8_HUMAN & $\begin{array}{c}\text { not } \\
\text { quantifiable }\end{array}$ & & & & & & Undepleted \\
\hline P32004 & L1CAM_HUMAN & 500 & 2000 & 0.974 & 5.939 & 0.81 & $500-2000$ & Undepleted \\
\hline P33151 & CADH5_HUMAN & 63 & 2000 & 0.959 & 0.709 & 1.434 & $63-2000$ & Depleted \\
\hline P33176 & KINH_HUMAN & 250 & 2000 & 0.811 & 3.041 & 1.255 & $250-2000$ & Depleted \\
\hline P33908 & MA1A1_HUMAN & 63 & 2000 & 0.967 & 0.037 & 1.337 & $63-2000$ & Depleted \\
\hline P33992 & MCM5_HUMAN & $\begin{array}{c}\text { not } \\
\text { quantifiable }\end{array}$ & & & & & & Undepleted \\
\hline P34896 & GLYC_HUMAN & $\begin{array}{c}\text { not } \\
\text { quantifiable }\end{array}$ & & & & & & Undepleted \\
\hline P34932 & HSP74_HUMAN & 63 & 2000 & 0.824 & 6.628 & 0.759 & $63-2000$ & Undepleted \\
\hline P34932 & HSP74_HUMAN & 63 & 2000 & 0.992 & 5.263 & 1.038 & $63-2000$ & Depleted \\
\hline P35052 & GPC1_HUMAN & 125 & 2000 & 0.687 & 6.07 & 0.894 & $125-2000$ & Undepleted \\
\hline P35232 & PHB_HUMAN & 250 & 2000 & 0.907 & 4.833 & 0.851 & $250-2000$ & Depleted \\
\hline P35237 & SPB6_HUMAN & 500 & 2000 & 0.858 & 4.665 & 0.669 & $500-2000$ & Depleted \\
\hline P35442 & TSP2_HUMAN & 250 & 2000 & 0.951 & 4.824 & 0.837 & $250-2000$ & Depleted \\
\hline P35520 & CBS_HUMAN & 125 & 2000 & 0.934 & 4.152 & 0.9 & $125-2000$ & Depleted \\
\hline P35527 & K1C9_HUMAN & $\begin{array}{c}\text { not } \\
\text { quantifiable }\end{array}$ & & & & & & Undepleted \\
\hline P35542 & SAA4_HUMAN & 63 & 2000 & 0.974 & 3.505 & 0.992 & $63-2000$ & Undepleted \\
\hline P35542 & SAA4_HUMAN & 2000 & 2000 & 0.919 & 4.839 & 0.395 & $\mathrm{~N} / \mathrm{A}$ & Depleted \\
\hline P35573 & GDE_HUMAN & 250 & 2000 & 0.688 & 6.818 & 0.549 & $250-2000$ & Depleted \\
\hline
\end{tabular}




\begin{tabular}{|c|c|c|c|c|c|c|c|c|}
\hline P35579 & MYH9_HUMAN & 63 & 2000 & 0.848 & 5.424 & 1.162 & $63-2000$ & Undepleted \\
\hline P35579 & MYH9_HUMAN & 63 & 2000 & 0.869 & 3.468 & 1.153 & $63-2000$ & Depleted \\
\hline P35754 & GLRX1_HUMAN & 250 & 2000 & 0.916 & 5.097 & 0.777 & $250-2000$ & Depleted \\
\hline P35858 & ALS_HUMAN & 63 & 2000 & 0.904 & 0.239 & 1.448 & $63-2000$ & Undepleted \\
\hline P35858 & ALS_HUMAN & 63 & 2000 & 0.972 & 7.613 & 0.873 & $63-2000$ & Depleted \\
\hline P35900 & K1C20_HUMAN & 125 & 2000 & 0.99 & 5.11 & 0.963 & $125-2000$ & Undepleted \\
\hline P35908 & K22E_HUMAN & 125 & 2000 & 0.83 & 4.287 & 1.103 & $125-2000$ & Undepleted \\
\hline P36222 & CH3L1_HUMAN & 500 & 2000 & 0.848 & 5.378 & 0.696 & $500-2000$ & Undepleted \\
\hline P36542 & ATPG_HUMAN & 63 & 2000 & 0.883 & 4.275 & 1.039 & $63-2000$ & Depleted \\
\hline P36578 & RL4_HUMAN & $\begin{array}{c}\text { not } \\
\text { quantifiable }\end{array}$ & & & & & & Undepleted \\
\hline P36871 & PGM1_HUMAN & 125 & 2000 & 0.915 & 4.24 & 0.908 & $125-2000$ & Depleted \\
\hline P36873 & PP1G_HUMAN & 500 & 2000 & 0.91 & 4.948 & 0.738 & $500-2000$ & Depleted \\
\hline P36955 & PEDF_HUMAN & 125 & 2000 & 0.956 & 2.592 & 1.16 & $125-2000$ & Undepleted \\
\hline P36955 & PEDF_HUMAN & 63 & 2000 & 0.981 & 7.273 & 0.94 & $63-2000$ & Depleted \\
\hline P36980 & FHR2_HUMAN & 1000 & 2000 & 0.92 & 2.595 & 0.798 & $1000-2000$ & Undepleted \\
\hline P36980 & FHR2_HUMAN & 125 & 2000 & 0.967 & 1.762 & 1.196 & $125-2000$ & Depleted \\
\hline P37802 & TAGL2_HUMAN & 250 & 2000 & 0.862 & 4.58 & 0.942 & $250-2000$ & Depleted \\
\hline P38606 & VATA_HUMAN & 125 & 2000 & 0.98 & 5.882 & 0.802 & $125-2000$ & Undepleted \\
\hline P38606 & VATA_HUMAN & 125 & 2000 & 0.862 & 3.793 & 0.837 & $125-2000$ & Depleted \\
\hline P38646 & GRP75_HUMAN & 63 & 2000 & 0.839 & 4.657 & 1.157 & $63-2000$ & Undepleted \\
\hline P39023 & RL3_HUMAN & 250 & 2000 & 0.768 & 4.372 & 0.897 & $250-2000$ & Undepleted \\
\hline P39059 & COFA1_HUMAN & 125 & 2000 & 0.949 & 4.311 & 0.892 & $125-2000$ & Undepleted \\
\hline P39060 & COIA1_HUMAN & 125 & 2000 & 0.616 & 6.067 & 0.982 & $125-2000$ & Undepleted \\
\hline P39060 & COIA1_HUMAN & 250 & 2000 & 0.665 & 4.837 & 0.804 & $250-2000$ & Depleted \\
\hline P40121 & CAPG_HUMAN & $\begin{array}{c}\text { not } \\
\text { quantifiable }\end{array}$ & & & & & & Depleted \\
\hline P40227 & TCPZ_HUMAN & 125 & 2000 & 0.958 & 3.788 & 0.951 & $125-2000$ & Undepleted \\
\hline P40763 & STAT3_HUMAN & 250 & 2000 & 0.879 & 5.629 & 0.756 & $250-2000$ & Depleted \\
\hline P40925 & MDHC_HUMAN & 63 & 2000 & 0.941 & 3.786 & 0.996 & $63-2000$ & Depleted \\
\hline P40939 & ECHA_HUMAN & $\begin{array}{c}\text { not } \\
\text { quantifiable }\end{array}$ & & & & & & Undepleted \\
\hline P41222 & PTGDS_HUMAN & $\begin{array}{c}\text { not } \\
\text { quantifiable }\end{array}$ & & & & & & Depleted \\
\hline P41250 & SYG_HUMAN & 125 & 2000 & 0.976 & 5.367 & 0.845 & $125-2000$ & Undepleted \\
\hline P41250 & SYG_HUMAN & 250 & 2000 & 0.847 & 4.1 & 1.011 & $250-2000$ & Depleted \\
\hline P42126 & ECl1_HUMAN & 1000 & 2000 & 0.971 & 4.811 & 0.743 & $1000-2000$ & Depleted \\
\hline P42166 & LAP2A_HUMAN & $\begin{array}{c}\text { not } \\
\text { quantifiable }\end{array}$ & & & & & & Undepleted \\
\hline P42574 & CASP3_HUMAN & 250 & 2000 & 0.895 & 4.145 & 0.936 & $250-2000$ & Undepleted \\
\hline P42765 & THIM_HUMAN & 125 & 2000 & 0.981 & 4.639 & 0.903 & $125-2000$ & Undepleted \\
\hline P42765 & THIM_HUMAN & 250 & 2000 & 0.926 & 5.368 & 0.735 & $250-2000$ & Depleted \\
\hline P43121 & MUC18_HUMAN & 250 & 2000 & 0.81 & 1.176 & 1.27 & $250-2000$ & Depleted \\
\hline P43243 & MATR3_HUMAN & 125 & 2000 & 0.896 & 3.145 & 1.005 & $125-2000$ & Depleted \\
\hline P43251 & BTD_HUMAN & 63 & 2000 & 0.982 & 5.179 & 0.975 & $63-2000$ & Depleted \\
\hline P43490 & NAMPT_HUMAN & 63 & 2000 & 0.901 & 4.233 & 0.939 & $63-2000$ & Depleted \\
\hline P43652 & AFAM_HUMAN & 63 & 2000 & 0.959 & 3.385 & 1.215 & $63-2000$ & Undepleted \\
\hline
\end{tabular}




\begin{tabular}{|c|c|c|c|c|c|c|c|c|}
\hline P43652 & AFAM_HUMAN & 63 & 2000 & 0.971 & 4.474 & 1.092 & $63-2000$ & Depleted \\
\hline P45974 & UBP5_HUMAN & 500 & 2000 & 0.801 & 4.599 & 0.726 & $500-2000$ & Depleted \\
\hline P46821 & MAP1B_HUMAN & 63 & 2000 & 0.823 & 6.366 & 0.857 & $63-2000$ & Depleted \\
\hline P46939 & UTRO_HUMAN & 125 & 2000 & 0.774 & 4.008 & 0.958 & $125-2000$ & Depleted \\
\hline P47756 & CAPZB_HUMAN & $\begin{array}{c}\text { not } \\
\text { quantifiable }\end{array}$ & & & & & & Depleted \\
\hline P47897 & SYQ_HUMAN & 125 & 2000 & 0.874 & 4.335 & 1.009 & $125-2000$ & Depleted \\
\hline P48047 & ATPO_HUMAN & 500 & 2000 & 0.705 & 5.178 & 0.641 & $500-2000$ & Depleted \\
\hline P48163 & MAOX_HUMAN & 125 & 2000 & 0.912 & 4.444 & 0.975 & $125-2000$ & Depleted \\
\hline P48506 & GSH1_HUMAN & 125 & 1000 & 0.985 & 7.534 & 0.776 & $125-1000$ & Undepleted \\
\hline P48681 & NEST_HUMAN & 125 & 2000 & 0.97 & 5.719 & 0.727 & $125-2000$ & Undepleted \\
\hline P48681 & NEST_HUMAN & 250 & 2000 & 0.908 & 6.221 & 0.573 & $250-2000$ & Depleted \\
\hline P48740 & MASP1_HUMAN & 63 & 2000 & 0.959 & 1.973 & 1.347 & $63-2000$ & Depleted \\
\hline P49005 & DPOD2_HUMAN & 125 & 2000 & 0.572 & 4.1 & 0.913 & $125-2000$ & Undepleted \\
\hline P49189 & AL9A1_HUMAN & 125 & 2000 & 0.873 & 6.673 & 0.836 & $125-2000$ & Undepleted \\
\hline P49189 & AL9A1_HUMAN & 500 & 2000 & 0.862 & 4.657 & 0.659 & $500-2000$ & Depleted \\
\hline P49321 & NASP_HUMAN & 125 & 2000 & 0.951 & 4.612 & 0.933 & $125-2000$ & Undepleted \\
\hline P49327 & FAS_HUMAN & 125 & 2000 & 0.828 & 6.259 & 0.843 & $125-2000$ & Undepleted \\
\hline P49327 & FAS_HUMAN & 125 & 2000 & 0.89 & 6.214 & 0.782 & $125-2000$ & Depleted \\
\hline P49588 & SYAC_HUMAN & 63 & 2000 & 0.933 & 1.819 & 1.324 & $63-2000$ & Depleted \\
\hline P49591 & SYSC_HUMAN & 63 & 1000 & 0.93 & 3.113 & 1.059 & $63-1000$ & Depleted \\
\hline P49593 & PPM1F_HUMAN & $\begin{array}{c}\text { not } \\
\text { quantifiable }\end{array}$ & & & & & & Depleted \\
\hline P49736 & MCM2_HUMAN & 250 & 2000 & 0.602 & 5.58 & 0.693 & $250-2000$ & Undepleted \\
\hline P49753 & ACOT2_HUMAN & 63 & 2000 & 0.881 & 3.864 & 0.892 & $63-2000$ & Depleted \\
\hline P49908 & SEPP1_HUMAN & 63 & 2000 & 0.96 & 1.412 & 1.218 & $63-2000$ & Undepleted \\
\hline P49908 & SEPP1_HUMAN & 63 & 2000 & 0.984 & 4.399 & 1.098 & $63-2000$ & Depleted \\
\hline P50213 & IDH3A_HUMAN & $\begin{array}{c}\text { not } \\
\text { quantifiable }\end{array}$ & & & & & & Undepleted \\
\hline P50281 & MMP14_HUMAN & 125 & 2000 & 0.963 & 6.089 & 0.766 & $125-2000$ & Undepleted \\
\hline P50395 & GDIB_HUMAN & 250 & 2000 & 0.903 & 4.175 & 0.89 & $250-2000$ & Undepleted \\
\hline P50395 & GDIB_HUMAN & 63 & 2000 & 0.893 & 4.176 & 0.834 & $63-2000$ & Depleted \\
\hline P50440 & GATM_HUMAN & 500 & 2000 & 0.903 & 4.659 & 0.753 & $500-2000$ & Depleted \\
\hline P50552 & VASP_HUMAN & 500 & 2000 & 0.865 & 5.317 & 0.632 & $500-2000$ & Depleted \\
\hline P50895 & BCAM_HUMAN & 500 & 2000 & 0.947 & 4.253 & 0.736 & $500-2000$ & Undepleted \\
\hline P50914 & RL14_HUMAN & 125 & 2000 & 0.923 & 6.144 & 0.915 & $125-2000$ & Undepleted \\
\hline P50990 & TCPQ_HUMAN & 250 & 2000 & 0.852 & 3.714 & 0.971 & $250-2000$ & Depleted \\
\hline P50991 & TCPD_HUMAN & 250 & 2000 & 0.928 & 5.19 & 0.688 & $250-2000$ & Depleted \\
\hline P51149 & RAB7A_HUMAN & 63 & 1000 & 0.969 & 5.588 & 0.828 & $63-1000$ & Depleted \\
\hline P51570 & GALK1_HUMAN & 125 & 2000 & 0.968 & 3.418 & 1.144 & $125-2000$ & Depleted \\
\hline P51610 & HCFC1_HUMAN & 63 & 2000 & 0.978 & 6.119 & 0.862 & $63-2000$ & Undepleted \\
\hline P51858 & HDGF_HUMAN & 63 & 2000 & 0.836 & 3.589 & 1.101 & $63-2000$ & Depleted \\
\hline P51884 & LUM_HUMAN & 63 & 2000 & 0.945 & 1.109 & 1.052 & $63-2000$ & Undepleted \\
\hline P51884 & LUM_HUMAN & 63 & 2000 & 0.984 & 6.301 & 0.957 & $63-2000$ & Depleted \\
\hline P51991 & ROA3_HUMAN & $\begin{array}{c}\text { not } \\
\text { quantifiable }\end{array}$ & & & & & & Undepleted \\
\hline
\end{tabular}




\begin{tabular}{|c|c|c|c|c|c|c|c|c|}
\hline P52209 & 6PGD_HUMAN & 125 & 2000 & 0.962 & 4.471 & 0.961 & $125-2000$ & Depleted \\
\hline P52272 & HNRPM_HUMAN & 125 & 2000 & 0.668 & 2.338 & 1.328 & $125-2000$ & Depleted \\
\hline P52565 & GDIR1_HUMAN & 250 & 2000 & 0.945 & 6.301 & 0.619 & $250-2000$ & Depleted \\
\hline P52701 & MSH6_HUMAN & 500 & 2000 & 0.833 & 4.879 & 0.762 & $500-2000$ & Depleted \\
\hline P52907 & CAZA1_HUMAN & 125 & 2000 & 0.99 & 7.113 & 0.8 & $125-2000$ & Undepleted \\
\hline P52907 & CAZA1_HUMAN & 500 & 2000 & 0.978 & 1.915 & 1.096 & $500-2000$ & Depleted \\
\hline P52948 & NUP98_HUMAN & 125 & 2000 & 0.837 & 4.01 & 0.988 & $125-2000$ & Depleted \\
\hline P53634 & CATC_HUMAN & 250 & 2000 & 0.927 & 4.234 & 0.864 & $250-2000$ & Undepleted \\
\hline P53634 & CATC_HUMAN & 125 & 2000 & 0.883 & 3.617 & 0.977 & $125-2000$ & Depleted \\
\hline P54108 & CRIS3_HUMAN & 1000 & 2000 & 0.928 & 0.42 & 1.089 & $1000-2000$ & Depleted \\
\hline P54136 & SYRC_HUMAN & 500 & 2000 & 0.98 & 4.609 & 0.748 & $500-2000$ & Depleted \\
\hline P54577 & SYYC_HUMAN & 125 & 2000 & 0.971 & 7.161 & 0.759 & $125-2000$ & Undepleted \\
\hline P54577 & SYYC_HUMAN & 125 & 2000 & 0.937 & 4.945 & 0.825 & $125-2000$ & Depleted \\
\hline P54578 & UBP14_HUMAN & 125 & 2000 & 0.808 & 4.559 & 1.123 & $125-2000$ & Undepleted \\
\hline P54727 & RD23B_HUMAN & $\begin{array}{c}\text { not } \\
\text { quantifiable }\end{array}$ & & & & & & Undepleted \\
\hline P54760 & EPHB4_HUMAN & 250 & 2000 & 0.848 & 5.432 & 0.944 & $250-2000$ & Undepleted \\
\hline P54802 & ANAG_HUMAN & 250 & 250 & 0.164 & 6.635 & 0.496 & $\mathrm{~N} / \mathrm{A}$ & Depleted \\
\hline P54886 & P5CS_HUMAN & $\begin{array}{c}\text { not } \\
\text { quantifiable }\end{array}$ & & & & & & Undepleted \\
\hline P55056 & APOC4_HUMAN & 250 & 2000 & 0.971 & 2.379 & 0.825 & $250-2000$ & Undepleted \\
\hline P55056 & APOC4_HUMAN & 2000 & 2000 & 0.941 & 1.946 & 0.791 & $\mathrm{~N} / \mathrm{A}$ & Depleted \\
\hline P55058 & PLTP_HUMAN & 2000 & 2000 & 0.978 & -0.476 & 1.176 & $\mathrm{~N} / \mathrm{A}$ & Undepleted \\
\hline P55058 & PLTP_HUMAN & 250 & 2000 & 0.986 & -0.816 & 1.228 & $250-2000$ & Depleted \\
\hline P55072 & TERA_HUMAN & 250 & 2000 & 0.806 & 2.469 & 1.169 & $250-2000$ & Depleted \\
\hline P55209 & NP1L1_HUMAN & 250 & 2000 & 0.813 & 5.545 & 0.93 & $250-2000$ & Undepleted \\
\hline P55285 & CADH6_HUMAN & 125 & 1000 & 0.839 & 1.545 & 1.16 & $125-1000$ & Depleted \\
\hline P55290 & CAD13_HUMAN & 1000 & 2000 & 0.953 & -1.01 & 1.283 & $1000-2000$ & Depleted \\
\hline P55786 & PSA_HUMAN & 63 & 2000 & 0.895 & 3.92 & 1.124 & $63-2000$ & Undepleted \\
\hline P55786 & PSA_HUMAN & 500 & 2000 & 0.833 & 7.015 & 0.477 & $500-2000$ & Depleted \\
\hline P55957 & BID_HUMAN & 125 & 2000 & 0.884 & 4.645 & 0.78 & $125-2000$ & Depleted \\
\hline P58107 & EPIPL_HUMAN & 125 & 2000 & 0.853 & 1.632 & 1.338 & $125-2000$ & Depleted \\
\hline P60660 & MYL6_HUMAN & 125 & 2000 & 0.934 & 3.07 & 1.083 & $125-2000$ & Depleted \\
\hline P60842 & IF4A1_HUMAN & 63 & 2000 & 0.944 & 4.008 & 0.916 & $63-2000$ & Depleted \\
\hline P60981 & DEST_HUMAN & 250 & 2000 & 0.738 & 4.767 & 0.689 & $250-2000$ & Depleted \\
\hline P61088 & UBE2N_HUMAN & 125 & 2000 & 0.924 & 4.481 & 0.802 & $125-2000$ & Depleted \\
\hline P61158 & ARP3_HUMAN & 250 & 2000 & 0.926 & 6.174 & 0.737 & $250-2000$ & Undepleted \\
\hline P61224 & RAP1B_HUMAN & $\begin{array}{c}\text { not } \\
\text { quantifiable }\end{array}$ & & & & & & Undepleted \\
\hline P61313 & RL15_HUMAN & 250 & 2000 & 0.617 & 5.754 & 0.49 & $250-2000$ & Depleted \\
\hline P61626 & LYSC_HUMAN & $\begin{array}{c}\text { not } \\
\text { quantifiable }\end{array}$ & & & & & & Undepleted \\
\hline P61764 & STXB1_HUMAN & 250 & 2000 & 0.96 & 6.214 & 0.723 & $250-2000$ & Undepleted \\
\hline P61769 & B2MG_HUMAN & 63 & 2000 & 0.989 & 2.308 & 1.096 & $63-2000$ & Depleted \\
\hline P61981 & 1433G_HUMAN & $\begin{array}{c}\text { not } \\
\text { quantifiable }\end{array}$ & & & & & & Depleted \\
\hline P62158 & CALM_HUMAN & 63 & 1000 & 0.965 & 4.044 & 0.899 & $63-1000$ & Depleted \\
\hline
\end{tabular}




\begin{tabular}{|c|c|c|c|c|c|c|c|c|}
\hline P62258 & 1433E_HUMAN & 125 & 2000 & 0.871 & 5.806 & 0.977 & $125-2000$ & Undepleted \\
\hline P62263 & RS14_HUMAN & 250 & 2000 & 0.753 & 5.163 & 0.913 & $250-2000$ & Depleted \\
\hline P62277 & RS13_HUMAN & $\begin{array}{c}\text { not } \\
\text { quantifiable }\end{array}$ & & & & & & Undepleted \\
\hline P62314 & SMD1_HUMAN & 500 & 2000 & 0.906 & 5.737 & 0.702 & $500-2000$ & Depleted \\
\hline P62701 & RS4X_HUMAN & 63 & 2000 & 0.917 & 4.823 & 0.976 & $63-2000$ & Depleted \\
\hline P62805 & H4_HUMAN & $\begin{array}{c}\text { not } \\
\text { quantifiable }\end{array}$ & & & & & & Depleted \\
\hline P62826 & RAN_HUMAN & 250 & 2000 & 0.925 & 5.638 & 0.668 & $250-2000$ & Depleted \\
\hline P62857 & RS28_HUMAN & 125 & 2000 & 0.966 & 6.705 & 0.761 & $125-2000$ & Undepleted \\
\hline P62906 & RL10A_HUMAN & $\begin{array}{c}\text { not } \\
\text { quantifiable }\end{array}$ & & & & & & Undepleted \\
\hline P62942 & FKB1A_HUMAN & 125 & 1000 & 0.945 & 4.208 & 0.859 & $125-1000$ & Undepleted \\
\hline P62993 & GRB2_HUMAN & 63 & 2000 & 0.83 & 6.015 & 0.683 & $63-2000$ & Depleted \\
\hline P63104 & 1433Z_HUMAN & 125 & 2000 & 0.926 & 5.013 & 0.781 & $125-2000$ & Undepleted \\
\hline P63104 & 1433Z_HUMAN & 500 & 2000 & 0.919 & 4.011 & 0.899 & $500-2000$ & Depleted \\
\hline P63313 & TYB10_HUMAN & 125 & 2000 & 0.973 & 1.874 & 1.202 & $125-2000$ & Depleted \\
\hline P67809 & YBOX1_HUMAN & 63 & 2000 & 0.981 & 4.157 & 1.04 & $63-2000$ & Undepleted \\
\hline P68366 & TBA4A_HUMAN & 500 & 2000 & 0.779 & 6.546 & 0.605 & $500-2000$ & Depleted \\
\hline P68871 & HBB_HUMAN & 63 & 2000 & 0.904 & 1.426 & 1.638 & $63-2000$ & Undepleted \\
\hline P68871 & HBB_HUMAN & 250 & 2000 & 0.923 & 5.503 & 0.824 & $250-2000$ & Depleted \\
\hline P69905 & HBA_HUMAN & 63 & 2000 & 0.935 & 4.296 & 0.915 & $63-2000$ & Undepleted \\
\hline P78347 & GTF2I_HUMAN & 250 & 2000 & 0.788 & 5.318 & 0.923 & $250-2000$ & Depleted \\
\hline P78417 & GSTO1_HUMAN & $\begin{array}{c}\text { not } \\
\text { quantifiable }\end{array}$ & & & & & & Depleted \\
\hline P78527 & PRKDC_HUMAN & 250 & 2000 & 0.919 & 4.825 & 0.829 & $250-2000$ & Undepleted \\
\hline P78527 & PRKDC_HUMAN & 63 & 2000 & 0.983 & 6.241 & 1.068 & $63-2000$ & Depleted \\
\hline P80108 & PHLD_HUMAN & 125 & 2000 & 0.441 & 6.414 & 0.466 & $125-2000$ & Undepleted \\
\hline P80108 & PHLD_HUMAN & 63 & 2000 & 0.977 & 4.871 & 1.067 & $63-2000$ & Depleted \\
\hline P84243 & H33_HUMAN & 250 & 2000 & 0.836 & 1.21 & 1.396 & $250-2000$ & Depleted \\
\hline P98082 & DAB2_HUMAN & $\begin{array}{c}\text { not } \\
\text { quantifiable }\end{array}$ & & & & & & Depleted \\
\hline P98160 & PGBM_HUMAN & 500 & 2000 & 0.981 & -4.712 & 1.905 & $500-2000$ & Depleted \\
\hline Q00610 & CLH1_HUMAN & 63 & 2000 & 0.872 & 4.632 & 1.145 & $63-2000$ & Depleted \\
\hline Q00839 & HNRPU_HUMAN & 125 & 2000 & 0.838 & 5.305 & 0.909 & $125-2000$ & Undepleted \\
\hline Q00839 & HNRPU_HUMAN & 125 & 2000 & 0.658 & 3.927 & 0.858 & $125-2000$ & Depleted \\
\hline Q01082 & SPTB2_HUMAN & 250 & 2000 & 0.819 & 4.253 & 0.901 & $250-2000$ & Depleted \\
\hline Q01459 & DIAC_HUMAN & 500 & 2000 & 0.792 & 3.682 & 0.698 & $500-2000$ & Depleted \\
\hline Q01581 & HMCS1_HUMAN & $\begin{array}{c}\text { not } \\
\text { quantifiable }\end{array}$ & & & & & & Depleted \\
\hline Q02790 & FKBP4_HUMAN & 63 & 1000 & 0.956 & 5.029 & 0.799 & $63-1000$ & Undepleted \\
\hline Q02790 & FKBP4_HUMAN & 63 & 2000 & 0.815 & 7.291 & 0.799 & $63-2000$ & Depleted \\
\hline Q02809 & PLOD1_HUMAN & 500 & 2000 & 0.872 & 5.133 & 0.675 & $500-2000$ & Depleted \\
\hline Q02880 & TOP2B_HUMAN & 125 & 2000 & 0.898 & 5.313 & 0.817 & $125-2000$ & Undepleted \\
\hline Q02880 & TOP2B_HUMAN & 125 & 2000 & 0.987 & 4.278 & 0.932 & $125-2000$ & Depleted \\
\hline Q02952 & AKA12_HUMAN & 125 & 2000 & 0.973 & 5.58 & 0.839 & $125-2000$ & Undepleted \\
\hline Q03154 & ACY1_HUMAN & 125 & 2000 & 0.973 & 4.697 & 0.862 & $125-2000$ & Undepleted \\
\hline
\end{tabular}




\begin{tabular}{|c|c|c|c|c|c|c|c|c|}
\hline Q03591 & FHR1_HUMAN & 1000 & 1000 & 0.949 & 3.454 & 0.799 & $\mathrm{~N} / \mathrm{A}$ & Undepleted \\
\hline Q03591 & FHR1_HUMAN & 1000 & 2000 & 0.708 & -0.616 & 1.296 & $1000-2000$ & Depleted \\
\hline Q04756 & HGFA_HUMAN & 125 & 2000 & 0.917 & 1.939 & 1.326 & $125-2000$ & Depleted \\
\hline Q04760 & LGUL_HUMAN & $\begin{array}{c}\text { not } \\
\text { quantifiable }\end{array}$ & & & & & & Depleted \\
\hline Q04917 & 1433F_HUMAN & 63 & 1000 & 0.934 & 5.091 & 1.072 & $63-1000$ & Undepleted \\
\hline Q04917 & 1433F_HUMAN & 125 & 2000 & 0.884 & 4.267 & 0.836 & $125-2000$ & Depleted \\
\hline Q05397 & FAK1_HUMAN & 250 & 2000 & 0.895 & 5.448 & 0.697 & $250-2000$ & Depleted \\
\hline Q05707 & COEA1_HUMAN & 125 & 2000 & 0.964 & 7.11 & 0.693 & $125-2000$ & Undepleted \\
\hline Q05707 & COEA1_HUMAN & 125 & 2000 & 0.805 & 2.647 & 1.179 & $125-2000$ & Depleted \\
\hline Q06033 & ITIH3_HUMAN & 125 & 2000 & 0.901 & 0.481 & 1.32 & $125-2000$ & Undepleted \\
\hline Q06033 & ITIH3_HUMAN & 63 & 2000 & 0.985 & 5.957 & 1.006 & $63-2000$ & Depleted \\
\hline Q06830 & PRDX1_HUMAN & $\begin{array}{c}\text { not } \\
\text { quantifiable }\end{array}$ & & & & & & Undepleted \\
\hline Q07912 & ACK1_HUMAN & $\begin{array}{c}\text { not } \\
\text { quantifiable }\end{array}$ & & & & & & Undepleted \\
\hline Q07960 & RHG01_HUMAN & 125 & 2000 & 0.896 & 4.936 & 0.702 & $125-2000$ & Depleted \\
\hline Q08209 & PP2BA_HUMAN & 500 & 2000 & 0.921 & 6.324 & 0.581 & $500-2000$ & Depleted \\
\hline Q08257 & QOR_HUMAN & 500 & 2000 & 0.88 & 4.652 & 0.717 & $500-2000$ & Depleted \\
\hline Q08380 & LG3BP_HUMAN & 500 & 2000 & 0.775 & -0.02 & 1.209 & $500-2000$ & Undepleted \\
\hline Q08380 & LG3BP_HUMAN & 63 & 2000 & 0.97 & 3.883 & 1.169 & $63-2000$ & Depleted \\
\hline Q09666 & AHNK_HUMAN & 125 & 2000 & 0.822 & 7.757 & 0.827 & $125-2000$ & Depleted \\
\hline Q10567 & AP1B1_HUMAN & 250 & 2000 & 0.76 & 3.167 & 0.941 & $250-2000$ & Depleted \\
\hline Q10588 & BST1_HUMAN & 500 & 2000 & 0.911 & 0.591 & 1.171 & $500-2000$ & Depleted \\
\hline Q12805 & FBLN3_HUMAN & 125 & 2000 & 0.937 & 0.885 & 1.275 & $125-2000$ & Depleted \\
\hline Q12841 & FSTL1_HUMAN & 2000 & 2000 & 0.508 & 4.511 & 0.362 & $\mathrm{~N} / \mathrm{A}$ & Depleted \\
\hline Q12906 & ILF3_HUMAN & 125 & 2000 & 0.975 & 3.716 & 0.859 & $125-2000$ & Undepleted \\
\hline Q12906 & ILF3_HUMAN & 63 & 2000 & 0.954 & 5.768 & 0.803 & $63-2000$ & Depleted \\
\hline Q12931 & TRAP1_HUMAN & 63 & 2000 & 0.969 & 2.79 & 1.042 & $63-2000$ & Depleted \\
\hline Q13045 & FLII_HUMAN & 250 & 2000 & 0.898 & 4.716 & 0.753 & $250-2000$ & Depleted \\
\hline Q13103 & SPP24_HUMAN & $\begin{array}{c}\text { not } \\
\text { quantifiable }\end{array}$ & & & & & & Depleted \\
\hline Q13185 & CBX3_HUMAN & 125 & 2000 & 0.904 & 4.975 & 0.806 & $125-2000$ & Depleted \\
\hline Q13200 & PSMD2_HUMAN & 63 & 2000 & 0.827 & 3.937 & 1.027 & $63-2000$ & Depleted \\
\hline Q13232 & NDK3_HUMAN & 63 & 2000 & 0.969 & 5.767 & 0.855 & $63-2000$ & Undepleted \\
\hline Q13442 & HAP28_HUMAN & 63 & 2000 & 0.974 & 4.922 & 0.984 & $63-2000$ & Depleted \\
\hline Q13451 & FKBP5_HUMAN & 63 & 250 & 0.708 & 8.748 & 0.648 & $63-250$ & Undepleted \\
\hline Q13509 & TBB3_HUMAN & $\begin{array}{c}\text { not } \\
\text { quantifiable }\end{array}$ & & & & & & Undepleted \\
\hline Q13557 & KCC2D_HUMAN & 250 & 2000 & 0.888 & 3.604 & 0.981 & $250-2000$ & Depleted \\
\hline Q13564 & ULA1_HUMAN & 500 & 2000 & 0.922 & 5.087 & 0.688 & $500-2000$ & Depleted \\
\hline Q13576 & IQGA2_HUMAN & 250 & 2000 & 0.958 & 5.639 & 0.722 & $250-2000$ & Undepleted \\
\hline Q13576 & IQGA2_HUMAN & 125 & 2000 & 0.995 & 3.497 & 0.952 & $125-2000$ & Depleted \\
\hline Q13630 & FCL_HUMAN & $\begin{array}{c}\text { not } \\
\text { quantifiable }\end{array}$ & & & & & & Undepleted \\
\hline Q13740 & CD166_HUMAN & 63 & 2000 & 0.894 & 4.749 & 0.904 & $63-2000$ & Depleted \\
\hline Q13765 & NACA_HUMAN & 250 & 2000 & 0.852 & 6.359 & 0.493 & $250-2000$ & Depleted \\
\hline
\end{tabular}




\begin{tabular}{|c|c|c|c|c|c|c|c|c|}
\hline Q13790 & APOF_HUMAN & 125 & 2000 & 0.922 & 0.627 & 1.3 & $125-2000$ & Depleted \\
\hline Q14012 & KCC1A_HUMAN & 125 & 2000 & 0.948 & 5.425 & 0.78 & $125-2000$ & Undepleted \\
\hline Q14103 & HNRPD_HUMAN & 125 & 2000 & 0.981 & 6.836 & 0.809 & $125-2000$ & Undepleted \\
\hline Q14103 & HNRPD_HUMAN & 250 & 1000 & 0.91 & 3.711 & 0.97 & $250-1000$ & Depleted \\
\hline Q14126 & DSG2_HUMAN & $\begin{array}{c}\text { not } \\
\text { quantifiable }\end{array}$ & & & & & & Depleted \\
\hline Q14203 & DCTN1_HUMAN & 2000 & 2000 & 0.988 & 0.327 & 1.147 & $\mathrm{~N} / \mathrm{A}$ & Depleted \\
\hline Q14204 & DYHC1_HUMAN & 250 & 2000 & 0.764 & 3.043 & 1.314 & $250-2000$ & Undepleted \\
\hline Q14204 & DYHC1_HUMAN & 63 & 2000 & 0.885 & 3.381 & 1.258 & $63-2000$ & Depleted \\
\hline Q14247 & SRC8_HUMAN & 125 & 2000 & 0.937 & 6.05 & 0.779 & $125-2000$ & Undepleted \\
\hline Q14258 & TRI25_HUMAN & 125 & 2000 & 0.805 & 5.021 & 0.871 & $125-2000$ & Depleted \\
\hline Q14289 & FAK2_HUMAN & 125 & 2000 & 0.957 & 5.239 & 0.896 & $125-2000$ & Undepleted \\
\hline Q14289 & FAK2_HUMAN & 63 & 2000 & 0.941 & 3.97 & 1.224 & $63-2000$ & Depleted \\
\hline Q14315 & FLNC_HUMAN & 125 & 2000 & 0.78 & 5.306 & 1.033 & $125-2000$ & Undepleted \\
\hline Q14315 & FLNC_HUMAN & 125 & 2000 & 0.82 & 4.316 & 0.921 & $125-2000$ & Depleted \\
\hline Q14520 & HABP2_HUMAN & 250 & 2000 & 0.924 & 1.674 & 1.188 & $250-2000$ & Undepleted \\
\hline Q14520 & HABP2_HUMAN & 63 & 2000 & 0.992 & 5.495 & 1.014 & $63-2000$ & Depleted \\
\hline Q14624 & ITIH4_HUMAN & 63 & 2000 & 0.983 & 5.816 & 1.056 & $63-2000$ & Undepleted \\
\hline Q14624 & ITIH4_HUMAN & 63 & 2000 & 0.976 & 8.471 & 0.964 & $63-2000$ & Depleted \\
\hline Q14690 & RRP5_HUMAN & 250 & 2000 & 0.972 & 4.373 & 0.816 & $250-2000$ & Undepleted \\
\hline Q14690 & RRP5_HUMAN & 125 & 2000 & 0.998 & 4.906 & 0.928 & $125-2000$ & Depleted \\
\hline Q14789 & GOGB1_HUMAN & 250 & 2000 & 0.956 & 3.432 & 0.842 & $250-2000$ & Undepleted \\
\hline Q14789 & GOGB1_HUMAN & 250 & 2000 & 0.915 & 4.727 & 0.725 & $250-2000$ & Depleted \\
\hline Q14974 & IMB1_HUMAN & 63 & 2000 & 0.907 & 2.197 & 1.285 & $63-2000$ & Depleted \\
\hline Q15029 & U5S1_HUMAN & 125 & 2000 & 0.963 & 5.617 & 0.818 & $125-2000$ & Undepleted \\
\hline Q15029 & U5S1_HUMAN & 125 & 2000 & 0.947 & 4.788 & 0.819 & $125-2000$ & Depleted \\
\hline Q15056 & IF4H_HUMAN & 63 & 2000 & 0.917 & 3.064 & 1.08 & $63-2000$ & Depleted \\
\hline Q15063 & POSTN_HUMAN & 63 & 2000 & 0.993 & 5.403 & 0.928 & $63-2000$ & Depleted \\
\hline Q15084 & PDIA6_HUMAN & $\begin{array}{c}\text { not } \\
\text { quantifiable }\end{array}$ & & & & & & Undepleted \\
\hline Q15113 & PCOC1_HUMAN & 250 & 2000 & 0.938 & 2.545 & 1.075 & $250-2000$ & Depleted \\
\hline Q15149 & PLEC_HUMAN & 250 & 2000 & 0.957 & 4.345 & 0.841 & $250-2000$ & Undepleted \\
\hline Q15149 & PLEC_HUMAN & 63 & 2000 & 0.913 & 3.43 & 1.182 & $63-2000$ & Depleted \\
\hline Q15166 & PON3_HUMAN & 500 & 2000 & 0.989 & 3.857 & 0.898 & $500-2000$ & Undepleted \\
\hline Q15166 & PON3_HUMAN & 63 & 2000 & 0.871 & 6.362 & 0.843 & $63-2000$ & Depleted \\
\hline Q15185 & TEBP_HUMAN & 125 & 2000 & 0.973 & 4.7 & 0.867 & $125-2000$ & Undepleted \\
\hline Q15366 & PCBP2_HUMAN & 125 & 2000 & 0.906 & 3.661 & 0.925 & $125-2000$ & Undepleted \\
\hline Q15369 & ELOC_HUMAN & $\begin{array}{c}\text { not } \\
\text { quantifiable }\end{array}$ & & & & & & Undepleted \\
\hline Q15404 & RSU1_HUMAN & 500 & 2000 & 0.83 & 6.685 & 0.505 & $500-2000$ & Depleted \\
\hline Q15428 & SF3A2_HUMAN & 125 & 2000 & 0.831 & 7.189 & 0.775 & $125-2000$ & Undepleted \\
\hline Q15582 & BGH3_HUMAN & 2000 & 2000 & 0.759 & 1.428 & 0.9 & $\mathrm{~N} / \mathrm{A}$ & Undepleted \\
\hline Q15582 & BGH3_HUMAN & 250 & 2000 & 0.848 & 0.931 & 1.481 & $250-2000$ & Depleted \\
\hline Q15631 & TSN_HUMAN & 500 & 2000 & 0.907 & 4.131 & 0.875 & $500-2000$ & Depleted \\
\hline Q15717 & ELAV1_HUMAN & 125 & 2000 & 0.851 & 3.038 & 1.215 & $125-2000$ & Depleted \\
\hline
\end{tabular}




\begin{tabular}{|c|c|c|c|c|c|c|c|c|}
\hline Q15746 & MYLK_HUMAN & 250 & 2000 & 0.83 & 4.881 & 0.956 & $250-2000$ & Depleted \\
\hline Q15833 & STXB2_HUMAN & 250 & 2000 & 0.953 & 5.579 & 0.745 & $250-2000$ & Undepleted \\
\hline Q15833 & STXB2_HUMAN & 63 & 2000 & 0.892 & 3.49 & 0.899 & $63-2000$ & Depleted \\
\hline Q15848 & ADIPO_HUMAN & 2000 & 2000 & 0.869 & 2.36 & 0.741 & $\mathrm{~N} / \mathrm{A}$ & Undepleted \\
\hline Q15848 & ADIPO_HUMAN & 250 & 2000 & 0.769 & 0.287 & 1.214 & $250-2000$ & Depleted \\
\hline Q15942 & ZYX_HUMAN & 250 & 2000 & 0.959 & 4.798 & 0.783 & $250-2000$ & Depleted \\
\hline Q16363 & LAMA4_HUMAN & 1000 & 2000 & 0.91 & 2.489 & 1.001 & $1000-2000$ & Depleted \\
\hline Q16401 & PSMD5_HUMAN & 63 & 2000 & 0.905 & 3.571 & 1.111 & $63-2000$ & Depleted \\
\hline Q16531 & DDB1_HUMAN & 250 & 2000 & 0.872 & 4.915 & 0.755 & $250-2000$ & Depleted \\
\hline Q16555 & DPYL2_HUMAN & 125 & 2000 & 0.951 & 3.639 & 0.918 & $125-2000$ & Depleted \\
\hline Q16586 & SGCA_HUMAN & 125 & 2000 & 0.935 & 1.549 & 1.039 & $125-2000$ & Depleted \\
\hline Q16610 & ECM1_HUMAN & 500 & 2000 & 0.884 & -3.488 & 1.829 & $500-2000$ & Undepleted \\
\hline Q16610 & ECM1_HUMAN & 63 & 2000 & 0.986 & 6.508 & 0.966 & $63-2000$ & Depleted \\
\hline Q16658 & FSCN1_HUMAN & 500 & 2000 & 0.916 & 5.309 & 0.698 & $500-2000$ & Depleted \\
\hline Q16719 & KYNU_HUMAN & $\begin{array}{c}\text { not } \\
\text { quantifiable }\end{array}$ & & & & & & Undepleted \\
\hline Q16775 & GLO2_HUMAN & 250 & 2000 & 0.965 & 4.961 & 0.789 & $250-2000$ & Undepleted \\
\hline Q16822 & PCKGM_HUMAN & 63 & 2000 & 0.989 & 3.379 & 0.93 & $63-2000$ & Depleted \\
\hline Q16851 & UGPA_HUMAN & 125 & 2000 & 0.919 & 6.088 & 0.946 & $125-2000$ & Undepleted \\
\hline Q16851 & UGPA_HUMAN & 250 & 2000 & 0.909 & 4.075 & 0.726 & $250-2000$ & Depleted \\
\hline Q16853 & AOC3_HUMAN & 1000 & 2000 & 0.913 & 1.882 & 0.913 & $1000-2000$ & Depleted \\
\hline Q1KMD3 & HNRL2_HUMAN & 250 & 2000 & 0.714 & 4.03 & 1.068 & $250-2000$ & Undepleted \\
\hline Q27J81 & INF2_HUMAN & 63 & 2000 & 0.929 & 5.014 & 0.804 & $63-2000$ & Depleted \\
\hline Q2PZI1 & D19L1_HUMAN & 63 & 250 & 0.951 & 7.87 & 0.654 & $63-250$ & Undepleted \\
\hline Q3LXA3 & DHAK_HUMAN & $\begin{array}{c}\text { not } \\
\text { quantifiable }\end{array}$ & & & & & & Undepleted \\
\hline Q4VC31 & CCD58_HUMAN & 63 & 2000 & 0.985 & 5.023 & 0.922 & $63-2000$ & Depleted \\
\hline Q53FA7 & QORX_HUMAN & $\begin{array}{c}\text { not } \\
\text { quantifiable }\end{array}$ & & & & & & Depleted \\
\hline Q53GG5 & PDLI3_HUMAN & 1000 & 2000 & 0.845 & 1.645 & 1.246 & $1000-2000$ & Depleted \\
\hline Q5JRA6 & MIA3_HUMAN & $\begin{array}{c}\text { not } \\
\text { quantifiable }\end{array}$ & & & & & & Undepleted \\
\hline Q5JSH3 & WDR44_HUMAN & 63 & 2000 & 0.924 & 4.15 & 0.928 & $63-2000$ & Depleted \\
\hline Q5SYB0 & FRPD1_HUMAN & 2000 & 2000 & 0.954 & 2.64 & 0.775 & $\mathrm{~N} / \mathrm{A}$ & Undepleted \\
\hline Q5SYB0 & FRPD1_HUMAN & 250 & 2000 & 0.945 & 2.051 & 0.997 & $250-2000$ & Depleted \\
\hline Q5T013 & HYI_HUMAN & 63 & 2000 & 0.993 & 4.232 & 1.003 & $63-2000$ & Depleted \\
\hline Q5TFE4 & NT5D1_HUMAN & 63 & 2000 & 0.845 & 4.099 & 0.995 & $63-2000$ & Depleted \\
\hline Q5VUA4 & ZN318_HUMAN & 63 & 2000 & 0.906 & 4.228 & 0.885 & $63-2000$ & Undepleted \\
\hline Q5VUA4 & ZN318_HUMAN & 2000 & 2000 & 0.067 & 9.44 & 0.167 & $\mathrm{~N} / \mathrm{A}$ & Depleted \\
\hline Q6EMK4 & VASN_HUMAN & 63 & 2000 & 0.972 & 1.027 & 1.42 & $63-2000$ & Depleted \\
\hline Q6NZI2 & PTRF_HUMAN & $\begin{array}{c}\text { not } \\
\text { quantifiable }\end{array}$ & & & & & & Undepleted \\
\hline Q6P179 & ERAP2_HUMAN & 125 & 2000 & 0.967 & 7.367 & 0.758 & $125-2000$ & Undepleted \\
\hline Q6PI48 & SYDM_HUMAN & 125 & 2000 & 0.639 & 8.17 & 0.612 & $125-2000$ & Undepleted \\
\hline Q6PKG0 & LARP1_HUMAN & 63 & 2000 & 0.854 & 2.686 & 1.21 & $63-2000$ & Depleted \\
\hline Q6UWP8 & SBSN_HUMAN & 125 & 2000 & 0.984 & 4.167 & 0.812 & $125-2000$ & Undepleted \\
\hline
\end{tabular}




\begin{tabular}{|c|c|c|c|c|c|c|c|c|}
\hline Q6UX71 & PXDC2_HUMAN & $\begin{array}{c}\text { not } \\
\text { quantifiable }\end{array}$ & & & & & & Depleted \\
\hline Q6UXB8 & PI16_HUMAN & 250 & 2000 & 0.943 & 0.4 & 1.342 & $250-2000$ & Depleted \\
\hline Q6XQN6 & PNCB_HUMAN & 250 & 2000 & 0.959 & 6.627 & 0.719 & $250-2000$ & Undepleted \\
\hline Q6XQN6 & PNCB_HUMAN & 250 & 2000 & 0.738 & 5.152 & 0.767 & $250-2000$ & Depleted \\
\hline Q76LX8 & ATS13_HUMAN & 63 & 2000 & 0.975 & 4.473 & 0.932 & $63-2000$ & Undepleted \\
\hline Q76LX8 & ATS13_HUMAN & 500 & 1000 & 0.956 & 4.141 & 0.999 & $500-1000$ & Depleted \\
\hline Q7KZ85 & SPT6H_HUMAN & 250 & 2000 & 0.61 & 7.159 & 0.84 & $250-2000$ & Undepleted \\
\hline Q7KZF4 & SND1_HUMAN & 63 & 2000 & 0.921 & 2.588 & 1.277 & $63-2000$ & Depleted \\
\hline Q7L1Q6 & BZW1_HUMAN & 63 & 2000 & 0.887 & 3.172 & 1.188 & $63-2000$ & Depleted \\
\hline Q7LDG7 & GRP2_HUMAN & $\begin{array}{c}\text { not } \\
\text { quantifiable }\end{array}$ & & & & & & Undepleted \\
\hline Q7Z3U7 & MON2_HUMAN & 125 & 1000 & 0.949 & 4.478 & 0.74 & $125-1000$ & Depleted \\
\hline Q7Z3Z3 & PIWL3_HUMAN & 500 & 2000 & 0.956 & 2.772 & 0.83 & $500-2000$ & Depleted \\
\hline Q7Z4W1 & DCXR_HUMAN & 250 & 2000 & 0.961 & 3.618 & 0.944 & $250-2000$ & Depleted \\
\hline Q7Z5P9 & MUC19_HUMAN & $\begin{array}{c}\text { not } \\
\text { quantifiable }\end{array}$ & & & & & & Depleted \\
\hline Q7Z7G0 & TARSH_HUMAN & 63 & 2000 & 0.961 & 1.883 & 0.939 & $63-2000$ & Depleted \\
\hline Q86UK5 & LBN_HUMAN & 63 & 2000 & 0.992 & 5.152 & 0.898 & $63-2000$ & Depleted \\
\hline Q86V81 & THOC4_HUMAN & 125 & 2000 & 0.977 & 5.398 & 0.844 & $125-2000$ & Undepleted \\
\hline Q86V81 & THOC4_HUMAN & 250 & 2000 & 0.619 & 5.547 & 0.518 & $250-2000$ & Depleted \\
\hline Q86VB7 & C163A_HUMAN & 1000 & 2000 & 0.967 & -1.538 & 1.532 & $1000-2000$ & Depleted \\
\hline Q86VP6 & CAND1_HUMAN & 63 & 2000 & 0.887 & 1.664 & 1.361 & $63-2000$ & Depleted \\
\hline Q86Y46 & K2C73_HUMAN & 63 & 2000 & 0.986 & 4.858 & 0.976 & $63-2000$ & Depleted \\
\hline Q8IVL1 & NAV2_HUMAN & 63 & 2000 & 0.917 & 4.137 & 0.772 & $63-2000$ & Depleted \\
\hline Q8IWV7 & UBR1_HUMAN & 500 & 500 & 0.966 & 4.638 & 0.885 & $N / A$ & Undepleted \\
\hline Q8IWV7 & UBR1_HUMAN & 63 & 1000 & 0.867 & 7.665 & 0.587 & $63-1000$ & Depleted \\
\hline Q8IXQ3 & CI040_HUMAN & 63 & 2000 & 0.905 & 3.901 & 0.875 & $63-2000$ & Depleted \\
\hline Q8IYS5 & OSCAR_HUMAN & $\begin{array}{c}\text { not } \\
\text { quantifiable }\end{array}$ & & & & & & Depleted \\
\hline Q8IZ83 & A16A1_HUMAN & 63 & 2000 & 0.922 & 5.596 & 0.923 & $63-2000$ & Depleted \\
\hline Q8N0V3 & RBFA_HUMAN & 63 & 2000 & 0.99 & 6.076 & 0.848 & $63-2000$ & Depleted \\
\hline Q8N392 & RHG18_HUMAN & $\begin{array}{c}\text { not } \\
\text { quantifiable }\end{array}$ & & & & & & Undepleted \\
\hline Q8N612 & F16A2_HUMAN & 500 & 500 & 0.754 & 4.26 & 0.771 & $\mathrm{~N} / \mathrm{A}$ & Depleted \\
\hline Q8NBJ4 & GOLM1_HUMAN & 250 & 2000 & 0.971 & 6.285 & 0.781 & $250-2000$ & Undepleted \\
\hline Q8NBP7 & PCSK9_HUMAN & $\begin{array}{c}\text { not } \\
\text { quantifiable }\end{array}$ & & & & & & Undepleted \\
\hline Q8NE71 & ABCF1_HUMAN & 250 & 2000 & 0.706 & 3.17 & 1.151 & $250-2000$ & Depleted \\
\hline Q8NHP8 & PLBL2_HUMAN & 250 & 2000 & 0.976 & 6.696 & 0.743 & $250-2000$ & Undepleted \\
\hline Q8NI22 & MCFD2_HUMAN & 250 & 2000 & 0.884 & 5.99 & 0.621 & $250-2000$ & Depleted \\
\hline Q8WXI9 & P66B_HUMAN & $\begin{array}{c}\text { not } \\
\text { quantifiable }\end{array}$ & & & & & & Undepleted \\
\hline Q8WZ74 & CTTB2_HUMAN & 500 & 2000 & 0.949 & -0.436 & 1.322 & $500-2000$ & Depleted \\
\hline Q92520 & FAM3C_HUMAN & 500 & 2000 & 0.881 & 5.146 & 0.677 & $500-2000$ & Depleted \\
\hline Q92619 & HMHA1_HUMAN & $\begin{array}{c}\text { not } \\
\text { quantifiable }\end{array}$ & & & & & & Undepleted \\
\hline Q92797 & SYMPK_HUMAN & 250 & 2000 & 0.925 & 4.617 & 0.777 & $250-2000$ & Depleted \\
\hline Q92820 & GGH_HUMAN & 63 & 2000 & 0.95 & -0.592 & 1.619 & $63-2000$ & Depleted \\
\hline
\end{tabular}




\begin{tabular}{|c|c|c|c|c|c|c|c|c|}
\hline Q92835 & SHIP1_HUMAN & 125 & 2000 & 0.99 & 5.12 & 0.778 & $125-2000$ & Undepleted \\
\hline Q92945 & FUBP2_HUMAN & $\begin{array}{c}\text { not } \\
\text { quantifiable }\end{array}$ & & & & & & Both \\
\hline Q92954 & PRG4_HUMAN & 250 & 2000 & 0.948 & -1.413 & 1.577 & $250-2000$ & Depleted \\
\hline Q93099 & HGD_HUMAN & 125 & 2000 & 0.953 & 7.275 & 0.717 & $125-2000$ & Undepleted \\
\hline Q96BZ4 & PLD4_HUMAN & 250 & 2000 & 0.914 & 5.824 & 0.726 & $250-2000$ & Depleted \\
\hline Q96IU4 & ABHEB_HUMAN & 125 & 2000 & 0.986 & 4.212 & 0.905 & $125-2000$ & Undepleted \\
\hline Q96IY4 & CBPB2_HUMAN & 1000 & 2000 & 0.697 & 1.484 & 0.96 & $1000-2000$ & Undepleted \\
\hline Q96IY4 & CBPB2_HUMAN & 63 & 2000 & 0.986 & 5.982 & 0.977 & $63-2000$ & Depleted \\
\hline Q96KN2 & CNDP1_HUMAN & 1000 & 2000 & 0.914 & -0.8 & 1.308 & $1000-2000$ & Undepleted \\
\hline Q96KN2 & CNDP1_HUMAN & 63 & 2000 & 0.964 & 3.187 & 1.195 & $63-2000$ & Depleted \\
\hline Q96PD5 & PGRP2_HUMAN & 250 & 2000 & 0.935 & 0.059 & 1.366 & $250-2000$ & Undepleted \\
\hline Q96PD5 & PGRP2_HUMAN & 63 & 2000 & 0.974 & 6.858 & 1.025 & $63-2000$ & Depleted \\
\hline Q96PK6 & RBM14_HUMAN & 250 & 2000 & 0.937 & 4.157 & 0.877 & $250-2000$ & Depleted \\
\hline Q99459 & CDC5L_HUMAN & $\begin{array}{c}\text { not } \\
\text { quantifiable }\end{array}$ & & & & & & Undepleted \\
\hline Q99460 & PSMD1_HUMAN & 125 & 2000 & 0.929 & 5.348 & 0.848 & $125-2000$ & Undepleted \\
\hline Q99460 & PSMD1_HUMAN & 250 & 2000 & 0.94 & 4.689 & 0.728 & $250-2000$ & Depleted \\
\hline Q99497 & PARK7_HUMAN & 125 & 2000 & 0.914 & 6.632 & 0.753 & $125-2000$ & Depleted \\
\hline Q99683 & M3K5_HUMAN & 125 & 2000 & 0.923 & 4.514 & 1 & $125-2000$ & Undepleted \\
\hline Q99683 & M3K5_HUMAN & 63 & 2000 & 0.915 & 6.292 & 0.897 & $63-2000$ & Depleted \\
\hline Q99729 & ROAA_HUMAN & 125 & 2000 & 0.92 & 4.794 & 1.017 & $125-2000$ & Depleted \\
\hline Q99784 & NOE1_HUMAN & 500 & 2000 & 0.93 & 2.416 & 0.853 & $500-2000$ & Depleted \\
\hline Q99829 & CPNE1_HUMAN & 250 & 2000 & 0.856 & 3.125 & 1.141 & $250-2000$ & Depleted \\
\hline Q99832 & TCPH_HUMAN & 63 & 2000 & 0.819 & 4.289 & 1.067 & $63-2000$ & Depleted \\
\hline Q99969 & RARR2_HUMAN & 500 & 2000 & 0.972 & 2.017 & 0.958 & $500-2000$ & Depleted \\
\hline Q9BQE5 & APOL2_HUMAN & 125 & 2000 & 0.889 & 3.018 & 0.977 & $125-2000$ & Depleted \\
\hline Q9BR76 & COR1B_HUMAN & 125 & 2000 & 0.985 & 3.958 & 0.852 & $125-2000$ & Undepleted \\
\hline Q9BTY2 & FUCO2_HUMAN & $\begin{array}{c}\text { not } \\
\text { quantifiable }\end{array}$ & & & & & & Depleted \\
\hline Q9BWP8 & COL11_HUMAN & $\begin{array}{c}\text { not } \\
\text { quantifiable }\end{array}$ & & & & & & Depleted \\
\hline Q9BXR6 & FHR5_HUMAN & 63 & 2000 & 0.955 & 3.601 & 1.037 & $63-2000$ & Depleted \\
\hline Q9BY67 & CADM1_HUMAN & 125 & 2000 & 0.954 & 5.653 & 0.817 & $125-2000$ & Undepleted \\
\hline Q9HOW9 & CK054_HUMAN & 125 & 2000 & 0.926 & 7.041 & 0.881 & $125-2000$ & Undepleted \\
\hline Q9HOW9 & CK054_HUMAN & 63 & 2000 & 0.905 & 4.286 & 0.838 & $63-2000$ & Depleted \\
\hline Q9H299 & SH3L3_HUMAN & $\begin{array}{c}\text { not } \\
\text { quantifiable }\end{array}$ & & & & & & Undepleted \\
\hline Q9H4A4 & AMPB_HUMAN & 250 & 2000 & 0.949 & 5.193 & 0.795 & $250-2000$ & Depleted \\
\hline Q9H4A9 & DPEP2_HUMAN & $\begin{array}{c}\text { not } \\
\text { quantifiable }\end{array}$ & & & & & & Depleted \\
\hline Q9H4M9 & EHD1_HUMAN & 125 & 2000 & 0.862 & 4.461 & 0.735 & $125-2000$ & Depleted \\
\hline Q9HAB8 & PPCS_HUMAN & 250 & 1000 & 0.837 & 2.94 & 1.032 & $250-1000$ & Depleted \\
\hline Q9HDC9 & APMAP_HUMAN & 1000 & 2000 & 0.485 & 3.739 & 0.682 & $1000-2000$ & Depleted \\
\hline Q9NNW7 & TRXR2_HUMAN & 125 & 2000 & 0.969 & 6.047 & 0.818 & $125-2000$ & Undepleted \\
\hline Q9NQ79 & CRAC1_HUMAN & 125 & 2000 & 0.967 & 2.007 & 1.116 & $125-2000$ & Depleted \\
\hline Q9NR12 & PDLI7_HUMAN & $\begin{array}{c}\text { not } \\
\text { quantifiable }\end{array}$ & & & & & & Undepleted \\
\hline
\end{tabular}




\begin{tabular}{|c|c|c|c|c|c|c|c|c|}
\hline Q9NTJ3 & SMC4_HUMAN & 125 & 2000 & 0.848 & 6.043 & 0.758 & $125-2000$ & Undepleted \\
\hline Q9NTX5 & ECHD1_HUMAN & $\begin{array}{c}\text { not } \\
\text { quantifiable }\end{array}$ & & & & & & Undepleted \\
\hline Q9NY33 & DPP3_HUMAN & 125 & 2000 & 0.874 & 6.405 & 0.87 & $125-2000$ & Undepleted \\
\hline Q9NY33 & DPP3_HUMAN & 125 & 2000 & 0.851 & 2.468 & 1.255 & $125-2000$ & Depleted \\
\hline Q9NYL2 & MLTK_HUMAN & 500 & 2000 & 0.912 & 5.214 & 0.725 & $500-2000$ & Depleted \\
\hline Q9NYU2 & UGGG1_HUMAN & 125 & 2000 & 0.982 & 6 & 0.787 & $125-2000$ & Undepleted \\
\hline Q9NZP8 & C1RL_HUMAN & 63 & 2000 & 0.964 & 4.592 & 1.058 & $63-2000$ & Depleted \\
\hline Q9P1Y5 & CAMP3_HUMAN & 125 & 500 & 0.836 & 5.394 & 0.737 & $125-500$ & Depleted \\
\hline Q9P260 & K1468_HUMAN & 63 & 2000 & 0.962 & 4.513 & 0.838 & $63-2000$ & Depleted \\
\hline Q9UBF2 & COPG2_HUMAN & 125 & 2000 & 0.836 & 4.148 & 0.925 & $125-2000$ & Depleted \\
\hline Q9UBR2 & CATZ_HUMAN & 500 & 2000 & 0.956 & 3.998 & 0.904 & $500-2000$ & Depleted \\
\hline Q9UGM5 & FETUB_HUMAN & 125 & 2000 & 0.958 & 2.732 & 0.925 & $125-2000$ & Undepleted \\
\hline Q9UGM5 & FETUB_HUMAN & 63 & 2000 & 0.958 & 4.637 & 1.086 & $63-2000$ & Depleted \\
\hline Q9UGT4 & SUSD2_HUMAN & $\begin{array}{c}\text { not } \\
\text { quantifiable }\end{array}$ & & & & & & Undepleted \\
\hline Q9UI26 & IPO11_HUMAN & 63 & 2000 & 0.926 & 4.8 & 0.791 & $63-2000$ & Depleted \\
\hline Q9UK55 & ZPI_HUMAN & 2000 & 2000 & 0.386 & -14.847 & 3.278 & $\mathrm{~N} / \mathrm{A}$ & Undepleted \\
\hline Q9UK55 & ZPI_HUMAN & 63 & 2000 & 0.945 & 4.333 & 1.05 & $63-2000$ & Depleted \\
\hline Q9UKX7 & NUP50_HUMAN & 63 & 2000 & 0.905 & 4.036 & 0.81 & $63-2000$ & Depleted \\
\hline Q9UNF1 & MAGD2_HUMAN & $\begin{array}{c}\text { not } \\
\text { quantifiable }\end{array}$ & & & & & & Undepleted \\
\hline Q9UNN8 & EPCR_HUMAN & 63 & 2000 & 0.947 & 1.923 & 1.199 & $63-2000$ & Depleted \\
\hline Q9UNU6 & CP8B1_HUMAN & 63 & 1000 & 0.946 & 3.415 & 0.887 & $63-1000$ & Depleted \\
\hline Q9UNW1 & MINP1_HUMAN & 500 & 2000 & 0.953 & 3.619 & 0.709 & $500-2000$ & Depleted \\
\hline Q9UNZ2 & NSF1C_HUMAN & 250 & 2000 & 0.851 & 5.172 & 0.667 & $250-2000$ & Depleted \\
\hline Q9UPT8 & ZC3H4_HUMAN & 500 & 2000 & 0.683 & 3.578 & 0.885 & $500-2000$ & Undepleted \\
\hline Q9UQ80 & PA2G4_HUMAN & 250 & 2000 & 0.831 & 5.161 & 0.716 & $250-2000$ & Depleted \\
\hline Q9Y230 & RUVB2_HUMAN & 63 & 2000 & 0.947 & 4.688 & 0.85 & $63-2000$ & Depleted \\
\hline Q9Y2E5 & MA2B2_HUMAN & 125 & 2000 & 0.981 & 4.557 & 0.883 & $125-2000$ & Undepleted \\
\hline Q9Y2G3 & AT11B_HUMAN & 125 & 2000 & 0.981 & 3.484 & 0.84 & $125-2000$ & Undepleted \\
\hline Q9Y2G3 & AT11B_HUMAN & 63 & 2000 & 0.955 & 5.672 & 0.846 & $63-2000$ & Depleted \\
\hline Q9Y2H5 & PKHA6_HUMAN & 2000 & 2000 & 0.815 & 4.475 & 0.485 & $\mathrm{~N} / \mathrm{A}$ & Undepleted \\
\hline Q9Y2H5 & PKHA6_HUMAN & 1000 & 2000 & 0.976 & 1.311 & 0.883 & $1000-2000$ & Depleted \\
\hline Q9Y2M0 & FAN1_HUMAN & 125 & 2000 & 0.883 & 1.716 & 0.955 & $125-2000$ & Undepleted \\
\hline Q9Y2M0 & FAN1_HUMAN & 63 & 2000 & 0.911 & 2.894 & 1.047 & $63-2000$ & Depleted \\
\hline Q9Y2X3 & NOP58_HUMAN & 500 & 2000 & 0.946 & 4.505 & 0.755 & $500-2000$ & Depleted \\
\hline Q9Y310 & RTCB_HUMAN & 125 & 2000 & 0.914 & 2.523 & 1.004 & $125-2000$ & Undepleted \\
\hline Q9Y3I0 & RTCB_HUMAN & 63 & 2000 & 0.902 & 2.605 & 1.166 & $63-2000$ & Depleted \\
\hline Q9Y446 & PKP3_HUMAN & $\begin{array}{c}\text { not } \\
\text { quantifiable }\end{array}$ & & & & & & Undepleted \\
\hline Q9Y490 & TLN1_HUMAN & 63 & 2000 & 0.808 & 1.707 & 1.568 & $63-2000$ & Depleted \\
\hline Q9Y4E8 & UBP15_HUMAN & 125 & 2000 & 0.881 & 3.378 & 0.938 & $125-2000$ & Depleted \\
\hline Q9Y4L1 & HYOU1_HUMAN & 63 & 2000 & 0.956 & 4.82 & 1.026 & $63-2000$ & Depleted \\
\hline Q9Y5K5 & UCHL5_HUMAN & 250 & 2000 & 0.935 & 5.173 & 0.782 & $250-2000$ & Depleted \\
\hline Q9Y5Y7 & LYVE1_HUMAN & 63 & 2000 & 0.907 & 2.22 & 1.151 & $63-2000$ & Depleted \\
\hline
\end{tabular}




\begin{tabular}{llccccccc} 
Q9Y608 & LRRF2_HUMAN & 250 & 2000 & 0.894 & 4.102 & 0.889 & $250-2000$ & Depleted \\
Q9Y617 & SERC_HUMAN & $\begin{array}{c}\text { not } \\
\text { quantifiable }\end{array}$ & & & & & Undepleted \\
Q9Y696 & CLIC4_HUMAN & 63 & 2000 & 0.906 & 5.539 & 0.79 & $63-2000$ & Depleted \\
Q9Y6R7 & FCGBP_HUMAN & 125 & 2000 & 0.954 & 6.288 & 0.838 & $125-2000$ & Undepleted \\
Q9Y6R7 & FCGBP_HUMAN & 63 & 2000 & 0.931 & 1.756 & 1.431 & $63-2000$ & Depleted \\
Q9Y6U3 & ADSV_HUMAN & $\begin{array}{c}\text { not } \\
\text { quantifiable } \\
\text { not } \\
\text { Q9Yantifiable }\end{array}$ & & & & & & Depleted \\
COL10_HUMAN & & & & & Depleted \\
\hline
\end{tabular}

